\title{
Diastereoselective $B\left(C_{6} F_{5}\right)_{3}$-Catalyzed Reductive Carbocyclization of Unsaturated Carbohydrates
}

\author{
Trandon A. Bender ${ }^{\dagger}$, Jennifer A. Dabrowski ${ }^{\ddagger}$, Hongyu Zhong ${ }^{\dagger}$, and Michel R. Gagné ${ }^{\dagger *}$ \\ 'Department of Chemistry, The University of North Carolina at Chapel Hill, Chapel Hill, NC 27599 \\ ${ }^{\ddagger}$ Department of Chemistry, Elon University, Elon, NC 27244
}

\section{Table of Contents:}

General Experimental

Preparation and Characterization of Substrates

Experimental Procedures

Representative preparation of deoxygentated substrates and silane deprotection of carbohydrate derivatives

Preparation of carbocylce 6 (Scheme 2)

Preparation of carbocycle 7 (Scheme 2)

Preparation of carbocycle 8 (Scheme 2)

Preparation of carbocycle 9 (Scheme 2)

Preparation of carbocycle 10 (Scheme 2)

Preparation of cyclic product 11 (Scheme 3)

Preparation and Kishi analysis of $\mathbf{S 1}$ to verify stereochemistry of $\mathbf{1 1}$ (Table 1)

Preparation of carbocycle 13 (Scheme 4)

Preparation of carbocycle 15 (Scheme 4)

Preparation of carbocycle $\mathbf{2 0}$ (Table 1)

Preparation of carbocycle 21 from 17 (Table 1)

Preparation of carbocycle $\mathbf{2 1}$ from $\mathbf{1 8}$ (Table 1)

Preparation of carbocycle 22 (Table 1)

Preparation of carbocycle derivative 23 (Figure 1)

Preparation of vinyl shift product $\mathbf{2 4}$ (Scheme 5) 
Preparation of vinyl shift product 26 (Scheme 5)

Preparation of carbocycle $\mathbf{2 0}$ from 27 (Scheme 6)

Preparation of cyclic product $\mathbf{2 8}$ (Scheme 6)

Preparation of vinyl shift product $\mathbf{2 4}$ from $\mathbf{2 8}$ (Scheme 6) 


\section{General Experimental}

General Methods: All catalytic reactions were performed in oven $\left(130^{\circ} \mathrm{C}\right)$ and/or flamedried glassware in a nitrogen-filled glovebox unless otherwise specified. All reactions were performed at ambient temperature $\left(25^{\circ} \mathrm{C}, \mathrm{RT}\right)$ unless otherwise specified. "Dowex ${ }^{\circledR}$ resin" refers to Dowex ${ }^{\circledR} 50 \mathrm{~W}-\mathrm{X} 8$, a strongly acidic cation exchange resin distributed by Baker Chemical Company. Before use, the orange Dowex ${ }^{\circledR}$ resin beads were washed five times with methanol and five times with methylene chloride and then allowed to dry under nitrogen flow. All workup procedures were performed under air with reagent grade reagents unless otherwise specified. Alumina refers to Brockmann activity I neutral alumina gel (Aldrich) that was treated with $10 \%$ deionzed water as previously disclosed. ${ }^{1}$ Column chromatography was performed using SilaFlash P60 40-63 $\mu \mathrm{m}$ (230-400 mesh) or the pretreated alumina gel described above. Thin layer chromatography (TLC) was performed on SiliCycle Silica Gel 60 F254 plates and was visualized with UV light, cerium ammonium molybdate (CAM) stain, or potassium permanganate $\left(\mathrm{KMnO}_{4}\right)$ stain.

All NMR spectra were recorded either on a Bruker Avance $600 \mathrm{MHz}, 500 \mathrm{MHz}$, or 400 $\mathrm{MHz}$ spectrometer at standard temperature and pressure. All deuterated solvents were used as received from Cambridge Isotope Laboratories, Inc. The residual solvent protons $\left({ }^{1} \mathrm{H}\right)$ or the solvent carbons $\left({ }^{13} \mathrm{C}\right)$ were used as internal references. ${ }^{2}$ The following abbreviations are used in reporting NMR data: $s$, singlet; $d$, doublet; $t$, triplet; q, quartet; quint, quintet; sept, septet; dd, doublet of doublets; dt, doublet of triplets; dq, doublet of quartets; td, triplet of doublets; tt, triplet of triplets; quint d, quintet of doublets; ddd, doublet of doublet of doublets; and m, multiplet. Where necessary, 2D COSY, HSQC, HMBC, and APT data were used for peak assignment. High resolution mass spectra were obtained on either an Agilent Accurate LC-TOF Mass Spectrometer (Agilent Series 6220) operating in positive ion mode with an electrospray ionization source (fragmentor $=175 \mathrm{~V}$ ) or with a hybrid LTQ FT (ICR 7T) (ThermoFisher, Bremen, Germany) mass spectrometer where samples were introduced via a micro-electrospray source at a flow rate of $3 \mu \mathrm{L} / \mathrm{min}$. The data were analyzed using either Agilent MassHunter Workstation Software, Qualitative Analysis (V. B.02.00) or Xcalibur (ThermoFisher, Breman, Germany). Specific rotations were obtained on a Jasco DIP-1000 digital polarimeter equipped with a sodium lamp at STP utilizing a $3.5 \times 100 \mathrm{~mm}$ cell. A NesLab cryobath CB- 80 was utilized for reduced temperature reactions where temperature was verified by low-temperature thermometer.

Chemicals: D-galactose, D-mannose, D-glucose, D-ribose were purchased from SigmaAldrich and used as received. $\mathrm{Me}_{2} \mathrm{EtSiH}_{2} \mathrm{Et}_{3} \mathrm{SiH}$, and $\mathrm{Et}_{3} \mathrm{SiD}(97 \% \mathrm{D})$ were purchased from Sigma-Aldrich and stored over pre-activated molecular sieves in the glovebox. $\mathrm{Ph}_{3} \mathrm{SiH}$ was purchased from Sigma-Aldrich and used as received. Dioxane (anhydrous, 99\%), pyridine (anhydrous, 99\%), benzyltriphenylphosphonium chloride, potassium tertbutoxide, dichloromethane, triethylamine, 3-bromo-1-phenyl-1-propene (cinnamyl 
bromide), 3,3-dimethylallylbromide, and copper sulfate were purchased from SigmaAldrich and used as received. Methanol was purchased from Fisher and used as received. $\mathrm{CH}_{2} \mathrm{Cl}_{2}$ (used for reactions) and toluene (for trituration) was passed through an alumina column in a solvent purification system prior to use. Tris(pentafluorophenyl)borane $\left(\mathrm{B}\left(\mathrm{C}_{6} \mathrm{~F}_{5}\right)_{3}\right)$ and Trityl tetrakis(pentafluorophenyl)borate was purchased from Strem and used as received. It is worth noting that the source of $\mathrm{B}\left(\mathrm{C}_{6} \mathrm{~F}_{5}\right)_{3}$ is important to this chemistry and best results were found when using the source above. Alkenyl carbohyrdates were prepared using a previously described Wittig olefination protocol and their identity was verified by comparison with published data prior to protection.

All alkenyl substrates were prepared following a modified protocol from a previous publication. ${ }^{3}$ A flame-dried $500 \mathrm{~mL}$ flask with a magnetic stir bar was placed under a flow of nitrogen. Benzyltriphenylphosphonium chloride (11.6 g, $30 \mathrm{mmol})$ followed by potassium tert-butoxide was added to the flask. A reflux condenser was attached and anhydrous dioxane $(120 \mathrm{~mL})$ was added. The reaction was allowed to stirred for 30 minutes at $25{ }^{\circ} \mathrm{C}$ and then placed into an $80{ }^{\circ} \mathrm{C}$ oil bath to stir for an additional 30 minutes. The sugar was then added under positive nitrogen pressure and the reaction was allowed to reflux for $2 \mathrm{~h}$ (the reaction went from dark red to yellow over the reaction time). Water $(10 \mathrm{~mL})$ was added and the reaction was concentrated under vacuum to yield a sticky yellow material. For preparation of $\mathbf{1 6}$ the $E$-olefin was isolated by crystallization from warm methanol. The other products were isolated by silica gel chromatography ( $30 \times 5 \mathrm{~cm}$ column) using gradient elution (20:1 to $15: 1$ to $10: 1$ to $7: 1$ $\mathrm{CH}_{2} \mathrm{Cl}_{2}: \mathrm{MeOH}$ ) to afford the unprotected alkenyl carbohydrates.

Diene substrates were prepared by analogy to the above procedure. The phosphonium salts were prepared prior to use as follows. A flame-dried $250 \mathrm{~mL}$ flask with a magnetic sitr bar was placed under a flow of nitrogen. Triphenylphosphine (11.5 g, $44 \mathrm{mmol})$ was added to the flask followed by toluene $(100 \mathrm{~mL})$ by syringe. Cinnamyl bromide $(7.9 \mathrm{~g}, 40$ mmol) was then added by syringe under positive nitrogen. The flask was attached to a reflux condenser and allowed to reflux for $12 \mathrm{~h}$. The white solid that formed during the reaction was collected by vacuum filtration, rinsed with cold diethyl ether, placed under high-vacuum for 3 hours, and used as the phosphonium bromide salt under the reaction conditions described above. Prenylphosphonium salt was prepared in an analogous manner by combining triphenylphosphine $(8.6 \mathrm{~g}, 33 \mathrm{mmol})$ and prenylbromide $(3.5 \mathrm{~mL}$, $30 \mathrm{mmol}$ ) with the same reaction and isolation protocol given above.

\section{Preparation and Characterization of Substrates}

All protected substrates were prepared by a modification of an existing protocol. ${ }^{4}$ The substrate (5.0 mmol, 1.0 equiv.) was added to a $20 \mathrm{~mL}$ scintillation vial equipped with a magnetic stir bar. Nitrogen was then purged through the flask $(5 \mathrm{~min})$ by fitting a septum with a vent needle to the vial. This was followed by addition of $\mathrm{CH}_{2} \mathrm{Cl}_{2}(5.0 \mathrm{~mL})$ by 
syringe. To this solution was added pyridine $(6.5 \mathrm{mmol}, 1.1$ equiv. per $-\mathrm{OH}$ group plus 1.0 equiv.) followed by the dropwise addition of chlorotrialkylsilane (5.5 equiv., 1.1 equiv. per $-\mathrm{OH}$ group). The vial was capped and allowed to stir at $25{ }^{\circ} \mathrm{C}$ for $12 \mathrm{~h}$. After the reaction time, deionized water was added $(5.0 \mathrm{~mL})$ and the entire reaction mixture was transferred to a separatory funnel, the reaction vessel was rinsed with diethyl ether $(3 \times 5 \mathrm{~mL})$ and added to the separatory funnel. An additional $15 \mathrm{~mL}$ of diethyl ether was added and the layers separated. The aqueous layer was subsequently washed with diethyl ether $(2 \times 20 \mathrm{~mL})$ and all organic layers then combined. In the cases of a volatile substrate, the organic layers were washed with a $10 \%(\mathrm{w} / \mathrm{w})$ of $\mathrm{CuSO}_{4}$ solution until the color did not visibly change. The organic layers were washed with water $(3 \times 20 \mathrm{~mL})$, brine ( $3 \times 20 \mathrm{~mL})$, and then dried over sodium sulfate. The solution was filtered over a cotton plug and the organic layer was concentrated down to yield the final product as either a white solid or a colorless oil. Trituration with toluene was preformed on nonvolatile materials to remove pyridine.

To remove residual pyridine salts, the substrate was passed over an alumina column (neutral, 10\% $\mathrm{H}_{2} \mathrm{O} ; 3 \times 1$ in) using gradient elution (100\% to 95:5 hexanes/diethyl ether). Fractions containing product were collected and concentrated. The residue was then

placed under high vacuum for a minimum of $12 \mathrm{~h}$ prior to bringing into the glovebox for use, where they were stored in scintillation vials in a nitrogen filled glovebox at $25^{\circ} \mathrm{C}$ and used as prepared.

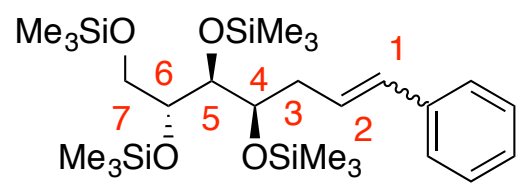

1

Me $_{3}$ Si-styryl-2-deoxyglucose (1): Prepared and used as a 86:14 E/Z mixture. ${ }^{1} \mathrm{H}$ NMR $\left(600 \mathrm{MHz}, \mathrm{CDCl}_{3}\right): \delta 7.35-7.19(\mathrm{~m}, 8 \mathrm{H}, \mathrm{Ph}, E / Z), 7.24-7.17$ (m, 2H, Ph, E/Z), 6.49 (d, $J$ $=11.8 \mathrm{~Hz}, 1 \mathrm{H}, Z), 6.42(\mathrm{~d}, J=15.9 \mathrm{~Hz}, 1 \mathrm{H}, E), 6.22(\mathrm{ddd}, J=14.4,8.4 \mathrm{~Hz}, 1 \mathrm{H}, E), 5.73$ (ddd, $J=14.4,7.2 \mathrm{~Hz}, 1 \mathrm{H}, Z), 3.87-3.81(\mathrm{~m}, 3 \mathrm{H}, Z / E), 3.75(\mathrm{dd}, J=10.5,3.7 \mathrm{~Hz}, 1 \mathrm{H}, E)$, 3.73-3.67 (m, 2H, Z), 3.64-3.56 (m, 2H, Z/E), 3.48 (dd, J=7.2, 3.6 Hz, 1H, E), 3.43 (dd, $J=6.6,3.6 \mathrm{~Hz}, 1 \mathrm{H}, Z), 2.60-2.56(\mathrm{~m}, 2 \mathrm{H}, Z), 2.54-2.50(\mathrm{~m}, 1 \mathrm{H}, E), 2.39-2.34(\mathrm{~m}, 1 \mathrm{H}$, E), $0.14\left(\mathrm{~s}, 9 \mathrm{H},-\mathrm{Si}\left(\mathrm{CH}_{3}\right)_{3}\right), 0.12\left(\mathrm{~s}, 18 \mathrm{H},-\mathrm{Si}\left(\mathrm{CH}_{3}\right)_{3}\right), 0.10\left(\mathrm{~s}, 9 \mathrm{H},-\mathrm{Si}\left(\mathrm{CH}_{3}\right)_{3}\right), 0.083$ (s, 9H, $\left.-\mathrm{Si}\left(\mathrm{CH}_{3}\right)_{3}\right), 0.071\left(\mathrm{~s}, 9 \mathrm{H},-\mathrm{Si}\left(\mathrm{CH}_{3}\right)_{3}\right) .{ }^{13} \mathrm{C}\left\{{ }^{1} \mathrm{H}\right\} \mathrm{NMR}\left(151 \mathrm{MHz}, \mathrm{CDCl}_{3}\right): \delta 137.9(\mathrm{~s})$, 137.7 (s), 132.1 (s), 130.4 (s), 129.4 (s), 129.0 (s), 128.6 (s), 128.3 (s), 127.6 (s), 127.1 (s), 126.7 (s), 126.1 (s), 78.7 (s), 78.2 (s), 74.3 (s), 74.27 (s), 74.0 (s), 73.9 (s), 64.0 (s), 63.9 (s), 37.6 (s), 33.0 (s), 0.84, 0.81, 0.66, 0.54, 0.50, -0.51, -0.55 (each a s, $\left.-\mathrm{Si}\left(\mathrm{CH}_{3}\right)_{3}\right)$. (*Note: one $Z / E$-TMS group is overlapping.) 


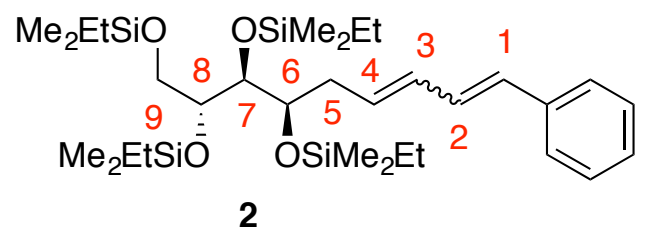

$\mathbf{M e}_{2}$ EtSi-styryl-2-deoxyglucose diene (2): Prepared and used as a 65:35 E,E/E,Z mixture. ${ }^{1} \mathrm{H}$ NMR (600 MHz, $\mathrm{CDCl}_{3}$ ): $\delta$ 7.45-7.36 (m, 4H, Ph, E,E/E,Z), 7.35-7.28 (m, 4H, Ph, E,E/E,Z), 7.23-7.19 (m, 2H, Ph, E,E/E,Z), 7.05 (ddd, $J=15.5 \mathrm{~Hz}, 11.1 \mathrm{~Hz}, 1.2$ $\mathrm{Hz}, 1 \mathrm{H}, E, Z), 6.76$ (ddd, $J=15.7,10.4,0.8 \mathrm{~Hz}, 1 \mathrm{H}, E, E), 6.53$ (d, $J=15.6 \mathrm{~Hz}, 1 \mathrm{H}, E, Z)$, $6.45(\mathrm{~d}, J=15.7 \mathrm{~Hz}, 1 \mathrm{H}, E, E), 6.27-6.13(\mathrm{~m}, 2 \mathrm{H}, E, E / E, Z), 5.83(\mathrm{~m}, 1 \mathrm{H}, E, E), 5.59$ (dt, $J$ $=10.7 \mathrm{~Hz}, 7.7 \mathrm{~Hz}, 1 \mathrm{H}, E, Z), 3.85-3.80(\mathrm{~m}, 2 \mathrm{H}, E, E / E, Z), 3.80-3.68(\mathrm{~m}, 4 \mathrm{H}, E, E / E, Z)$, $3.62(\mathrm{dd}, J=5.5 \mathrm{~Hz}, 2.6 \mathrm{~Hz}, 1 \mathrm{H}, E, Z), 3.60(\mathrm{dd}, J=5.7 \mathrm{~Hz}, 2.5 \mathrm{~Hz}, 1 \mathrm{H}, E, E), 3.51-3.42$ (m, 2H, E,E/E,Z), 2.57-2.49 (m, 2H, E,Z), 2.48-2.43 (m, 1H, E,E), 2.33-2.26 (m, 1H, $E, E), 0.99-0.88\left(\mathrm{~m}, 24 \mathrm{H},-\mathrm{Si}\left(\mathrm{CH}_{3}\right)_{2}\left(\mathrm{CH}_{2} \mathrm{CH}_{3}\right)\right), 0.64-0.53\left(\mathrm{~m}, 16 \mathrm{H},-\mathrm{Si}\left(\mathrm{CH}_{3}\right)_{2}\left(\mathrm{CH}_{2} \mathrm{CH}_{3}\right)\right)$, 0.11-0.06 (m, 48H, - $\left.\mathrm{Si}\left(\mathrm{CH}_{3}\right)_{2}\left(\mathrm{CH}_{2} \mathrm{CH}_{3}\right)\right)$. (*Note: E,E/E, $\mathrm{Z} \quad \mathrm{Si}\left(\mathrm{CH}_{3}\right)_{2}\left(\mathrm{CH}_{2} \mathrm{CH}_{3}\right)$ are overlapping). ${ }^{13} \mathrm{C}\left\{{ }^{1} \mathrm{H}\right\}$ NMR $\left(151 \mathrm{MHz}, \mathrm{CDCl}_{3}\right): \delta 137.6(\mathrm{~s}), 137.6(\mathrm{~s}), 132.6(\mathrm{~s}), 132.4$ (s), $132.3(\mathrm{~s}), 130.3(\mathrm{~s}), 130.1(\mathrm{~s}), 129.6(\mathrm{~s}), 129.3(\mathrm{~s}), 128.5(\mathrm{~s}), 128.5(\mathrm{~s}), 127.3(\mathrm{~s})$, $127.1(\mathrm{~s}), 126.3(\mathrm{~s}), 126.2(\mathrm{~s}), 124.6(\mathrm{~s}), 78.7(\mathrm{~s}), 78.4(\mathrm{~s}), 74.6(\mathrm{~s}), 74.3(\mathrm{~s}), 74.1(\mathrm{~s}), 74.0$ (s), $64.2(\mathrm{~s}), 64.0(\mathrm{~s}), 37.3(\mathrm{~s}), 32.2(\mathrm{~s}), 8.9,8.84,8.83,8.7,8.0,7.9,7.0,6.96,6.95,6.93$, $6.9,6.8,-1.5,-1.6,-1.62,-1.64,-1.69,-1.7,-1.8,-1.9,-2.7,-2.74,-2.76,-2.8$ (each a s, $\mathrm{Si}\left(\mathrm{CH}_{3}\right)_{2}\left(\mathrm{CH}_{2} \mathrm{CH}_{3}\right)$, $-\mathrm{Si}\left(\mathrm{CH}_{3}\right)_{2}\left(\mathrm{CH}_{2} \mathrm{CH}_{3}\right)$ or $\left.-\mathrm{Si}\left(\mathrm{CH}_{3}\right)_{2}\left(\mathrm{CH}_{2} \mathrm{CH}_{3}\right)\right)$. ( Note: Overlapping in silane region).

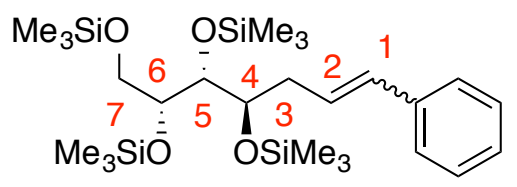

3

$\mathrm{Me}_{3}$ Si-styryl-2-deoxygalactose (3): Prepared and used as a 77:23 E/Z mixture. ${ }^{1} \mathrm{H}$ NMR (400 MHz, $\mathrm{CDCl}_{3}$ ): $\delta 7.41-7.28$ (m, 8H, Ph, E/Z), 7.24-7.17 (m, 2H, Ph, E/Z), 6.51 (dd, $J$ $=11.7,1.8 \mathrm{~Hz}, 1 \mathrm{H}, Z), 6.41(\mathrm{~d}, J=15.9 \mathrm{~Hz}, 1 \mathrm{H}, E), 6.26(\mathrm{dt}, J=15.8,7.2 \mathrm{~Hz}, 1 \mathrm{H}, E)$, 5.74 (ddd, $J=11.7,8.0 \mathrm{~Hz}, 6.8 \mathrm{~Hz}, 1 \mathrm{H}, Z), 3.94(\mathrm{t}, J=2.9 \mathrm{~Hz}, 1 \mathrm{H}, Z), 3.91$ (ddd, $J=7.4$, $4.3,2.6 \mathrm{~Hz}, 1 \mathrm{H}, E), 3.76-3.67(\mathrm{~m}, 4 \mathrm{H}, E / Z), 3.66-3.63(\mathrm{~m}, 1 \mathrm{H}, E), 3.62(\mathrm{~d}, J=4.5 \mathrm{~Hz}$, $1 \mathrm{H}, Z), 3.58(\mathrm{dd}, J=9.9 \mathrm{~Hz}, 5.2 \mathrm{~Hz}, 1 \mathrm{H}, E), 3.55-3.48(\mathrm{~m}, 1 \mathrm{H}, Z), 2.82-2.67(\mathrm{~m}, 1 \mathrm{H}, Z)$, 2.50-2.40 (m, 3H, E/Z), 0.18 (s, 9H, $\left.-\mathrm{Si}\left(\mathrm{CH}_{3}\right)_{3}\right), 0.17$ (s, 9H, $\left.-\mathrm{Si}\left(\mathrm{CH}_{3}\right)_{3}\right), 0.14$ (s, 9H, $\left.\mathrm{Si}\left(\mathrm{CH}_{3}\right)_{3}\right), 0.14$ (s, 9H, - $\left.\mathrm{Si}\left(\mathrm{CH}_{3}\right)_{3}\right), 0.13$ (s, 9H, - $\left.\mathrm{Si}\left(\mathrm{CH}_{3}\right)_{3}\right), 0.12$ (s, 9H, $\left.-\mathrm{Si}\left(\mathrm{CH}_{3}\right)_{3}\right), 0.12$ (s, 9H, - $\left.\mathrm{Si}\left(\mathrm{CH}_{3}\right)_{3}\right)$. (*Note: one $\mathrm{Z} / \mathrm{E}$-TMS group is overlapping.) ${ }^{13} \mathrm{C} \mathrm{NMR}(101 \mathrm{MHz}$, $\left.\mathrm{CDCl}_{3}\right): \delta 137.9(\mathrm{~s}), 137.7(\mathrm{~s}), 131.5(\mathrm{~s}), 130.4(\mathrm{~s}), 130.1(\mathrm{~s}), 128.8(\mathrm{~s}), 128.7(\mathrm{~s}), 128.4$ (s), $128.0(\mathrm{~s}), 126.7(\mathrm{~s}), 126.4(\mathrm{~s}), 125.9(\mathrm{~s}), 78.1(\mathrm{~s}), 77.9(\mathrm{~s}), 75.5(\mathrm{~s}), 75.4(\mathrm{~s}), 73.7(\mathrm{~s})$, $64.1(\mathrm{~s}), 64.1(\mathrm{~s}), 36.4(\mathrm{~s}), 31.8(\mathrm{~s}), 0.7,0.68,0.6,0.57,0.5,-0.46,-0.5$ (each a s, - 
$\left.\mathrm{Si}\left(\mathrm{CH}_{3}\right)_{3}\right)$. (*Note: one $Z / E$-TMS group is overlapping and one $Z / E \mathrm{C}-\mathrm{O}$ carbon is overlapping.)

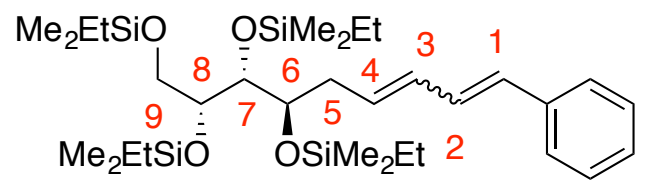

4

$\mathbf{M e}_{2}$ EtSi-styryl-2-deoxygalactose diene (4): Prepared and used as a $76: 34 E, E / E, Z$ mixture. ${ }^{1} \mathrm{H} \mathrm{NMR}\left(600 \mathrm{MHz}, \mathrm{CDCl}_{3}\right): \delta 7.45-7.36(\mathrm{~m}, 4 \mathrm{H}, \mathrm{Ph}, E, E / E, Z), 7.36-7.29$ (m, $4 \mathrm{H}, \mathrm{Ph}, E, E / E, Z), 7.24-7.17$ (m, 2H, Ph, E,E/E,Z), 7.10 (ddt, $J=15.5,11.1,1.2 \mathrm{~Hz}, 1 \mathrm{H}$, $E, Z), 6.76(\mathrm{dd}, J=15.7,10.4 \mathrm{~Hz}, 1 \mathrm{H}, E, E), 6.53(\mathrm{~d}, J=15.6 \mathrm{~Hz}, 1 \mathrm{H}, E, Z), 6.45$ (d, $J=$ $15.7 \mathrm{~Hz}, 1 \mathrm{H}, E, E), 6.31-6.13(\mathrm{~m}, 2 \mathrm{H}, E, E / E, Z), 5.83(\mathrm{dt}, J=14.9,7.3 \mathrm{~Hz}, 1 \mathrm{H}, E, E), 5.59$ (dt, $J=10.7,7.7 \mathrm{~Hz}, 1 \mathrm{H}, E, Z), 3.91-3.81(\mathrm{~m}, 2 \mathrm{H}, E, E / E, Z), 3.76-3.73(\mathrm{~m}, 1 \mathrm{H}, E, Z)$, 3.72-3.63 (m, 5H, E,E/E,Z), 3.61-3.53 (m, 2H, E,E/E,Z), 2.66-2.56 (m, 1H, E,Z), 2.46$2.30(\mathrm{~m}, 3 \mathrm{H}, E, E / E, Z), 1.03-0.87\left(\mathrm{~m}, 24 \mathrm{H},-\mathrm{Si}\left(\mathrm{CH}_{3}\right)_{2}\left(\mathrm{CH}_{2} \mathrm{CH}_{3}\right)\right), 0.70-0.51(\mathrm{~m}, 16 \mathrm{H},-$ $\left.\mathrm{Si}\left(\mathrm{CH}_{3}\right)_{2}\left(\mathrm{CH}_{2} \mathrm{CH}_{3}\right)\right), \quad 0.18-0.06 \quad\left(\mathrm{~m}, \quad 48 \mathrm{H}, \quad-\mathrm{Si}\left(\mathrm{CH}_{3}\right)_{2}\left(\mathrm{CH}_{2} \mathrm{CH}_{3}\right)\right)$. (*Note: $E, E / E, Z$ $\mathrm{Si}\left(\mathrm{CH}_{3}\right)_{2}\left(\mathrm{CH}_{2} \mathrm{CH}_{3}\right)$ are overlapping). ${ }^{13} \mathrm{C}\left\{{ }^{1} \mathrm{H}\right\}$ NMR $\left(151 \mathrm{MHz}, \mathrm{CDCl}_{3}\right): \delta 137.6(\mathrm{~s})$, $137.6(\mathrm{~s}), 132.6$ (s), 132.4 (s), 132.3 (s), 130.3 (s), 130.1 (s), 129.6 (s), 129.3 (s), 128.5 (s), $128.5(\mathrm{~s}), 127.3(\mathrm{~s}), 127.1(\mathrm{~s}), 126.3(\mathrm{~s}), 126.2(\mathrm{~s}), 124.6(\mathrm{~s}), 78.1(\mathrm{~s}), 78.0(\mathrm{~s}), 76.0$ (s), $75.9(\mathrm{~s}), 73.8(\mathrm{~s}), 73.7(\mathrm{~s}), 64.1(\mathrm{~s}), 36.4(\mathrm{~s}), 31.5(\mathrm{~s}), 8.8,8.78,8.76,8.74,8.7,8.0$, 7.9, 7.0, 6.96, 6.94, 6.9, 6.8, -1.66, -1.69, -1.7, -1.74, -1.77, -1.81, 1.83, -1.85, -1.90 -2.7, -2.7, (each a s, $-\mathrm{Si}\left(\mathrm{CH}_{3}\right)_{2}\left(\mathrm{CH}_{2} \mathrm{CH}_{3}\right),-\mathrm{Si}\left(\mathrm{CH}_{3}\right)_{2}\left(\mathrm{CH}_{2} \mathrm{CH}_{3}\right)$ or $\left.-\mathrm{Si}\left(\mathrm{CH}_{3}\right)_{2}\left(\mathrm{CH}_{2} \mathrm{CH}_{3}\right)\right)$. (*Note: Overlap of $1 \mathrm{C}-\mathrm{O}$ bond and overlapping in silane region).

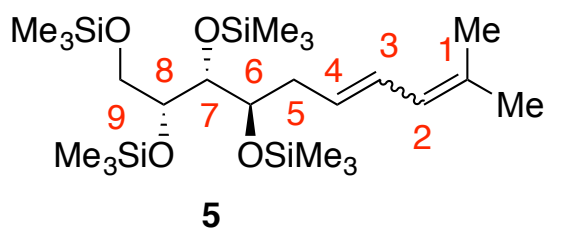

$\mathrm{Me}_{3} \mathrm{Si}$-2-deoxygalactose prenyldiene (5): Prepared and used as a 68:32 E,E/E,Z mixture. ${ }^{1} \mathrm{H}$ NMR $\left(600 \mathrm{MHz}, \mathrm{CDCl}_{3}\right): \delta 6.28-6.17(\mathrm{~m}, 2 \mathrm{H}, E, E / E, Z), 6.08$ (ddt, $J=11.3$, $2.8,1.4 \mathrm{~Hz}, 1 \mathrm{H}, E, Z), 5.78(\mathrm{ddt}, J=10.8,2.4,1.3 \mathrm{~Hz}, 1 \mathrm{H}, E, E), 5.53(\mathrm{dt}, J=14.9,7.4 \mathrm{~Hz}$, $1 \mathrm{H}, E, E), 5.37$ (dt, $J=11.0,7.5 \mathrm{~Hz}, 1 \mathrm{H}, E, Z), 3.82-3.74$ (m, 2H, $E, E / E, Z), 3.69-3.59$ (m, $6 \mathrm{H}, E, E / E, Z), 3.55-3.50(\mathrm{~m}, 2 \mathrm{H}, E, E / E, Z), 2.45-2.33(\mathrm{~m}, 2 \mathrm{H}, E, Z), 2.31-2.27(\mathrm{~m}, 2 \mathrm{H}$, $E, E), 1.79$ (s, 3H, E,Z), 1.75 (s, 3H, E,E), 1.74 (s, 3H, E,Z), 1.73 (s, 3H, E,E), 0.14 (s, $18 \mathrm{H}, E, E / E, Z), 0.13$ (s, 18H, E,E/E,Z), 0.125 (s, 9H, E,Z), 0.11 (s, 18H, E,E/E,Z), 0.08 (s, 9H, E,E). ${ }^{13} \mathrm{C}\left\{{ }^{1} \mathrm{H}\right\}$ NMR $\left(151 \mathrm{MHz}, \mathrm{CDCl}_{3}\right): \delta 134.6(\mathrm{~s}), 132.7(\mathrm{~s}), 129.2(\mathrm{~s}), 128.6$ (s), $126.6(\mathrm{~s}), 126.0(\mathrm{~s}), 125.1(\mathrm{~s}), 121.0(\mathrm{~s}), 77.81(\mathrm{~s}), 77.8(\mathrm{~s}), 75.4(\mathrm{~s}), 75.3(\mathrm{~s}), 73.8(\mathrm{~s})$, 
$73.6(\mathrm{~s}), 64.1(\mathrm{~s}), 64.0(\mathrm{~s}), 36.2(\mathrm{~s}), 30.8(\mathrm{~s}), 26.3(\mathrm{~s}), 25.9$ (s), 18.2 (s), $18.0(\mathrm{~s}), 0.73$, $0.71,0.69,0.6,0.5,-0.48$ each a s, $-\mathrm{Si}\left(\mathrm{CH}_{3}\right)_{3}$. (*Note: two sets of silane peaks overlap in the carbon NMR).

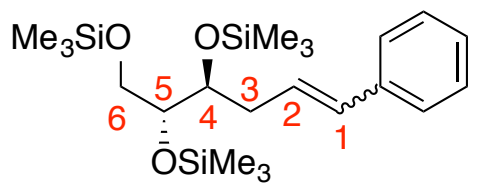

12

$\mathrm{Me}_{3}$ Si-styryl-2-deoxyribose (12): Prepared and used as a 85:15 E/Z mixture. ${ }^{1} \mathrm{H}$ NMR (400 MHz, $\mathrm{CDCl}_{3}$ ): $\delta$ 7.36-7.33 (m, 4H, Ph, E/Z), 7.33-7.28 (m, 4H, Ph, E/Z), 7.23-7.17 (m, 2H, Ph, E/Z), 6.52 (d, $J=11.7 \mathrm{~Hz}, 1 \mathrm{H}, Z), 6.43$ (d, $J=15.9 \mathrm{~Hz}, 1 \mathrm{H}, E), 6.24$ (ddd, $J$ $=15.8,7.8,6.8 \mathrm{~Hz}, 1 \mathrm{H}, E), 5.76(\mathrm{dt}, J=11.7,7.4 \mathrm{~Hz}, 1 \mathrm{H}, Z), 3.83-3.78(\mathrm{~m}, 2 \mathrm{H}, E / Z)$, 3.69 (dd, $J=10.3,3.7 \mathrm{~Hz}, 1 \mathrm{H}, E), 3.66-3.59$ (m, $3 \mathrm{H}, E / Z), 3.54$ (dd, $J=10.3,6.0 \mathrm{~Hz}$, $1 \mathrm{H}, E), 3.50(\mathrm{dd}, J=10.2,6.0 \mathrm{~Hz}, 1 \mathrm{H}, Z), 2.58-2.54(\mathrm{~m}, 2 \mathrm{H}, Z), 2.52-2.46(\mathrm{~m}, 1 \mathrm{H}, E)$, 2.41-2.32 (m, 1H, E), $0.15\left(\mathrm{~s}, 9 \mathrm{H},-\mathrm{Si}\left(\mathrm{CH}_{3}\right)_{3}\right), 0.13\left(\mathrm{~s}, 9 \mathrm{H},-\mathrm{Si}\left(\mathrm{CH}_{3}\right)_{3}\right), 0.12$ (s, 9H, $\left.\mathrm{Si}\left(\mathrm{CH}_{3}\right)_{3}\right), 0.10$ (s, 9H, - $\left.\mathrm{Si}\left(\mathrm{CH}_{3}\right)_{3}\right), 0.10$ (s, 2H, $\left.-\mathrm{Si}\left(\mathrm{CH}_{3}\right)_{3}\right), 0.08$ (s, 9H, $\left.-\mathrm{Si}\left(\mathrm{CH}_{3}\right)_{3}\right)$. ${ }^{13} \mathrm{C}\left\{{ }^{1} \mathrm{H}\right\}$ NMR $\left(151 \mathrm{MHz}, \mathrm{CDCl}_{3}\right):{ }^{13} \mathrm{C}\left\{{ }^{1} \mathrm{H}\right\}$ NMR $\left(151 \mathrm{MHz}, \mathrm{CDCl}_{3}\right): \delta 137.7(\mathrm{~s}), 137.5$ (s), $132.1(\mathrm{~s}), 130.4(\mathrm{~s}), 129.3(\mathrm{~s}), 128.8(\mathrm{~s}), 128.5$ (s), $128.0(\mathrm{~s}), 127.5$ (s), $126.9(\mathrm{~s})$, $126.5(\mathrm{~s}), 125.9(\mathrm{~s}), 76.6(\mathrm{~s}), 76.57(\mathrm{~s}), 73.6(\mathrm{~s}), 73.5(\mathrm{~s}), 64.0(\mathrm{~s}), 63.9(\mathrm{~s}), 37.1(\mathrm{~s}), 32.3$ (s), $0.54,0.5,0.46,0.4,-0.5,-0.55$ (each a s, $\left.-\mathrm{Si}\left(\mathrm{CH}_{3}\right)_{3}\right)$.

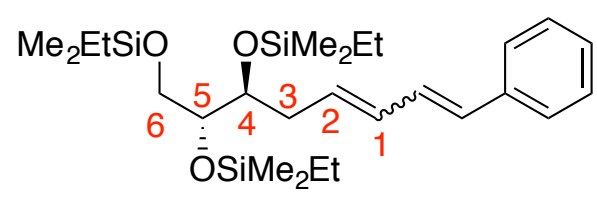

14

$\mathrm{Me}_{2}$ EtSi-styryl-2-deoxyribose diene (14): Prepared and used as a 76:24 E/Z mixture. ${ }^{1} \mathrm{H}$ NMR (500 MHz, $\left.\mathrm{CDCl}_{3}\right): \delta 7.44-7.37(\mathrm{~m}, 4 \mathrm{H}, \mathrm{Ph}, E, E / E, Z), 7.35-7.27(\mathrm{~m}, 4 \mathrm{H}, \mathrm{Ph}$, $E, E / E, Z$ ), 7.25-7.17 (m, Ph, 2H, E,E/E,Z), 7.07 (ddd, $J=15.6,11.0,1.2 \mathrm{~Hz}, 1 \mathrm{H}, E, Z$ ), 6.77 (dd, $J=15.6,10.5 \mathrm{~Hz}, 1 \mathrm{H}, E / E), 6.53(\mathrm{~d}, J=15.6 \mathrm{~Hz}, 1 \mathrm{H}, E, Z), 6.45$ (d, $J=15.6$ $\mathrm{Hz}, 1 \mathrm{H}, E / E), 6.24$ (dd, $J=15.6,10.3 \mathrm{~Hz}, 2 \mathrm{H}, E, E / E, Z), 5.83$ (dt, $J=15.0,7.4 \mathrm{~Hz}, 1 \mathrm{H}$, $E / E), 5.60(\mathrm{dt}, J=10.7,7.7 \mathrm{~Hz}, 1 \mathrm{H}, E, Z), 3.80-3.76(\mathrm{~m}, 1 \mathrm{H}, E, Z), 3.76-3.72(\mathrm{~m}, 1 \mathrm{H}$, $E / E), 3.71-3.63(\mathrm{~m}, 3 \mathrm{H}, E, E / E, Z), 3.64-3.57$ (m, $1 \mathrm{H}, E, E), 3.54-3.46(\mathrm{~m}, 2 \mathrm{H}, E, E / E, Z)$, 2.58-2.44 (m, 2H, E,Z), 2.44-2.35 (m, 1H, E,E), 2.36-2.25 (m, 1H, E,E), 1.00-0.88 (m, $\left.18 \mathrm{H},-\mathrm{Si}\left(\mathrm{CH}_{3}\right)_{2}\left(\mathrm{CH}_{2} \mathrm{CH}_{3}\right)\right), 0.68-0.52\left(\mathrm{~m}, 12 \mathrm{H},-\mathrm{Si}\left(\mathrm{CH}_{3}\right)_{2}\left(\mathrm{CH}_{2} \mathrm{CH}_{3}\right)\right), 0.12$ (s, 3H, $\left.\mathrm{Si}\left(\mathrm{CH}_{3}\right)_{2}\left(\mathrm{CH}_{2} \mathrm{CH}_{3}\right)\right), \quad 0.11 \quad\left(\mathrm{~s}, \quad 6 \mathrm{H}, \quad-\mathrm{Si}\left(\mathrm{CH}_{3}\right)_{2}\left(\mathrm{CH}_{2} \mathrm{CH}_{3}\right)\right), \quad 0.109 \quad$ (s, $\quad 6 \mathrm{H}, \quad-$ $\left.\mathrm{Si}\left(\mathrm{CH}_{3}\right)_{2}\left(\mathrm{CH}_{2} \mathrm{CH}_{3}\right)\right), \quad 0.09 \quad$ (s, $\left.\quad 9 \mathrm{H}, \quad-\mathrm{Si}\left(\mathrm{CH}_{3}\right)_{2} \quad\left(\mathrm{CH}_{2} \mathrm{CH}_{3}\right)\right), \quad 0.08 \quad(\mathrm{~s}, \quad 12 \mathrm{H}, \quad-$ $\left.\mathrm{Si}\left(\mathrm{CH}_{3}\right)_{2}\left(\mathrm{CH}_{2} \mathrm{CH}_{3}\right)\right) .{ }^{13} \mathrm{C}\left\{{ }^{1} \mathrm{H}\right\}$ NMR (151 MHz, $\left.\mathrm{CDCl}_{3}\right): \delta 137.64$ (s), 137.60 (s), 132.9 
(s), $132.2(\mathrm{~s}), 130.4(\mathrm{~s}), 130.3(\mathrm{~s}), 129.5(\mathrm{~s}), 129.3(\mathrm{~s}), 128.6(\mathrm{~s}), 127.3(\mathrm{~s}), 127.1(\mathrm{~s})$, $126.3(\mathrm{~s}), 126.2(\mathrm{~s}), 124.8(\mathrm{~s}), 76.7(\mathrm{~s}), 73.7(\mathrm{~s}), 73.6(\mathrm{~s}), 64.3(\mathrm{~s}), 64.25(\mathrm{~s}), 36.8(\mathrm{~s}), 31.8$ (s), 8.8, 8.7, 7.9, 7.0, 6.9, 6.8, -1.68,-1.7, -1.76, - 1.77, -1.8, -2.7 (each a s, $\mathrm{Si}\left(\mathrm{CH}_{3}\right)_{2}\left(\mathrm{CH}_{2} \mathrm{CH}_{3}\right),-\mathrm{Si}\left(\mathrm{CH}_{3}\right)_{2}\left(\mathrm{CH}_{2} \mathrm{CH}_{3}\right)$ or $\left.-\mathrm{Si}\left(\mathrm{CH}_{3}\right)_{2}\left(\mathrm{CH}_{2} \mathrm{CH}_{3}\right)\right)$. ( Note: Two sp ${ }^{2}$ carbon peaks, $1 \mathrm{C}-\mathrm{O}$ bond and in the silane region there are signals overlapping).

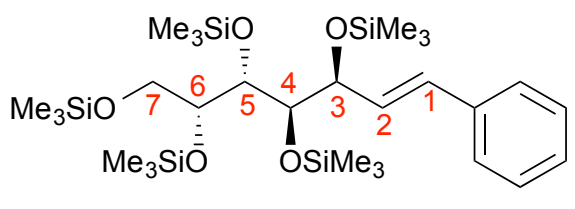

16

Me $_{3}$ Si-styrylgalactose (16): ${ }^{1} \mathrm{H}$ NMR $\left(600 \mathrm{MHz}, \mathrm{CD}_{2} \mathrm{Cl}_{2}\right): \delta 7.44-7.40(\mathrm{~m}, 2 \mathrm{H}), 7.39-$ $7.34(\mathrm{~m}, 2 \mathrm{H}), 7.31-7.27(\mathrm{~m}, 1 \mathrm{H}), 6.63\left(\mathrm{~d}, J_{\mathrm{H} 1, \mathrm{H} 2}=16.0 \mathrm{~Hz}, 1 \mathrm{H}, \mathrm{H}_{1}\right), 6.37\left(\mathrm{dd}, J_{\mathrm{H} 2, \mathrm{H} 1}=\right.$ $\left.16.0 \mathrm{~Hz}, J_{\mathrm{H} 2, \mathrm{H} 3}=6.7 \mathrm{~Hz}, 1 \mathrm{H}, \mathrm{H}_{2}\right), 4.56\left(\mathrm{ddd}, J_{\mathrm{H} 3, \mathrm{H} 2} \& J_{\mathrm{H} 3, \mathrm{H} 4}=6.9 \mathrm{~Hz}, J_{\mathrm{H} 3, \mathrm{H} 1}=1.3 \mathrm{~Hz}\right.$, $\left.1 \mathrm{H}, \mathrm{H}_{3}\right), 3.85\left(\mathrm{dd}, J_{\mathrm{H} 5, \mathrm{H} 6}=5.3 \mathrm{~Hz}, J_{\mathrm{H} 5, \mathrm{H} 4}=2.9 \mathrm{~Hz}, 1 \mathrm{H}, \mathrm{H}_{5}\right), 3.82\left(\mathrm{ddd}, J_{\mathrm{H} 6, \mathrm{H} 5}=5.5, J_{\mathrm{H} 6, \mathrm{H} 7}\right.$ $\left.\& J_{\mathrm{H} 6, \mathrm{H} 7}=3.4 \mathrm{~Hz}, 1 \mathrm{H}, \mathrm{H}_{6}\right), 3.79-3.76\left(\mathrm{~m}, 2 \mathrm{H}, \mathrm{H}_{7}\right), 3.69\left(\mathrm{dd}, J_{\mathrm{H} 4, \mathrm{H} 3}=7.0 \mathrm{~Hz}, J_{\mathrm{H} 4, \mathrm{H} 5}=2.9\right.$ $\left.\mathrm{Hz}, 1 \mathrm{H}, \mathrm{H}_{4}\right), 0.20-0.13\left(\mathrm{~m}, 45 \mathrm{H},-\mathrm{Si}\left(\mathrm{CH}_{3}\right)_{3}\right) .{ }^{13} \mathrm{C}\left\{{ }^{1} \mathrm{H}\right\} \operatorname{NMR}\left(151 \mathrm{MHz}, \mathrm{CD}_{2} \mathrm{Cl}_{2}\right): \delta 137.3$ (s, Ph), $131.1\left(\mathrm{~s}, \mathrm{C}_{2}\right), 130.8\left(\mathrm{~s}, \mathrm{C}_{1}\right), 128.5$ (s, Ph), $127.3(\mathrm{~s}, \mathrm{Ph}), 126.3$ (s, Ph), $80.0\left(\mathrm{~s}, \mathrm{C}_{4}\right)$, $76.1\left(\mathrm{~s}, \mathrm{C}_{6}\right), 74.4\left(\mathrm{~s}, \mathrm{C}_{3}\right), 73.5\left(\mathrm{~s}, \mathrm{C}_{5}\right), 64.4\left(\mathrm{~s}, \mathrm{C}_{7}\right), 0.6,0.34,0.31,0.2,-0.8$ (each a s, $\left.\mathrm{Si}\left(\mathrm{CH}_{3}\right)_{3}\right)$.

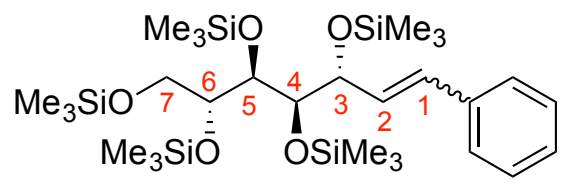

17

Me $_{3}$ Si-styrylmannose (17): Prepared and used as a $33: 67 \mathrm{E} / \mathrm{Z}$ mixture. ${ }^{1} \mathrm{H}$ NMR (600 $\left.\mathrm{MHz}, \mathrm{CDCl}_{3}\right): \delta$ 7.43-7.40 (m, 2H, Ph, Z), 7.39-7.37 (m, 2H, Ph, E), 7.34-7.31 (m, 4H, $E / Z), 7.25-7.23(\mathrm{~m}, 2 \mathrm{H}, E / Z) 6.63\left(\mathrm{~d}, J_{\mathrm{H} 1, \mathrm{H} 2}=11.7 \mathrm{~Hz}, 1 \mathrm{H}, \mathrm{H}_{1}, Z\right), 6.50\left(\mathrm{~d}, J_{\mathrm{H} 1, \mathrm{H} 2}=16.0\right.$ $\left.\mathrm{Hz}, 1 \mathrm{H}, \mathrm{H}_{1}, E\right), 6.27\left(\mathrm{dd}, J_{\mathrm{H} 2, \mathrm{H} 1}=16.0 \mathrm{~Hz}, J_{\mathrm{H} 2, \mathrm{H} 3}=7.4 \mathrm{~Hz}, 1 \mathrm{H}, \mathrm{H}_{2}, E\right), 5.68\left(\mathrm{dd}, J_{\mathrm{H} 2, \mathrm{H} 1}=\right.$ $\left.11.7 \mathrm{~Hz}, J_{\mathrm{H} 2, \mathrm{H} 3}=9.8 \mathrm{~Hz}, 1 \mathrm{H}, \mathrm{H}_{2}, Z\right), 4.74\left(\mathrm{dd}, J_{\mathrm{H} 3, \mathrm{H} 2}=9.8 \mathrm{~Hz}, J_{\mathrm{H} 3, \mathrm{H} 4}=7.6 \mathrm{~Hz}, 1 \mathrm{H}, \mathrm{H}_{3}, Z\right)$, $4.36\left(\mathrm{dd}, J_{\mathrm{H} 3, \mathrm{H} 2}=7.4 \mathrm{~Hz}, J_{\mathrm{H} 3, \mathrm{H} 4}=3.7 \mathrm{~Hz}, 1 \mathrm{H}, \mathrm{H}_{3}, E\right), 3.93-3.68\left(\mathrm{~m}, 8 \mathrm{H}, \mathrm{H}_{5}, \mathrm{H}_{6}, \mathrm{H}_{7}, E / Z\right)$, 3.55-3.45 (m, 2H, H4, E/Z), 0.13 (s, 9H, -Si( $\left.\left.\mathrm{CH}_{3}\right)_{3}\right), 0.12$ (s, 9H, $\left.-\mathrm{Si}\left(\mathrm{CH}_{3}\right)_{3}\right), 0.116$ (s, 9H, $\left.-\mathrm{Si}\left(\mathrm{CH}_{3}\right)_{3}\right), 0.112\left(\mathrm{~s}, 18 \mathrm{H},-\mathrm{Si}\left(\mathrm{CH}_{3}\right)_{3}\right), 0.107\left(\mathrm{~s}, 18 \mathrm{H},-\mathrm{Si}\left(\mathrm{CH}_{3}\right)_{3}\right), 0.105$ (s, 9H, $\left.-\mathrm{Si}\left(\mathrm{CH}_{3}\right)_{3}\right)$, $0.09\left(\mathrm{~s}, 18 \mathrm{H},-\mathrm{Si}\left(\mathrm{CH}_{3}\right)_{3}\right), 0.08\left(\mathrm{~s}, 9 \mathrm{H},-\mathrm{Si}\left(\mathrm{CH}_{3}\right)_{3}\right) \cdot{ }^{13} \mathrm{C}\left\{{ }^{1} \mathrm{H}\right\}$ NMR $\left(151 \mathrm{MHz}, \mathrm{CDCl}_{3}\right): \delta$ $137.0(\mathrm{~s}), 136.6(\mathrm{~s}), 133.5(\mathrm{~s}), 132.4(\mathrm{~s}), 131.7$ (s), $130.0(\mathrm{~s}), 129.1(\mathrm{~s}), 128.5(\mathrm{~s}), 128.1$ (s), $127.4(\mathrm{~s}), 127.1(\mathrm{~s}), 126.5(\mathrm{~s}), 77.7(\mathrm{~s}), 75.9(\mathrm{~s}), 75.29(\mathrm{~s}), 75.27(\mathrm{~s}), 73.9(\mathrm{~s}), 69.0(\mathrm{~s})$, $64.4(\mathrm{~s}), 63.3(\mathrm{~s}), 1.2,1.1,1.0,0.98,0.92,0.9,0.7,0.6,-0.4,-0.6$ (each a s, $\left.-\mathrm{Si}\left(\mathrm{CH}_{3}\right)_{3}\right)$. (*Note: two of the $Z / E$-olefin $\mathrm{C}-\mathrm{O}$ bonds are under solvent in the carbon NMR.) 


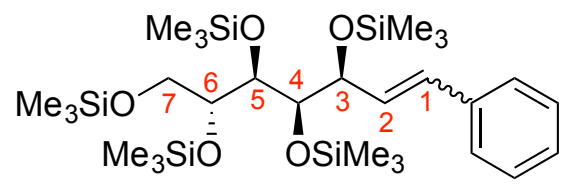

18

Me $_{3}$ Si-styrylglucose (18): Prepared and used as a 67:33 $E / Z$ mixture. ${ }^{1} \mathrm{H}$ NMR (600 $\mathrm{MHz}, \mathrm{CDCl}_{3}$ ): $\delta$ 7.41-7.36 (m, 4H, Ph, E/Z), 7.35-7.30 (m, 4H, Ph, E/Z), 7.28-7.21 (m, 2H, Ph, E/Z), 6.59 (d, $J=11.9 \mathrm{~Hz}, 1 \mathrm{H}, Z), 6.54$ (dd, $J=16.1,1.4 \mathrm{~Hz}, 1 \mathrm{H}, E), 6.38$ (dd, $J$ $=16.0,5.7 \mathrm{~Hz}, 1 \mathrm{H}, E), 5.68(\mathrm{dd}, J=11.9,9.8 \mathrm{~Hz}, 1 \mathrm{H}, Z), 4.67$ (ddd, $J=9.8,4.9,0.9 \mathrm{~Hz}$, $1 \mathrm{H}, Z), 4.41$ (app td, $J=5.5,1.5 \mathrm{~Hz}, 1 \mathrm{H}, E), 3.98$ (dt, $J=7.4,2.9 \mathrm{~Hz}, 1 \mathrm{H}, E), 3.94$ (ddd, $J$ $=7.2,4.3,2.9 \mathrm{~Hz}, 1 \mathrm{H}, Z), 3.83(\mathrm{dd}, J=5.5,4.3 \mathrm{~Hz}, 1 \mathrm{H}, Z), 3.77-3.71(\mathrm{~m}, 2 \mathrm{H}, E / Z), 3.66$ (dd, $J=6.2,5.2 \mathrm{~Hz}, 1 \mathrm{H}, E), 3.49-3.45(\mathrm{~m}, 1 \mathrm{H}, E / Z), 0.16\left(\mathrm{~s}, 9 \mathrm{H},-\mathrm{Si}\left(\mathrm{CH}_{3}\right)_{3}\right), 0.15(\mathrm{~s}, 9 \mathrm{H}$, $\left.-\mathrm{Si}\left(\mathrm{CH}_{3}\right)_{3}\right), 0.147$ (s, 9H, $\left.-\mathrm{Si}\left(\mathrm{CH}_{3}\right)_{3}\right), 0.14\left(\mathrm{~s}, 9 \mathrm{H},-\mathrm{Si}\left(\mathrm{CH}_{3}\right)_{3}\right), 0.13\left(\mathrm{~s}, 9 \mathrm{H},-\mathrm{Si}\left(\mathrm{CH}_{3}\right)_{3}\right), 0.11$ (s, 9H, -Si $\left.\left(\mathrm{CH}_{3}\right)_{3}\right), 0.10$ (s, 9H, -Si $\left.\left(\mathrm{CH}_{3}\right)_{3}\right), 0.09$ (s, 9H, -Si $\left.\left(\mathrm{CH}_{3}\right)_{3}\right), 0.07$ (s, 9H, $\left.\mathrm{Si}\left(\mathrm{CH}_{3}\right)_{3}\right), 0.02\left(\mathrm{~s}, 9 \mathrm{H},-\mathrm{Si}\left(\mathrm{CH}_{3}\right)_{3}\right) .{ }^{13} \mathrm{C}\left\{{ }^{1} \mathrm{H}\right\} \mathrm{NMR}\left(151 \mathrm{MHz}, \mathrm{CDCl}_{3}\right): \delta 137.1$ (s), 136.9 (s), 132.5 (s), 131.4 (s), 130.2 (s), 129.7 (s), 128.9 (s), 128.5 (s), 128.1 (s), 127.2 (s), 127.1 (s), 126.5 (s), 77.2 (s), 76.7 (s), 76.4 (s), 75.8 (s), 75.1 (s), 74.8 (s), 74.3 (s), 70.0 $(\mathrm{s}), 64.0(\mathrm{~s}), 63.8(\mathrm{~s}), 1.05,1.03,0.9,0.84,0.83,0.6,0.5,0.4,-0.5,-0.6$ (each a s, $\left.\mathrm{Si}\left(\mathrm{CH}_{3}\right)_{3}\right)$.

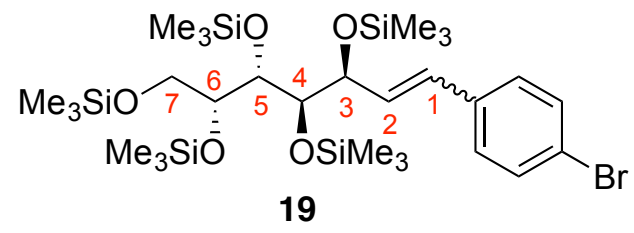

Me $_{3}$ Si-para-bromo-styrylgalactose (19): Prepared and used as a 63:27 E/Z mixture. ${ }^{1} \mathrm{H}$ NMR (600 MHz, $\left.\mathrm{CDCl}_{3}\right): \delta 7.44(\mathrm{~d}, J=8.4 \mathrm{~Hz}, 2 \mathrm{H}, \mathrm{Ph}, E), 7.43(\mathrm{~d}, J=8.4 \mathrm{~Hz}, 2 \mathrm{H}, \mathrm{Ph}$, $Z$ ), 7.36 (d, $J=8.4 \mathrm{~Hz}, 2 \mathrm{H}, \mathrm{Ph}, Z), 7.22$ (d, $J=8.4 \mathrm{~Hz}, 2 \mathrm{H}, \mathrm{Ph}, E), 6.51$ (d, $J=16.2 \mathrm{~Hz}$, $1 \mathrm{H}, E), 6.40(\mathrm{~d}, J=12.0 \mathrm{~Hz}, 1 \mathrm{H}, Z), 6.30(\mathrm{dd}, J=16.2,6.6 \mathrm{~Hz}, 1 \mathrm{H}, E), 5.79$ (dd, $J=$ 12.0, 10.2 Hz, 1H, Z), $4.74(\mathrm{dd}, J=9.6,4.8 \mathrm{~Hz}, 1 \mathrm{H}, Z), 4.49$ (t, $J=6.6 \mathrm{~Hz}, 1 \mathrm{H}, E), 3.89-$ $3.83(\mathrm{~m}, 2 \mathrm{H}, Z), 3.76-3.73(\mathrm{~m}, 5 \mathrm{H}, E / Z), 3.66(\mathrm{dd}, J=10.8,4.8 \mathrm{~Hz}, 1 \mathrm{H}, Z), 3.64-3.63$ $(\mathrm{m}, 1 \mathrm{H}, E), 3.61(\mathrm{dd}, J=10.8,4.2 \mathrm{~Hz}, 1 \mathrm{H}, Z), 0.16\left(\mathrm{~s}, 9 \mathrm{H},-\mathrm{Si}\left(\mathrm{CH}_{3}\right)_{3}\right), 0.13(\mathrm{~s}, 9 \mathrm{H}$, $\left.\mathrm{Si}\left(\mathrm{CH}_{3}\right)_{3}\right), 0.13\left(\mathrm{~s}, 9 \mathrm{H},-\mathrm{Si}\left(\mathrm{CH}_{3}\right)_{3}\right), 0.12\left(\mathrm{~s}, 18 \mathrm{H},-\mathrm{Si}\left(\mathrm{CH}_{3}\right)_{3}\right), 0.111\left(\mathrm{~s}, 18 \mathrm{H},-\mathrm{Si}\left(\mathrm{CH}_{3}\right)_{3}\right)$, 0.107 (s, 9H, -Si $\left.\left(\mathrm{CH}_{3}\right)_{3}\right), 0.08\left(\mathrm{~s}, 9 \mathrm{H},-\mathrm{Si}\left(\mathrm{CH}_{3}\right)_{3}\right),-0.7\left(\mathrm{~s}, 9 \mathrm{H},-\mathrm{Si}\left(\mathrm{CH}_{3}\right)_{3}\right) .{ }^{13} \mathrm{C}\left\{{ }^{1} \mathrm{H}\right\} \mathrm{NMR}$ (151 MHz, $\mathrm{CDCl}_{3}$ ): $\delta 136.4$ (s), 135.9 (s), 134.0 (s), 132.0 (s), 131.8 (s), 131.2 (s), 131.0 (s), 129.8 (s), 128.7 (s), 128.0 (s), 121.2 (s), 80.2 (s), 76.7 (s), 76.5 (s), 74.7 (s), 74.1 (s), 73.5 (s), 69.4 (s), 64.9 (s), 64.7 (s), 1.12, 1.05, 1.02, 0.95, 0.81, 0.75, 0.7, 0.6, -0.19, -0.20 (each a $\left.\mathrm{s},-\mathrm{Si}\left(\mathrm{CH}_{3}\right)_{3}\right)$. (*Note: One $\mathrm{C}-\mathrm{O}$ carbon and one $\mathrm{sp}^{2}$ carbon are overlapping for $E / Z$ isomers). 


\section{Representative preparation of deoxygentated substrates and silane deprotection of carbohydrate derivatives}

Cyclopropane formation from styryl carbohydrate substrate:

In a nitrogen-filled glovebox, a 1-dram vial was charged with $\mathrm{B}\left(\mathrm{C}_{6} \mathrm{~F}_{5}\right)_{3}(5.9 \mathrm{mg}, 0.012$ mmol, 0.11 equiv.) then triphenylsilane (28.6 mg, $0.110 \mathrm{mmol}, 1.02$ equiv.), then $\mathrm{Me}_{3} \mathrm{Si}_{-}$ styryl 2-deoxygalactose $3(56.7 \mathrm{mg}, 0.108 \mathrm{mmol}, 1.00$ equiv.) and a magnetic stir bar. By syringe $\mathrm{CH}_{2} \mathrm{Cl}_{2}(0.5 \mathrm{~mL})$ was added. The vial was sealed with a septa cap and removed from the glovebox and allowed to stir at $22{ }^{\circ} \mathrm{C}$. After 16 hours, $\mathrm{NEt}_{3}(0.05 \mathrm{~mL})$ was added by syringe through the septa and the stir bar was removed, rinsing with $\mathrm{CH}_{2} \mathrm{Cl}_{2}(2$ $\mathrm{mL}$ ). The quenched reaction mixture was concentrated under vacuum and then loaded onto an alumina plug (neutral, $10 \% \mathrm{H}_{2} \mathrm{O} ; 2 \times 1 \mathrm{~cm}$ ) with $100 \%$ hexanes $(1.5 \mathrm{~mL})$ then 95:5 hexanes:diethyl ether $(1.5 \mathrm{~mL})$, eluting with $95: 5$ hexanes:diethyl ether $(10 \mathrm{~mL})$ into a tared vial. The solution was concentrated under vacuum to afford a colorless oil (66.7 $\mathrm{mg}$ ) that was subsequently deprotected vide infra.

\section{Cyclopentane formation from styryl carbohydrate substrate}

In a nitrogen-filled glovebox, a 1-dram vial was charged with $\mathrm{Me}_{3} \mathrm{Si}$-styryl galactose 16 (61.4 mg, $0.0998 \mathrm{mmol}, 1.00$ equiv.) and a magnetic stir bar. A separate 1-dram vial was charged with $\mathrm{B}\left(\mathrm{C}_{6} \mathrm{~F}_{5}\right)_{3}(5.0 \mathrm{mg}, 0.0098 \mathrm{mmol}, 0.098$ equiv. $)$ and $\mathrm{CH}_{2} \mathrm{Cl}_{2}(0.5 \mathrm{~mL})$. The vials were sealed with a septa cap and removed from the glovebox. The vials were allowed to cool to $-30{ }^{\circ} \mathrm{C}$ (dry ice/acetone bath). The $\mathrm{B}\left(\mathrm{C}_{6} \mathrm{~F}_{5}\right)_{3}$ solution was transferred to the substrate by syringe and allowed to stir as the dimethylethylsilane $(50.0 \mu \mathrm{L}, 0.379$ mmol, 3.79 equiv.) was added by microliter syringe under positive $\mathrm{N}_{2}$ pressure. The vial cap was wrapped with electrical tape and transferred to a $-30{ }^{\circ} \mathrm{C}$ cryobath and allowed to stir. After 12 hours, $\mathrm{NEt}_{3}(0.05 \mathrm{~mL})$ was added by syringe through the septum and the stir bar was removed, rinsing with $\mathrm{CH}_{2} \mathrm{Cl}_{2}(0.2 \mathrm{~mL})$. The quenched reaction mixture was concentrated under vacuum and then loaded onto an alumina plug (neutral, $10 \% \mathrm{H}_{2} \mathrm{O} ; 2 \mathrm{x}$ $1 \mathrm{~cm})$ with $100 \%$ hexanes $(1.5 \mathrm{~mL})$ then $95: 5$ hexanes:diethyl ether $(1.5 \mathrm{~mL})$, eluting with 95:5 hexanes:diethyl ether $(10 \mathrm{~mL})$ into a tared vial. The solution was concentrated under vacuum to afford a colorless oil $(71.2 \mathrm{mg})$ that was subsequently deprotected vide infra.

\section{Deprotection to alcohol:}

The vial containing the silyl substrate was equipped with a stir bar and charged with Dowex ${ }^{\circledR}$ resin $(\sim 10$ beads). Methanol $(2.0 \mathrm{~mL})$ was added by syringe and the resulting solution was allowed to stir at $25{ }^{\circ} \mathrm{C}$. After $2-3 \mathrm{~h}$, the solution was passed through sand $(0.1 \mathrm{~cm})$ to remove the Dowe ${ }^{\circledR}$ beads into a tared vial, rinsing with $100 \%$ methanol. Products were purified by silica gel chromatography $(4 \times 1 \mathrm{~cm}$ column) using gradient 
elution $\left(100 \% \mathrm{CH}_{2} \mathrm{Cl}_{2}\right.$ to $20: 1$ to $15: 1$ to $10: 1$ to $\left.9: 1 \mathrm{CH}_{2} \mathrm{Cl}_{2}: \mathrm{MeOH}\right)$ to afford a white solid.

\section{Preparation of carbocycle product 6:}
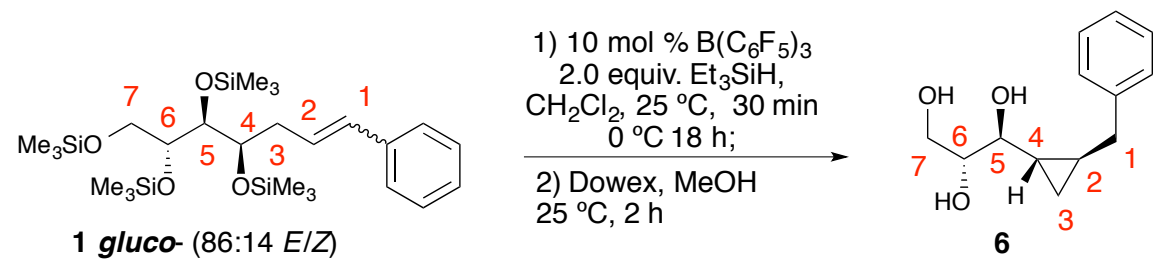

In a nitrogen-filled glovebox, a 1-dram vial was charged with $\mathrm{Me}_{3} \mathrm{Si}$-styryl 2deoxyglucose 1 (52.7 mg, $0.100 \mathrm{mmol}, 1.00$ equiv.) and a magnetic stir bar. A separate 1dram vial was charged with $\mathrm{B}\left(\mathrm{C}_{6} \mathrm{~F}_{5}\right)_{3}(5.1 \mathrm{mg}, 0.010 \mathrm{mmol}, 0.10$ equiv. $)$ and $\mathrm{CH}_{2} \mathrm{Cl}_{2}(0.5$ $\mathrm{mL})$. The contents of the vials were mixed in the glovebox at $25^{\circ} \mathrm{C}$ and sealed. After 30 minutes had passed the vials were placed into a cryobath set at $0{ }^{\circ} \mathrm{C}$ and allowed to stir. After 18 hours, $\mathrm{NEt}_{3}(0.05 \mathrm{~mL})$ was added by syringe through the septa and the stir bar was removed, rinsing with $\mathrm{CH}_{2} \mathrm{Cl}_{2}(2 \mathrm{~mL})$. The quenched reaction mixture was concentrated under vacuum and then loaded onto an alumina plug (neutral, $10 \% \mathrm{H}_{2} \mathrm{O} ; 2 \mathrm{x}$ $1 \mathrm{~cm})$ with $100 \%$ hexanes $(1.5 \mathrm{~mL})$ then $95: 5$ hexanes:diethyl ether $(1.5 \mathrm{~mL})$, eluting with 95:5 hexanes:diethyl ether $(10 \mathrm{~mL})$ into a tared vial. The solution was concentrated under vacuum to afford a colorless oil $(63.0 \mathrm{mg})$. The vial containing the silyl substrate was equipped with a stir bar and charged with Dowex ${ }^{\circledR}$ resin ( 10 beads). Methanol (2.0 $\mathrm{mL}$ ) was added by syringe and the resulting solution was allowed to stir at $25{ }^{\circ} \mathrm{C}$. After 2 $\mathrm{h}$, the solution was passed through sand $(0.1 \mathrm{~cm})$ to remove the Dowex ${ }^{\circledR}$ beads into a tared vial, rinsing with $100 \%$ methanol. The product was purified by silica gel chromatography ( $4 \times 1 \mathrm{~cm}$ column) using gradient elution $\left(100 \% \mathrm{CH}_{2} \mathrm{Cl}_{2}\right.$ to $50: 1$ to $45: 1$ to $\left.40: 1 \mathrm{CH}_{2} \mathrm{Cl}_{2}: \mathrm{MeOH}\right)$ to afford carbocycle from $\mathrm{Me}_{3} \mathrm{Si}$-styryl 2-deoxyglucose 6 as a white solid (18.2 $\mathrm{mg}, 82 \%$ overall yield over 2 steps, $0.0819 \mathrm{mmol}$, >98:2 dr). Note: Stereochemistry assigned by NOE between $\mathrm{H}_{4}$ and $\mathrm{H}_{1}$.

\section{Data for 6}

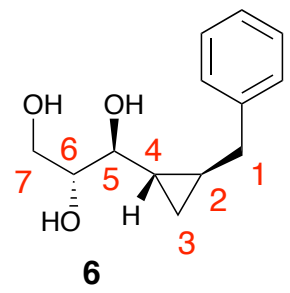

(1S,2R)-1-((1S,2R)-2-benzylcyclopropyl)propane-1,2,3-triol (6): ${ }^{1} \mathrm{H}$ NMR (600 MHz, $\left.\mathrm{CD}_{3} \mathrm{OD}\right): \delta 7.27-7.22(\mathrm{~m}, 4 \mathrm{H}, \mathrm{Ph}), 7.17-7.14(\mathrm{~m}, 1 \mathrm{H}, \mathrm{Ph}), 3.66\left(\mathrm{dd}, J_{\mathrm{H} 7, \mathrm{H} 7}=10.2 \mathrm{~Hz}\right.$, $\left.J_{\mathrm{H} 7, \mathrm{H} 6}=3.0 \mathrm{~Hz}, 1 \mathrm{H}, \mathrm{H}_{7}\right), 3.58-3.54\left(\mathrm{~m}, 2 \mathrm{H}, \mathrm{H}_{6} \& \mathrm{H}_{7}\right), 3.07\left(\mathrm{dd}, J_{\mathrm{H} 5, \mathrm{H} 4}=7.8 \mathrm{~Hz}, J_{\mathrm{H} 5, \mathrm{H} 6}=\right.$ 
$\left.4.2 \mathrm{~Hz}, 1 \mathrm{H}, \mathrm{H}_{5}\right), 2.73\left(\mathrm{dd}, J_{\mathrm{H} 1, \mathrm{H} 1}{ }^{\prime}=14.4 \mathrm{~Hz}, J_{\mathrm{H} 1 \mathrm{H} 2}=6.0 \mathrm{~Hz}, 1 \mathrm{H}, \mathrm{H}_{1}\right), 2.50\left(\mathrm{dd}, J_{\mathrm{H} 1}{ }^{\prime} \mathrm{H} 1=\right.$ $\left.14.4 \mathrm{~Hz}, J_{\mathrm{H} 1}, \mathrm{H}_{2}=7.2 \mathrm{~Hz}, 1 \mathrm{H}, \mathrm{H}_{1}{ }^{\prime}\right), 1.03-0.91\left(\mathrm{~m}, 1 \mathrm{H}, \mathrm{H}_{2}\right), 0.95-0.91\left(\mathrm{~m}, 1 \mathrm{H}, \mathrm{H}_{4}\right), 0.57$ (ddd, $\left.J_{\mathrm{H} 3, \mathrm{H} 3^{\prime}}=9.0 \mathrm{~Hz}, J_{\mathrm{H} 3, \mathrm{H} 2}=J_{\mathrm{H} 3, \mathrm{H} 4}=4.8 \mathrm{~Hz}, 1 \mathrm{H}, \mathrm{H}_{3}\right), 0.43\left(\mathrm{ddd}, J_{\mathrm{H} 3^{\prime}, \mathrm{H} 3}=9.0 \mathrm{~Hz}, J_{\mathrm{H} 3}, \mathrm{H} 2\right.$ $\left.=J_{\mathrm{H} 3^{\prime}, \mathrm{H} 4}=5.4 \mathrm{~Hz}, 1 \mathrm{H}, \mathrm{H}_{3}{ }^{\prime}\right) \cdot{ }^{13} \mathrm{C}\left\{{ }^{1} \mathrm{H}\right\}$ NMR $\left(151 \mathrm{MHz}, \mathrm{CD}_{3} \mathrm{OD}\right): \delta 142.8(\mathrm{~s}, \mathrm{Ph}), 129.5$ (s, Ph), 129.3 (s, Ph), 126.9 (s, Ph), 76.8 (s, C 5 ), 76.5 (s, $\mathrm{C}_{6}$ ), $64.4\left(\mathrm{~s}, \mathrm{C}_{7}\right), 40.3\left(\mathrm{~s}, \mathrm{C}_{1}\right)$, $21.6\left(\mathrm{~s}, \mathrm{C}_{4}\right), 18.9\left(\mathrm{~s}, \mathrm{C}_{2}\right), 9.8\left(\mathrm{~s}, \mathrm{C}_{3}\right)$. HRMS $\left(\mathrm{ESI}^{+}\right)$: Calcd for $\mathrm{C}_{13} \mathrm{H}_{18} \mathrm{O}_{3} \mathrm{Na}[\mathrm{M}+\mathrm{Na}]^{+}$: 245.1148; Found: 245.1153. Specific rotation: $[\alpha]^{\mathrm{D}} 24.9-35\left(\mathrm{c}=0.11 \mathrm{CH}_{3} \mathrm{OH}\right)$.

\section{Preparation of carbocycle product 7:}

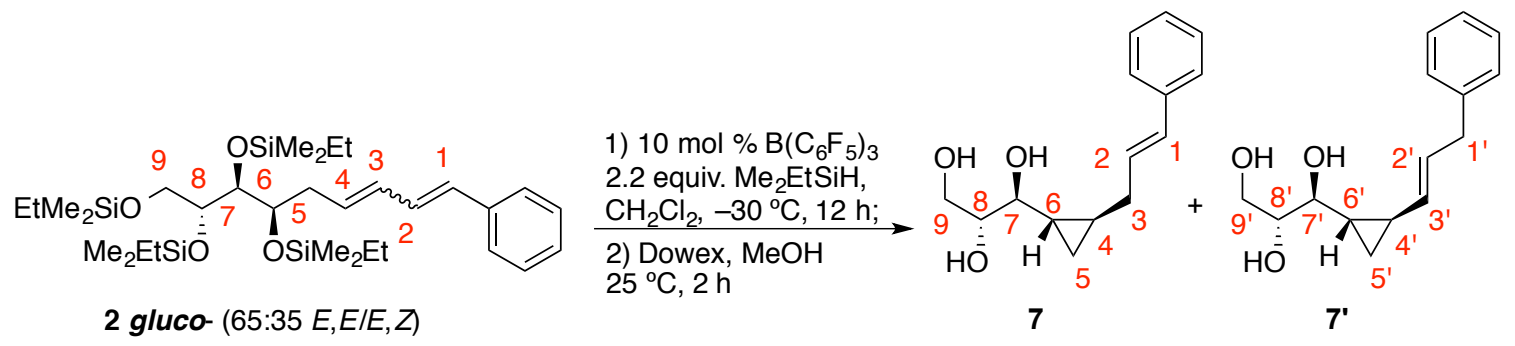

In a nitrogen-filled glovebox, a 1-dram vial was charged with $\mathrm{Me}_{2} \mathrm{EtSi}$-2-deoxyglucose diene $2(61.0 \mathrm{mg}, 0.100 \mathrm{mmol}, 1.00$ equiv.) and a magnetic stir bar. A separate 1-dram vial was charged with $\mathrm{B}\left(\mathrm{C}_{6} \mathrm{~F}_{5}\right)_{3}\left(5.1 \mathrm{mg}, 0.010 \mathrm{mmol}, 0.10\right.$ equiv.) and $\mathrm{CH}_{2} \mathrm{Cl}_{2}(0.5 \mathrm{~mL})$. The vials were sealed with a septa cap and removed from the glovebox. The vials were allowed to cool to $-30{ }^{\circ} \mathrm{C}$ (dry ice/acetone bath). The $\mathrm{B}\left(\mathrm{C}_{6} \mathrm{~F}_{5}\right)_{3}$ solution was transferred to the substrate by syringe and allowed to stir as dimethylethylsilane $(29.0 \mu \mathrm{L}, 0.220 \mathrm{mmol}$, 2.2 equiv.) was added by microliter syringe. The vial was wrapped with electrical tape and transferred to a $-30{ }^{\circ} \mathrm{C}$ cryobath and allowed to stir. After 12 hours, $\mathrm{NEt}_{3}(0.05 \mathrm{~mL})$ was added by syringe through the septa and the stir bar was removed, rinsing with $\mathrm{CH}_{2} \mathrm{Cl}_{2}(2 \mathrm{~mL})$. The quenched reaction mixture was concentrated under vacuum and then loaded onto an alumina plug (neutral, $10 \% \mathrm{H}_{2} \mathrm{O} ; 2 \times 1 \mathrm{~cm}$ ) with $100 \%$ hexanes $(1.5 \mathrm{~mL}$ ) then 95:5 hexanes:diethyl ether $(1.5 \mathrm{~mL})$, eluting with 95:5 hexanes:diethyl ether $(10 \mathrm{~mL})$ into a vial. The solution was concentrated under vacuum to afford a colorless oil. The vial containing the silyl substrate was equipped with a stir bar and charged with Dowex ${ }^{\circledR}$ resin ( $\sim 10$ beads). Methanol $(2.0 \mathrm{~mL})$ was added by syringe and the resulting solution was allowed to stir at $25{ }^{\circ} \mathrm{C}$. After $2 \mathrm{~h}$, the solution was passed through sand $(0.1 \mathrm{~cm})$ to remove the Dowex ${ }^{\circledR}$ beads into a tared vial, rinsing with $100 \%$ methanol. The product was purified by silica gel chromatography ( $4 \mathrm{x} 1 \mathrm{~cm}$ column) using gradient elution (100\% $\mathrm{CH}_{2} \mathrm{Cl}_{2}$ to $50: 1$ to $45: 1$ to $\left.40: 1 \mathrm{CH}_{2} \mathrm{Cl}_{2}: \mathrm{MeOH}\right)$ to afford carbocycle from $\mathrm{Me}_{2}$ EtSi-styryl-2-deoxyglucose 7 as a white solid (18.2 $\mathrm{mg}, 82 \%$ overall yield over 2 steps, $0.0819 \mathrm{mmol},>98: 2 \mathrm{dr}$, and $75: 25 \mathrm{C}_{3} / \mathrm{C}_{1}$ hydride addition). Note: Stereochemistry assigned by NOE between $\mathrm{H}_{3}$ and $\mathrm{H}_{6}$.

\section{Data for 7}




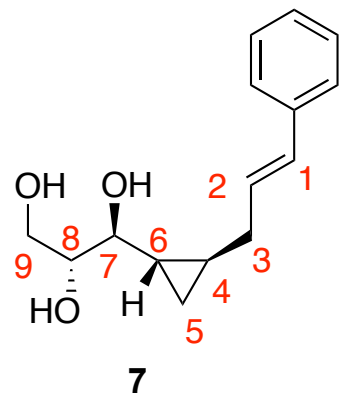

(1S,2R)-1-((1S,2S)-2-cinnamylcyclopropyl)propane-1,2,3-triol (7): ${ }^{1} \mathrm{H}$ NMR $\quad(600$ $\left.\mathrm{MHz}, \mathrm{CD}_{3} \mathrm{OD}\right): \delta$ 7.37-7.33 (m, 2H, Ph), 7.28-7.22 (m, 2H, Ph), 7.19-7.11 (m, 1H, Ph), $6.43\left(\mathrm{~d}, J_{\mathrm{H} 1, \mathrm{H} 2}=15.7 \mathrm{~Hz}, 1 \mathrm{H}, \mathrm{H}_{1}\right), 6.32\left(\mathrm{dt}, J_{\mathrm{H} 2, \mathrm{H} 1}=15.8 \mathrm{~Hz}, J_{\mathrm{H} 2, \mathrm{H} 3}=6.7 \mathrm{~Hz}, 1 \mathrm{H}, \mathrm{H}_{2}\right)$, $3.78-3.73\left(\mathrm{~m}, 1 \mathrm{H}, \mathrm{H}_{9}\right), 3.72-3.57\left(\mathrm{~m}, 2 \mathrm{H}, \mathrm{H}_{8} \& \mathrm{H}_{9}\right), 3.06\left(\mathrm{dd}, J_{\mathrm{H} 7, \mathrm{H} 8}=8.0 \mathrm{~Hz}, J_{\mathrm{H} 7, \mathrm{H} 6}=\right.$ $\left.4.5 \mathrm{~Hz}, 1 \mathrm{H}, \mathrm{H}_{7}\right), 2.26\left(\mathrm{dt}, J_{\mathrm{H} 3, \mathrm{H} 3},=13.9 \mathrm{~Hz}, J_{\mathrm{H} 3, \mathrm{H} 2}=J_{\mathrm{H} 3, \mathrm{H} 4}=6.4 \mathrm{~Hz}, 1 \mathrm{H}, \mathrm{H}_{3}\right), 2.11(\mathrm{dt}$, $\left.J_{\mathrm{H}^{3}, \mathrm{H} 3}=14.2 \mathrm{~Hz}, J_{\mathrm{H} 3^{\prime}, \mathrm{H} 2}=J_{\mathrm{H}^{3}, \mathrm{H} 4}=6.6, J_{\mathrm{H}^{3}, \mathrm{H} 4}=1.4 \mathrm{~Hz}, 1 \mathrm{H}, \mathrm{H}_{3}{ }^{\prime}\right), 0.95-0.82\left(\mathrm{~m}, 2 \mathrm{H}, \mathrm{H}_{4}\right.$ $\left.\& \mathrm{H}_{6}\right), 0.57\left(\mathrm{dt}, J_{\mathrm{H} 5, \mathrm{H} 5}=8.3 \mathrm{~Hz}, J_{\mathrm{H} 5, \mathrm{H} 4} \& J_{\mathrm{H} 5, \mathrm{H} 6}=4.7 \mathrm{~Hz}, 1 \mathrm{H}, \mathrm{H}_{5}\right), 0.39\left(\mathrm{dt}, J_{\mathrm{H} 5}{ }^{\mathrm{H} 5}=8.3\right.$ $\left.\mathrm{Hz}, J_{\mathrm{H} 5^{\prime}, \mathrm{H} 4} \& J_{\mathrm{H} 5^{\prime}, \mathrm{H} 6}=4.8 \mathrm{~Hz}, 1 \mathrm{H}, \mathrm{H}_{5}\right) .{ }^{13} \mathrm{C}\left\{{ }^{1} \mathrm{H}\right\} \mathrm{NMR}\left(151 \mathrm{MHz}, \mathrm{CD}_{3} \mathrm{OD}\right): \delta 137.8(\mathrm{~s}$, $\mathrm{Ph}), 130.1\left(\mathrm{~s}, \mathrm{C}_{1}\right), 129.0\left(\mathrm{~s}, \mathrm{C}_{2}\right), 128.1(\mathrm{~s}, \mathrm{Ph}), 126.5(\mathrm{~s}, \mathrm{Ph}), 125.6(\mathrm{~s}, \mathrm{Ph}), 75.5\left(\mathrm{~s}, \mathrm{C}_{8}\right)$, $75.3\left(\mathrm{~s}, \mathrm{C}_{7}\right), 63.2\left(\mathrm{~s}, \mathrm{C}_{9}\right), 36.4\left(\mathrm{~s}, \mathrm{C}_{3}\right), 20.2\left(\mathrm{~s}, \mathrm{C}_{6}\right), 16.0\left(\mathrm{~s}, \mathrm{C}_{4}\right), 8.1\left(\mathrm{~s}, \mathrm{C}_{5}\right)$. HRMS $\left(\mathrm{ESI}^{+}\right)$: Calcd for $\mathrm{C}_{15} \mathrm{H}_{20} \mathrm{O}_{3} \mathrm{Na}[\mathrm{M}+\mathrm{Na}]^{+}: 249.1485$; Found: 249.1488 . Specific rotation: $[\alpha]^{\mathrm{D}}{ }_{25.8}$ $-22.0\left(\mathrm{c}=1.47 \mathrm{CH}_{3} \mathrm{OH}\right)$.

\section{Data for 7}

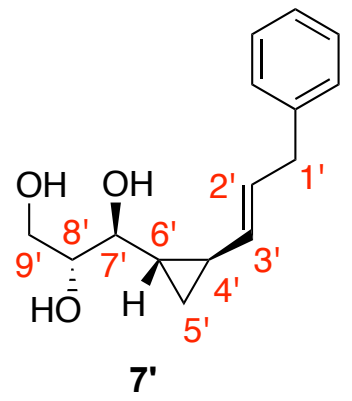

(1S,2R)-1-((1S,2S)-2-cinnamylcyclopropyl)propane-1,2,3-triol (7'): ${ }^{1} \mathrm{H}$ NMR $(600$ $\left.\mathrm{MHz}, \mathrm{CD}_{3} \mathrm{OD}\right): \delta$ 7.37-7.33 (m, 2H, Ph), 7.28-7.22 (m, 2H, Ph), 7.19-7.11 (m, 1H, Ph), $5.63\left(\mathrm{dt}, J_{\mathrm{H}^{2}{ }^{\prime} 3^{\prime}}=15.1 \mathrm{~Hz}, J_{\mathrm{H}^{\prime}{ }^{\prime} 1^{\prime}}=6.9 \mathrm{~Hz}, 1 \mathrm{H}, \mathrm{H}_{2}{ }^{\prime}\right), 5.12\left(\mathrm{ddt}, J_{\mathrm{H}^{3}, \mathrm{H}^{\prime}}=15.1 \mathrm{~Hz}, J_{\mathrm{H} 3^{\prime} \mathrm{H} 4^{\prime}}\right.$ $\left.=8.5 \mathrm{~Hz}, J_{\mathrm{H}^{\prime}{ }^{\prime} \mathrm{H}^{\prime}}=1.5 \mathrm{~Hz}, 1 \mathrm{H}, \mathrm{H}_{3^{\prime}}\right), 3.70\left(\mathrm{~m}, 1 \mathrm{H}, \mathrm{H}_{8^{\prime}}\right), 3.65-3.59\left(\mathrm{~m}, 2 \mathrm{H}, \mathrm{H}_{9}\right), 3.29(\mathrm{~d}$, $\left.J_{\mathrm{H}^{\prime}, \mathrm{H}{ }^{\prime}}=7.0 \mathrm{~Hz}, 2 \mathrm{H}, \mathrm{H}_{1}{ }^{\prime}\right), 3.08\left(\mathrm{dd}, J=8.2,4.5 \mathrm{~Hz}, 2 \mathrm{H}, \mathrm{H}_{7^{\prime}}\right), 1.38\left(\mathrm{tt}, J_{\mathrm{H}^{\prime}, \mathrm{H}^{\prime}}{ }^{\prime}=J_{\mathrm{H}^{\prime}{ }^{\prime} \mathrm{H} 5^{\prime}}=\right.$ $\left.8.5 \mathrm{~Hz}, J_{\mathrm{H}^{\prime}{ }^{\prime} 6^{\prime}}=J_{\mathrm{H} 4^{\prime} \mathrm{H} 5^{\prime}}=4.7 \mathrm{~Hz}, 1 \mathrm{H}, \mathrm{H}_{4}{ }^{\prime}\right), 1.06\left(\mathrm{tdd}, J_{\mathrm{H} 6^{\prime}, \mathrm{H} 7^{\prime}} \& J_{\mathrm{H} 6^{\prime}, \mathrm{H} 5^{\prime}}=8.3 \mathrm{~Hz}, J_{\mathrm{H} 6^{\prime}, \mathrm{H} 5^{\prime},}\right.$ $\left.=5.4 \mathrm{~Hz}, J_{\mathrm{H} 6^{\prime}, \mathrm{H} 4^{\prime}}=4.5 \mathrm{~Hz}, 1 \mathrm{H}, \mathrm{H}_{6^{\prime}}\right), 0.73\left(\mathrm{dt}, J_{\mathrm{H} 5^{\prime}, \mathrm{H} 5^{\prime}},=8.5 \mathrm{~Hz}, J_{\mathrm{H} 5^{\prime}, \mathrm{H} 4}, \& J_{\mathrm{H} 5^{\prime}, \mathrm{H} 6^{\prime}}=5.5\right.$ $\mathrm{Hz}, 1 \mathrm{H}, \mathrm{H}_{5}$ ) , 0.57-0.53 (m, 1H, $\left.\mathrm{H}_{5}{ }^{\prime}\right) .{ }^{13} \mathrm{C}\left\{{ }^{1} \mathrm{H}\right\} \mathrm{NMR}\left(151 \mathrm{MHz}, \mathrm{CD}_{3} \mathrm{OD}\right): \delta 142.2$ (s, $\mathrm{Ph}), 131.4\left(\mathrm{~s}, \mathrm{C}_{3}{ }^{\prime}\right), 129.3(\mathrm{~s}, \mathrm{Ph}), 128.5\left(\mathrm{~s}, \mathrm{C}_{2}{ }^{\prime}\right), 126.9(\mathrm{~s}, \mathrm{Ph}), 76.5\left(\mathrm{~s}, \mathrm{C}_{8}{ }^{\prime}\right), 76.3\left(\mathrm{~s}, \mathrm{C}_{7}\right)$, 
$64.5\left(\mathrm{~s}, \mathrm{C}_{9}{ }^{\prime}\right), 39.8\left(\mathrm{~s}, \mathrm{C}_{1}{ }^{\prime}\right), 22.9\left(\mathrm{~s}, \mathrm{C}_{6^{\prime}}\right), 20.2\left(\mathrm{~s}, \mathrm{C}_{4}\right), 11.1\left(\mathrm{~s}, \mathrm{C}_{5}{ }^{\prime}\right)$. (*Note: One aromatic carbon is overlapping with $\mathrm{S}_{\mathrm{N}} 2$ ' product).

\section{Preparation of carbocycle product 8:}

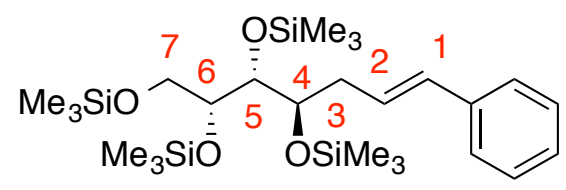

3 galacto- $(77: 23 \quad E / Z)$
1) $11 \mathrm{~mol} \% \mathrm{~B}\left(\mathrm{C}_{6} \mathrm{~F}_{5}\right)_{3}$

1.0 equiv. $\mathrm{Ph}_{3} \mathrm{SiH}$,

$\mathrm{CH}_{2} \mathrm{Cl}_{2}, 25^{\circ} \mathrm{C}, 16 \mathrm{~h}$;

2) Dowex, $\mathrm{MeOH}$

$25^{\circ} \mathrm{C}, 2 \mathrm{~h}$

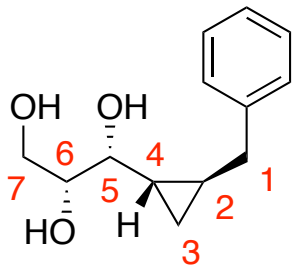

8

In a nitrogen-filled glovebox, a 1-dram vial was charged with $\mathrm{B}\left(\mathrm{C}_{6} \mathrm{~F}_{5}\right)_{3}(5.9 \mathrm{mg}, 0.012$ mmol, 0.11 equiv.) then triphenylsilane $\left(28.6 \mathrm{mg}, 0.110 \mathrm{mmol}, 1.02\right.$ equiv.), then $\mathrm{Me}_{3} \mathrm{Si}-$ styryl 2-deoxygalactose 3 (56.7 mg, $0.108 \mathrm{mmol}, 1.00$ equiv.) and a magnetic stir bar. By syringe $\mathrm{CH}_{2} \mathrm{Cl}_{2}(0.5 \mathrm{~mL})$ was added. The vial was sealed with a septa cap and removed from the glovebox and allowed to stir at $22{ }^{\circ} \mathrm{C}$. After 16 hours, $\mathrm{NEt}_{3}(0.05 \mathrm{~mL})$ was added by syringe through the septa and the stir bar was removed, rinsing with $\mathrm{CH}_{2} \mathrm{Cl}_{2}(2$ $\mathrm{mL})$. The quenched reaction mixture was concentrated under vacuum and then loaded onto an alumina plug (neutral, $10 \% \mathrm{H}_{2} \mathrm{O} ; 2 \times 1 \mathrm{~cm}$ ) with $100 \%$ hexanes $(1.5 \mathrm{~mL})$ then 95:5 hexanes:diethyl ether $(1.5 \mathrm{~mL})$, eluting with 95:5 hexanes:diethyl ether (10 $\mathrm{mL})$ into a tared vial. The solution was concentrated under vacuum to afford a colorless oil (66.7 $\mathrm{mg}$ ). The vial containing the silyl substrate was equipped with a stir bar and charged with Dowex $^{\circledR}$ resin ( $\sim 10$ beads). Methanol $(2.0 \mathrm{~mL})$ was added by syringe and the resulting solution was allowed to stir at $25^{\circ} \mathrm{C}$. After $2 \mathrm{~h}$, the solution was passed through sand $(0.1$ $\mathrm{cm})$ to remove the Dowex ${ }^{\circledR}$ beads into a tared vial, rinsing with $100 \%$ methanol. The product was purified by silica gel chromatography (4 x $1 \mathrm{~cm}$ column) using gradient elution ( $100 \% \mathrm{CH}_{2} \mathrm{Cl}_{2}$ to $50: 1$ to $49: 1$ to $48: 1$ to $47: 1$ to $46: 1$ to $\left.45: 1 \mathrm{CH}_{2} \mathrm{Cl}_{2}: \mathrm{MeOH}\right)$ to afford cyclopropane from $\mathrm{Me}_{3} \mathrm{Si}$-styryl 2-deoxygalactose 8 as a white solid (22.9 $\mathrm{mg}$, $95 \%$ overall yield over 2 steps, $0.103 \mathrm{mmol},>98: 2 \mathrm{dr}$ ). Note: Stereochemistry assigned by $\mathrm{NOE}$ between $\mathrm{H}_{4}$ and $\mathrm{H}_{1}$.

\section{Data for 8}

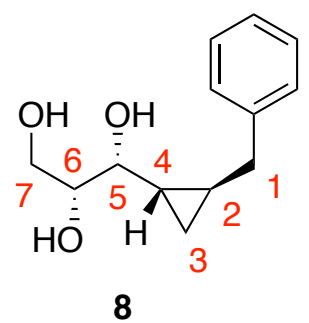


(1R,2R)-1-((1S,2R)-2-benzylcyclopropyl)propane-1,2,3-triol (8): ${ }^{1} \mathrm{H}$ NMR (600 MHz, $\left.\mathrm{CD}_{3} \mathrm{OD}\right): \delta$ 7.31-7.25 (m, 4H, Ar), 7.18-7.15 (m, 1H, Ar), 3.70-3.67 (m, 1H, $\left.\mathrm{H}_{7}\right), 3.59-$ $3.55\left(\mathrm{~m}, 2 \mathrm{H}, \mathrm{H}_{7}, \& \mathrm{H}_{6}\right), 2.97\left(\mathrm{dd}, J_{\mathrm{H} 5, \mathrm{H} 4}=8.4 \mathrm{~Hz}, J_{\mathrm{H} 5, \mathrm{H} 6}=4.2 \mathrm{~Hz}, 1 \mathrm{H}, \mathrm{H}_{5}\right), 2.85(\mathrm{dd}$,

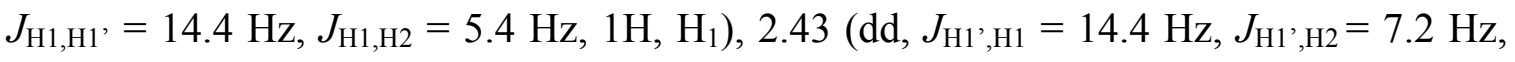
$\left.1 \mathrm{H}, \mathrm{H}_{1}{ }^{\prime}\right), 1.12-1.06\left(\mathrm{~m}, 1 \mathrm{H}, \mathrm{H}_{2}\right), 0.95\left(\mathrm{dddd}, J_{\mathrm{H} 4, \mathrm{H} 2}=17.4 \mathrm{~Hz}, J_{\mathrm{H} 4, \mathrm{H} 3}=8.4 \mathrm{~Hz}, J_{\mathrm{H} 4, \mathrm{H} 3},=\right.$ $\left.4.8 \mathrm{~Hz}, J_{\mathrm{H} 4, \mathrm{H} 5}=4.8 \mathrm{~Hz}, 1 \mathrm{H}, \mathrm{H}_{4}\right), 0.52\left(\mathrm{ddd}, J_{\mathrm{H} 3, \mathrm{H} 4}=8.4 \mathrm{~Hz}, J_{\mathrm{H} 3, \mathrm{H} 2}=4.8 \mathrm{~Hz}, J_{\mathrm{H} 3, \mathrm{H} 3}=4.8\right.$

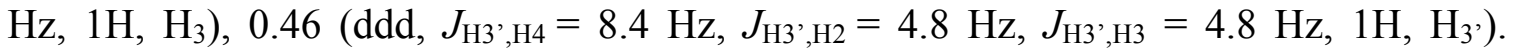
${ }^{13} \mathrm{C}\left\{{ }^{1} \mathrm{H}\right\}$ NMR (151 MHz, CD ${ }_{3} \mathrm{OD}$ ): $\delta 142.9$ (s, Ar), 129.5 (s, Ar), 129.2 (s, Ar), 126.8 (s, Ar), $76.7\left(\mathrm{~s}, \mathrm{C}_{5}\right), 76.3\left(\mathrm{~s}, \mathrm{C}_{6}\right), 64.4\left(\mathrm{~s}, \mathrm{C}_{7}\right), 40.4\left(\mathrm{~s}, \mathrm{C}_{1}\right), 22.6\left(\mathrm{~s}, \mathrm{C}_{4}\right), 19.0\left(\mathrm{~s}, \mathrm{C}_{2}\right), 10.3(\mathrm{~s}$, $\mathrm{C}_{3}$ ). HRMS $\left(\mathrm{ESI}^{+}\right)$: Calcd for $\mathrm{C}_{13} \mathrm{H}_{18} \mathrm{O}_{3} \mathrm{Na}[\mathrm{M}+\mathrm{Na}]^{+}$: 245.1148; Found: 245.1153. Specific rotation: $[\alpha]^{\mathrm{D}}{ }_{24.9}-24$. $\left(\mathrm{c}=0.44 \mathrm{CH}_{3} \mathrm{OH}\right)$.

\section{Preparation of carbocycle product 9:}

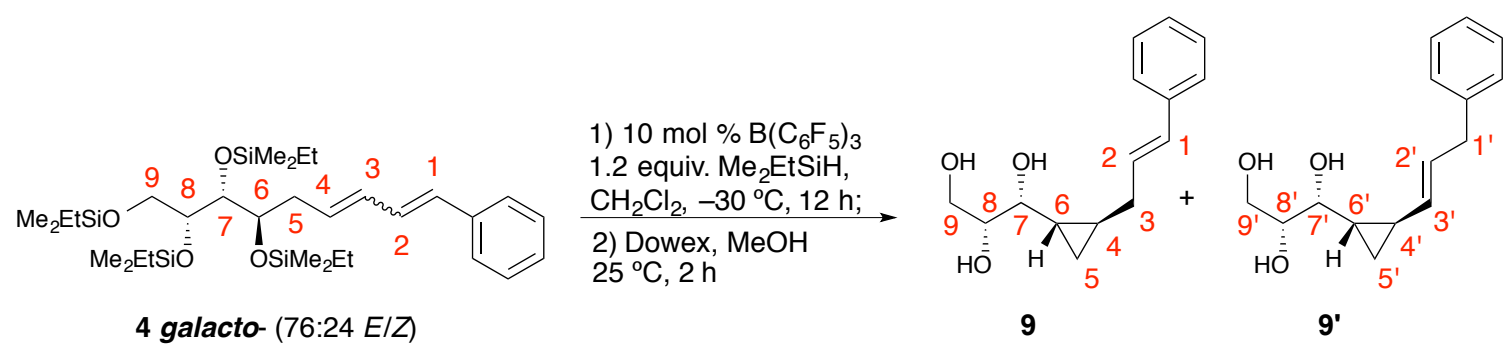

In a nitrogen-filled glovebox, a 1-dram vial was charged with $\mathrm{Me}_{2} \mathrm{EtSi}$-2-deoxygalactose diene $4(61.0 \mathrm{mg}, 0.100 \mathrm{mmol}, 1.00$ equiv. $)$ and a magnetic stir bar. A separate 1-dram vial was charged with $\mathrm{B}\left(\mathrm{C}_{6} \mathrm{~F}_{5}\right)_{3}\left(5.1 \mathrm{mg}, 0.010 \mathrm{mmol}, 0.10\right.$ equiv.) and $\mathrm{CH}_{2} \mathrm{Cl}_{2}(0.5 \mathrm{~mL})$. The vials were sealed with a septa cap and removed from the glovebox. The vials were allowed to cool to $-30{ }^{\circ} \mathrm{C}$ (dry ice/acetone bath). The $\mathrm{B}\left(\mathrm{C}_{6} \mathrm{~F}_{5}\right)_{3}$ solution was transferred to the substrate by syringe and allowed to stir as dimethylethylsilane $(15.8 \mu \mathrm{L}, 0.120 \mathrm{mmol}$, 1.2 equiv.) was added by microliter syringe. The vial was wrapped with electrical tape and transferred to a $-30{ }^{\circ} \mathrm{C}$ cryobath and allowed to stir. After 12 hours, $\mathrm{NEt}_{3}(0.05 \mathrm{~mL})$ was added by syringe through the septa and the stir bar was removed, rinsing with $\mathrm{CH}_{2} \mathrm{Cl}_{2}(2 \mathrm{~mL})$. The quenched reaction mixture was concentrated under vacuum and then loaded onto an alumina plug (neutral, $10 \% \mathrm{H}_{2} \mathrm{O} ; 2 \times 1 \mathrm{~cm}$ ) with $100 \%$ hexanes $(1.5 \mathrm{~mL}$ ) then 95:5 hexanes:diethyl ether $(1.5 \mathrm{~mL})$, eluting with 95:5 hexanes:diethyl ether $(10 \mathrm{~mL})$ into a vial. The solution was concentrated under vacuum to afford a colorless oil (67.3 $\mathrm{mg}$ ). The vial containing the silyl substrate was equipped with a stir bar and charged with Dowex ${ }^{\circledR}$ resin $(\sim 10$ beads). Methanol $(2.0 \mathrm{~mL})$ was added by syringe and the resulting solution was allowed to stir at $25^{\circ} \mathrm{C}$. After $2 \mathrm{~h}$, the solution was passed through sand $(0.1$ $\mathrm{cm})$ to remove the Dowex ${ }^{\circledR}$ beads into a tared vial, rinsing with $100 \%$ methanol. The product was purified by silica gel chromatography $(4 \times 1 \mathrm{~cm}$ column) using gradient elution $\left(100 \% \mathrm{CH}_{2} \mathrm{Cl}_{2}\right.$ to $50: 1$ to $45: 1$ to $\left.40: 1 \mathrm{CH}_{2} \mathrm{Cl}_{2}: \mathrm{MeOH}\right)$ to afford carbocycle from $\mathrm{Me}_{2}$ EtSi-2-deoxygalactose-diene 9 as a white solid $(17.6 \mathrm{mg}, 71 \%$ overall yield over 2 
steps, $0.0819 \mathrm{mmol},>98: 2 \mathrm{dr}$, and $75: 25 \mathrm{C}_{3} / \mathrm{C}_{1}$ hydride addition). Note: Stereochemistry assigned by NOE between $\mathrm{H}_{3}$ and $\mathrm{H}_{6}$.

\section{$\underline{\text { Data for } 9}$}

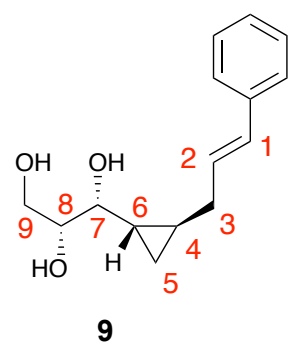

(1R,2R)-1-((1S,2S)-2-cinnamylcyclopropyl)propane-1,2,3-triol (9): ${ }^{1} \mathrm{H}$ NMR (600 $\left.\mathrm{MHz}, \mathrm{CD}_{3} \mathrm{OD}\right): \delta$ 7.37-7.33 (m, 2H, Ph), 7.28-7.21 (m, 2H, Ph), 7.18-7.11 (m, 1H, Ph), $6.48\left(\mathrm{~d}, J_{\mathrm{H} 1, \mathrm{H} 2}=16.1 \mathrm{~Hz}, 1 \mathrm{H}, \mathrm{H}_{1}\right), 6.32\left(\mathrm{dt}, J_{\mathrm{H} 2, \mathrm{H} 1}=15.9 \mathrm{~Hz}, J_{\mathrm{H} 2, \mathrm{H} 3}=6.6 \mathrm{~Hz}, 1 \mathrm{H}, \mathrm{H}_{2}\right)$, 3.74-3.67 (m, 1H, $\left.\mathrm{H}_{9}\right), 3.62-3.55\left(\mathrm{~m}, 2 \mathrm{H}, \mathrm{H}_{8} \& \mathrm{H}_{9}\right), 2.93\left(\mathrm{dd}, J_{\mathrm{H} 7, \mathrm{H} 8}=8.6 \mathrm{~Hz}, J_{\mathrm{H} 7, \mathrm{H} 6}=\right.$ $\left.4.3 \mathrm{~Hz}, 1 \mathrm{H}, \mathrm{H}_{7}\right), 2.29\left(\mathrm{dtd}, J_{\mathrm{H} 3, \mathrm{H} 3}=14.7 \mathrm{~Hz}, J_{\mathrm{H} 3, \mathrm{H} 2}=6.6 \mathrm{~Hz}, 1.5 \mathrm{~Hz}, 1 \mathrm{H}, \mathrm{H}_{3}\right), 2.11$ (dtd, $\left.J_{\mathrm{H} 3^{\prime}, \mathrm{H} 3}=15.2 \mathrm{~Hz}, J_{\mathrm{H} 3^{\prime}, \mathrm{H} 2}=6.6 \mathrm{~Hz}, J_{\mathrm{H}^{3}, \mathrm{H} 4}=1.5 \mathrm{~Hz}, 1 \mathrm{H}, \mathrm{H}^{\prime}\right), 0.97-0.85\left(\mathrm{~m}, 2 \mathrm{H}, \mathrm{H}_{4} \& \mathrm{H}_{6}\right)$,

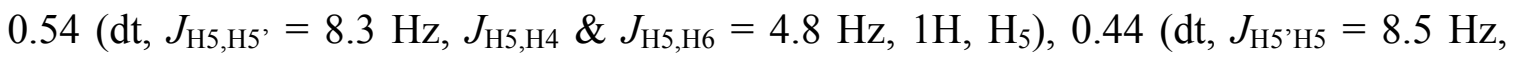
$\left.J_{\mathrm{H} 5^{\prime}, \mathrm{H} 4} \& J_{\mathrm{H} 5^{\prime}, \mathrm{H} 6}=4.9 \mathrm{~Hz}, 1 \mathrm{H}, \mathrm{H}_{5}\right) .{ }^{13} \mathrm{C}\left\{{ }^{1} \mathrm{H}\right\} \mathrm{NMR}\left(151 \mathrm{MHz}, \mathrm{CD}_{3} \mathrm{OD}\right): \delta 139.3(\mathrm{~s}, \mathrm{Ph})$, $130.5\left(\mathrm{~s}, \mathrm{C}_{1}\right), 129.4\left(\mathrm{~s}, \mathrm{C}_{2}\right), 128.3(\mathrm{~s}, \mathrm{Ph}), 127.0$ (s, Ph), 126.9 (s, Ph), 76.9 (s, $\left.\mathrm{C}_{8}\right), 76.4$ (s, $\left.\mathrm{C}_{7}\right), 64.5\left(\mathrm{~s}, \mathrm{C}_{9}\right), 37.9\left(\mathrm{~s}, \mathrm{C}_{3}\right), 22.5\left(\mathrm{~s}, \mathrm{C}_{6}\right), 17.6\left(\mathrm{~s}, \mathrm{C}_{4}\right), 10.1\left(\mathrm{~s}, \mathrm{C}_{5}\right)$. HRMS (ESI $\left.{ }^{+}\right)$: Calcd for $\mathrm{C}_{15} \mathrm{H}_{20} \mathrm{O}_{3} \mathrm{Na}[\mathrm{M}+\mathrm{Na}]^{+}: 241.1199$; Found: 241.1204. Specific rotation: $[\alpha]_{25.8}^{\mathrm{D}}-10.3$ $\left(\mathrm{c}=1.05 \mathrm{CH}_{3} \mathrm{OH}\right)$.

\section{Data for 9'}

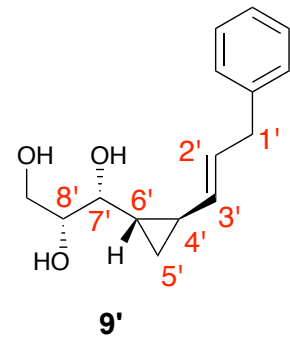

(1R,2R)-1-((1S,2R)-2-((E)-3-phenylprop-1-en-1-yl)cyclopropyl)propane-1,2,3-triol (9'): ${ }^{1} \mathrm{H}$ NMR (600 MHz, $\left.\mathrm{CD}_{3} \mathrm{OD}\right): \delta$ 7.28-7.21 (m, 2H, Ph), 7.18-7.11 (m, 3H, Ph), 5.64 $\left(\mathrm{dt}, J_{\mathrm{H} 2^{\prime}, \mathrm{H} 3^{\prime}}=15.1 \mathrm{~Hz}, J_{\mathrm{H}^{2}, \mathrm{H}^{\prime}}=6.9 \mathrm{~Hz}, 1 \mathrm{H}, \mathrm{H}_{2}{ }^{\prime}\right), 5.16\left(\mathrm{ddt}, J_{\mathrm{H} 3^{\prime}, \mathrm{H}{ }^{\prime}}=15.1 \mathrm{~Hz}, J_{\mathrm{H} 3^{\prime}, \mathrm{H} 4^{\prime}}=\right.$ $\left.8.3 \mathrm{~Hz}, J_{\mathrm{H}^{3}, \mathrm{H} 1}{ }^{\prime}=1.5 \mathrm{~Hz}, 1 \mathrm{H}, \mathrm{H}_{3}{ }^{\prime}\right), 3.74-3.67\left(\mathrm{~m}, 1 \mathrm{H}, \mathrm{H}_{9}\right), 3.62-3.55\left(\mathrm{~m}, 2 \mathrm{H}, \mathrm{H}_{9}\right.$, \& $\left.\mathrm{H}_{8}\right)$, $3.29\left(\mathrm{~d}, J_{\mathrm{H}^{\prime}, \mathrm{H} 2^{\prime}}=6.9 \mathrm{~Hz}, 1 \mathrm{H}, \mathrm{H}^{\prime}\right), 2.98\left(\mathrm{dd}, J_{\mathrm{H}^{\prime}, \mathrm{H} 6^{\prime}}=8.6 \mathrm{~Hz}, J_{\mathrm{H}^{\prime}, \mathrm{H} 8^{\prime}}=4.1 \mathrm{~Hz}, 1 \mathrm{H}, \mathrm{H}_{7^{\prime}}\right)$, $1.45\left(\mathrm{tt}, J_{\mathrm{H}^{\prime}, \mathrm{H}^{\prime}}=J_{\mathrm{H}^{\prime}, \mathrm{H}^{\prime}}=8.7 \mathrm{~Hz}, J_{\mathrm{H}^{\prime}, \mathrm{H}^{\prime \prime}}=J_{\mathrm{H}^{\prime}{ }^{\prime} 4^{\prime}}=4.5 \mathrm{~Hz}, 1 \mathrm{H}, \mathrm{H}_{6}{ }^{\circ}\right), 1.03\left(\mathrm{tt}, J_{\mathrm{H} 4^{\prime}, \mathrm{H} 3^{\prime}}=\right.$ $\left.J_{\mathrm{H}^{\prime}, \mathrm{H} 5^{\prime}}=8.5 \mathrm{~Hz}, J_{\mathrm{H}^{\prime}, \mathrm{H} 6^{\prime}}=J_{\mathrm{H}^{4}, \mathrm{H} 5^{\prime}}=4.9 \mathrm{~Hz}, 1 \mathrm{H}, \mathrm{H}^{\prime}\right), 0.67\left(\mathrm{dt}, J_{\mathrm{H}^{\prime}, \mathrm{H} 5^{\prime}}=8.5 \mathrm{~Hz}, J_{\mathrm{H} 5^{\prime}, \mathrm{H} 4^{\prime}} \&\right.$ $\left.J_{\mathrm{H} 5^{\prime}, \mathrm{H} 6^{\prime}}=5.0 \mathrm{~Hz}, 1 \mathrm{H}, \mathrm{H}_{5}{ }^{\prime}\right), 0.57\left(\mathrm{dt}, J_{\mathrm{H} 5^{\prime}, \mathrm{H} 5^{\prime}}=8.6 \mathrm{~Hz}, J_{\mathrm{H} 5^{\prime}, \mathrm{H} 4^{\prime}} \& J_{\mathrm{H}^{\prime}, \mathrm{H} 6^{\prime}}=5.0 \mathrm{~Hz}, 1 \mathrm{H}\right.$, 
$\mathrm{H}_{5}$ "). ${ }^{13} \mathrm{C}\left\{{ }^{1} \mathrm{H}\right\}$ NMR (151 MHz, $\left.\mathrm{CD}_{3} \mathrm{OD}\right): \delta 142.3(\mathrm{~s}, \mathrm{Ph}), 135.1(\mathrm{~s}, \mathrm{Ph}), 131.3\left(\mathrm{~s}, \mathrm{C}_{3}{ }^{\prime}\right)$, $129.5(\mathrm{~s}, \mathrm{Ph}), 129.3\left(\mathrm{~s}, \mathrm{C}_{2}{ }^{\prime}\right), 127.8(\mathrm{~s}, \mathrm{Ph}), 76.3\left(\mathrm{~s}, \mathrm{C}_{8^{\prime}}\right), 76.2,64.4\left(\mathrm{~s}, \mathrm{C}_{7}{ }^{\prime}\right), 39.8\left(\mathrm{~s}, \mathrm{C}_{1}{ }^{\prime}\right)$, $24.3\left(\mathrm{~s}, \mathrm{C}_{6}\right), 20.4\left(\mathrm{~s}, \mathrm{C}_{4}\right), 11.6\left(\mathrm{~s}, \mathrm{C}_{5}\right)$.

\section{Preparation of cyclopropane product 10:}

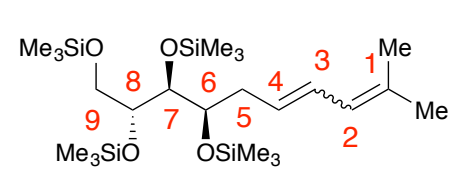

5 galacto- $(68: 32 \mathrm{E} / Z)$
1) $10 \mathrm{~mol} \% \mathrm{~B}\left(\mathrm{C}_{6} \mathrm{~F}_{5}\right)_{3}$ 1.1 equiv. $\mathrm{Ph}_{3} \mathrm{SiH}$, 2) Dowex, $\mathrm{MeOH}$ $25^{\circ} \mathrm{C}, 2 \mathrm{~h}$
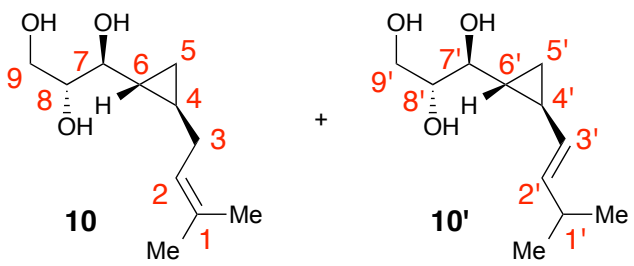

In a nitrogen-filled glovebox, a 1-dram vial was charged with $\mathrm{B}\left(\mathrm{C}_{6} \mathrm{~F}_{5}\right)_{3}(5.1 \mathrm{mg}, 0.010$ mmol, 0.10 equiv.) then triphenylsilane (28.8 mg, $0.110 \mathrm{mmol}, 1.1$ equiv.), then $\mathrm{Me}_{3} \mathrm{Si}-$ styryl 2-deoxygalactose 5 (50.5 mg, $0.100 \mathrm{mmol}, 1.00$ equiv.) and a magnetic stir bar. By syringe $\mathrm{CH}_{2} \mathrm{Cl}_{2}(0.5 \mathrm{~mL})$ was added. The vial was sealed with a septa cap and removed from the glovebox and allowed to stir at $22{ }^{\circ} \mathrm{C}$. After 2 hours, $\mathrm{NEt}_{3}(0.05 \mathrm{~mL})$ was added by syringe through the septa and the stir bar was removed, rinsing with $\mathrm{CH}_{2} \mathrm{Cl}_{2}(2 \mathrm{~mL})$. The quenched reaction mixture was concentrated under vacuum and then loaded onto an alumina plug (neutral, $10 \% \mathrm{H}_{2} \mathrm{O} ; 2 \times 1 \mathrm{~cm}$ ) with $100 \%$ hexanes $(1.5 \mathrm{~mL})$ then $95: 5$ hexanes:diethyl ether $(1.5 \mathrm{~mL})$, eluting with 95:5 hexanes:diethyl ether $(10 \mathrm{~mL})$ into a tared vial. The solution was concentrated under vacuum to afford a colorless oil (70.7 $\mathrm{mg}$ ). The vial containing the silyl substrate was equipped with a stir bar and charged with Dowex $^{\circledR}$ resin $(\sim 10$ beads). Methanol $(2.0 \mathrm{~mL})$ was added by syringe and the resulting solution was allowed to stir at $25^{\circ} \mathrm{C}$. After $2 \mathrm{~h}$, the solution was passed through sand $(0.1$ $\mathrm{cm})$ to remove the Dowex ${ }^{\circledR}$ beads into a tared vial, rinsing with $100 \%$ methanol. The product was purified by silica gel chromatography (4 x $1 \mathrm{~cm}$ column) using gradient elution (100\% $\mathrm{CH}_{2} \mathrm{Cl}_{2}$ to $50: 1$ to $45: 1$ to $40: 1$ to $35: 1$ to $30: 1$ to $\left.25: 1 \mathrm{CH}_{2} \mathrm{Cl}_{2}: \mathrm{MeOH}\right)$ to afford cyclopropane from $\mathrm{Me}_{3} \mathrm{Si}$-2-deoxygalactose-diene $\mathbf{1 0}$ as a colorless oil $(22.9 \mathrm{mg}$, 95\% overall yield over 2 steps, $0.103 \mathrm{mmol},>98: 2 \mathrm{dr}, 83: 17 \mathrm{C}_{3} / \mathrm{C}_{1}$ hydride addition). Note: Stereochemistry assigned by NOE between $\mathrm{H}_{3}$ and $\mathrm{H}_{6}$.

\section{Data for 10}

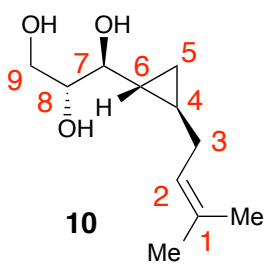

(1S,2R)-1-((1R,2R)-2-(3-methylbut-2-en-1-yl)cyclopropyl)propane-1,2,3-triol (10): ${ }^{1} \mathrm{H}$ NMR $\left(600 \mathrm{MHz}, \mathrm{CD}_{3} \mathrm{OD}\right): \delta 5.25$ (tsept, $\left.J_{\mathrm{H} 2, \mathrm{H} 3}=7.1 \mathrm{~Hz}, J_{\mathrm{H} 2, \mathrm{Me}}=1.5 \mathrm{~Hz}, 1 \mathrm{H}_{1} \mathrm{H}_{2}\right), 3.74$ 
$3.69\left(\mathrm{~m}, 1 \mathrm{H}, \mathrm{H}_{9}\right), 3.62-3.57\left(\mathrm{~m}, 2 \mathrm{H}, \mathrm{H}_{9}, \& \mathrm{H}_{8}\right), 2.90\left(\mathrm{dd}, J=8.1,4.1 \mathrm{~Hz}, 1 \mathrm{H}, \mathrm{H}_{7}\right), 2.15$ $\left(\mathrm{dt}, J_{\mathrm{H} 3, \mathrm{H} 3},=14.0 \mathrm{~Hz}, J_{\mathrm{H} 3, \mathrm{H} 2}=7.0 \mathrm{~Hz}, 1 \mathrm{H}, \mathrm{H}_{3}\right), 1.92\left(\mathrm{dt}, J_{\mathrm{H} 3^{\prime}, \mathrm{H} 3}=14.0 \mathrm{~Hz}, J_{\mathrm{H} 3^{\prime}, \mathrm{H} 2}=7.0\right.$ $\left.\mathrm{Hz}, 1 \mathrm{H}, \mathrm{H}_{3}\right), 1.72\left(\mathrm{~d}, J_{\mathrm{Me}, \mathrm{H} 2}=1.4 \mathrm{~Hz}, 3 \mathrm{H}, \mathrm{H}_{\mathrm{Me}}\right), 1.63\left(\mathrm{~d}, J_{\mathrm{Me}, \mathrm{H} 2}=1.3 \mathrm{~Hz}, 3 \mathrm{H}, \mathrm{H}_{\mathrm{Me}}\right), 0.85-$ $0.77\left(\mathrm{~m}, 2 \mathrm{H}, \mathrm{H}_{6} \& \mathrm{H}_{4}\right), 0.43\left(\mathrm{dt}, J_{\mathrm{H} 5, \mathrm{H} 5}=8.0 \mathrm{~Hz}, J_{\mathrm{H} 5, \mathrm{H} 4} \& J_{\mathrm{H} 5, \mathrm{H} 7}=4.8 \mathrm{~Hz}, 1 \mathrm{H}, \mathrm{H}_{5}\right), 0.34$ $\left(\mathrm{dt}, J_{\mathrm{H} 5^{\prime}, \mathrm{H} 5}=8.0 \mathrm{~Hz}, J_{\mathrm{H} 5^{\prime}, \mathrm{H} 4} \& J_{\mathrm{H} 5^{\prime}, \mathrm{H} 7}=4.8 \mathrm{~Hz}, 1 \mathrm{H}, \mathrm{H}_{5^{\prime}}\right) .{ }^{13} \mathrm{C}\left\{{ }^{1} \mathrm{H}\right\} \mathrm{NMR}(151 \mathrm{MHz}$, $\mathrm{CD}_{3} \mathrm{OD}$ ): $\delta 132.4\left(\mathrm{~s}, \mathrm{C}_{1}\right), 124.5\left(\mathrm{~s}, \mathrm{C}_{2}\right), 76.8\left(\mathrm{~s}, \mathrm{C}_{8}\right), 76.4\left(\mathrm{~s}, \mathrm{C}_{7}\right), 64.4\left(\mathrm{~s}, \mathrm{C}_{9}\right), 32.7\left(\mathrm{~s}, \mathrm{C}_{3}\right)$, $25.9\left(\mathrm{~s}, \mathrm{C}_{\mathrm{Me}}\right), 22.1\left(\mathrm{~s}, \mathrm{C}_{4}\right.$ or $\left.\mathrm{C}_{6}\right), 18.1\left(\mathrm{~s}, \mathrm{C}_{6}\right.$ or $\left.\mathrm{C}_{4}\right), 17.9\left(\mathrm{~s}, \mathrm{C}_{\mathrm{Me}}\right), 9.7\left(\mathrm{~s}, \mathrm{C}_{5}\right)$. HRMS $\left(\mathrm{ESI}^{+}\right)$: Calcd for $\mathrm{C}_{11} \mathrm{H}_{20} \mathrm{O}_{3} \mathrm{Na}[\mathrm{M}+\mathrm{H}]^{+}:$223.1310; Found: 223.1303. Specific rotation: $[\alpha]_{24.6}^{\mathrm{D}}+24 .\left(\mathrm{c}=0.24 \mathrm{CH}_{3} \mathrm{OH}\right)$.

\section{Data for 10'}

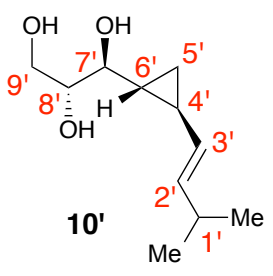

\section{(1S,2R)-1-((1R,2S)-2-((E)-3-methylbut-1-en-1-yl)cyclopropyl)propane-1,2,3-triol} (10'): ${ }^{1} \mathrm{H}$ NMR $\left(600 \mathrm{MHz}, \mathrm{CD}_{3} \mathrm{OD}\right): \delta 5.46\left(\mathrm{dd}, J_{\mathrm{H} 2^{\prime}, \mathrm{H} 3^{\prime}}=15.3 \mathrm{~Hz}, J_{\mathrm{H}^{\prime}, \mathrm{H} 1}{ }^{\prime}=6.8 \mathrm{~Hz}, 1 \mathrm{H}\right.$, $\left.\mathrm{H}_{2}{ }^{\prime}\right), 5.03\left(\mathrm{ddd}, J_{\mathrm{H}^{\prime}, \mathrm{H}^{\prime}}=15.3 \mathrm{~Hz}, J_{\mathrm{H}^{\prime}, \mathrm{H} 4^{\prime}}=8.3 \mathrm{~Hz}, J_{\mathrm{H}^{\prime}, \mathrm{H}^{\prime}}=1.3 \mathrm{~Hz}, 1 \mathrm{H}, \mathrm{H}_{3^{\prime}}\right), 3.62-3.57$ (m, 3H, $\left.\mathrm{H}_{9}, \& \mathrm{H}_{8}{ }^{\prime}\right), 2.98-2.95\left(\mathrm{~m}, 1 \mathrm{H}, \mathrm{H}_{7^{\prime}}\right), 2.24-2.18\left(\mathrm{~m}, 1 \mathrm{H}, \mathrm{H}_{1^{\prime}}\right), 2.15\left(\mathrm{~m}, 1 \mathrm{H}, \mathrm{H}_{6}\right)$, $1.40\left(\mathrm{dq}, J_{\mathrm{H}^{4}, \mathrm{H} 3^{\prime}}=8.4 \mathrm{~Hz}, J_{\mathrm{H}^{\prime}, \mathrm{H} 5^{\prime}}=4.3 \mathrm{~Hz}, 1 \mathrm{H}, \mathrm{H}_{4}{ }^{\prime}\right), 0.96\left(\mathrm{~s}, 3 \mathrm{H}, \mathrm{H}_{\mathrm{Me}}\right), 0.95(\mathrm{~s}, 3 \mathrm{H}$, $\mathrm{H}_{\mathrm{Me}}$ ), 0.64 (app dt, $J_{\mathrm{H} 5^{\prime}, \mathrm{H} 5^{\prime}}=8.5 \mathrm{~Hz}, J_{\mathrm{H} 5^{\prime}, \mathrm{H} 4^{\prime}} \& J_{\mathrm{H}^{\prime}, \mathrm{H} 6^{\prime}}=5.0 \mathrm{~Hz}, 1 \mathrm{H}, \mathrm{H}_{5^{\prime}}$ ), 0.53 (app dt,

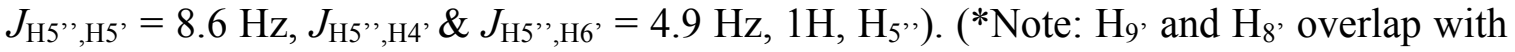
$\mathrm{H}_{9}$ and $\mathrm{H}_{8}$. Also $\mathrm{H}_{6}$, overlaps with $\mathrm{H}_{3}$ ). ${ }^{13} \mathrm{C}\left\{{ }^{1} \mathrm{H}\right\}$ NMR (151 MHz, $\mathrm{CD}_{3} \mathrm{OD}$ ): $\delta 136.6$ (s, $\left.\mathrm{C}_{2}{ }^{\prime}\right), 130.9\left(\mathrm{~s}, \mathrm{C}_{3^{\prime}}\right), 76.4\left(\mathrm{~s}, \mathrm{C}_{8^{\prime}}\right), 76.2\left(\mathrm{~s}, \mathrm{C}_{7^{\prime}}\right), 64.4\left(\mathrm{~s}, \mathrm{C}_{9}\right), 32.2\left(\mathrm{~s}, \mathrm{C}_{1}\right), 23.1\left(\mathrm{~s}, \mathrm{C}_{\mathrm{Me}}\right)$, $23.1\left(\mathrm{~s}, \mathrm{C}_{6}\right)\left(\mathrm{s}, \mathrm{C}_{\mathrm{Me}}\right), 20.4\left(\mathrm{~s}, \mathrm{C}_{4}\right), 11.5\left(\mathrm{~s}, \mathrm{C}_{5}\right)$.

\section{Preparation of cyclic product 11:}

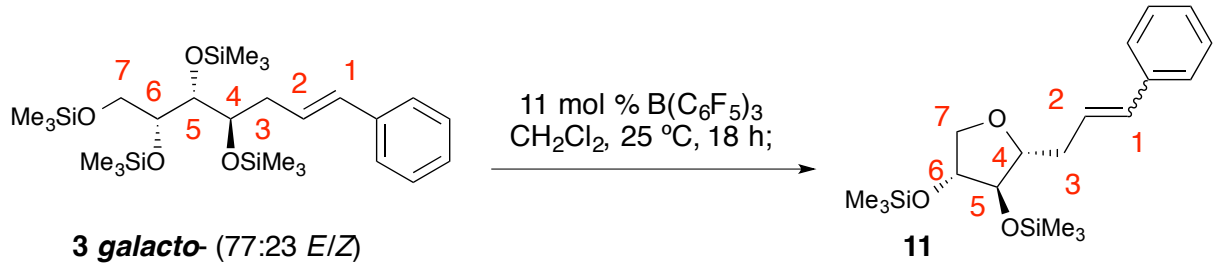

In a nitrogen-filled glovebox, a 1-dram vial was charged with $\mathrm{B}\left(\mathrm{C}_{6} \mathrm{~F}_{5}\right)_{3}(5.1 \mathrm{mg}, 0.010$ mmol, 0.10 equiv.) then TMS-styryl 2-deoxygalactose $3(52.2 \mathrm{mg}, 0.0990 \mathrm{mmol}, 1.00$ equiv.) and a stir bar. At $25{ }^{\circ} \mathrm{C}, \mathrm{CH}_{2} \mathrm{Cl}_{2}(0.5 \mathrm{~mL})$ was added by syringe. The vial was sealed with a septa cap and removed from the glovebox. The vial was wrapped with electrical tape and allowed to stir at $22{ }^{\circ} \mathrm{C}$. After 18 hours, $\mathrm{NEt}_{3}(0.05 \mathrm{~mL})$ was added by 
syringe through the septa and the stir bar was removed, rinsing with $\mathrm{CH}_{2} \mathrm{Cl}_{2}(0.2 \mathrm{~mL})$. The quenched reaction mixture was concentrated under vacuum and then loaded onto an alumina plug (neutral, $10 \% \mathrm{H}_{2} \mathrm{O} ; 2 \times 1 \mathrm{~cm}$ ) with $100 \%$ hexanes $(1.5 \mathrm{~mL})$ then 95:5 hexanes:diethyl ether $(1.5 \mathrm{~mL})$, eluting with $95: 5$ hexanes:diethyl ether $(10 \mathrm{~mL})$ into a tared vial. The solution was concentrated under vacuum to afford a colorless oil (42.2 $\mathrm{mg})$. The product was purified by alumina chromatography $\left(10 \% \mathrm{H}_{2} \mathrm{O}, 5 \times 1 \mathrm{~cm}\right.$ column$)$ using gradient elution (100\% hexanes to $20: 1$ to $2: 1$ hexanes: $\left.\mathrm{Et}_{2} \mathrm{O}\right)$ to afford cyclized TMS-styryl 2-deoxygalactose 11 as a colorless oil $(27.9 \mathrm{mg}, 77 \%$ overall yield over 2 steps, $0.0765 \mathrm{mmol}, 74: 26 \mathrm{E} / \mathrm{Z})$.

\section{Data for 11}

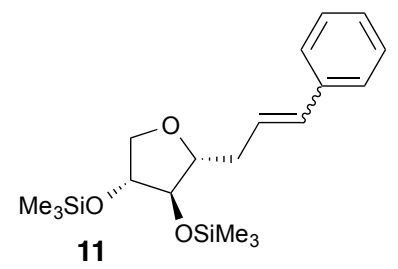

(((2R,3R,4R)-2-(3-phenylallyl)tetrahydrofuran-3,4-diyl)bis(oxy))bis(trimethylsilane) (11): ${ }^{1} \mathrm{H} \mathrm{NMR}\left(600 \mathrm{MHz}, \mathrm{CDCl}_{3}\right): \delta 7.36$ (d, $\left.J=7.8 \mathrm{~Hz}, 2 \mathrm{H}, \mathrm{Ar}\right), 7.32-7.27$ (m, 6H, Ar), $7.23-7.18(\mathrm{~m}, 2 \mathrm{H}, \mathrm{Ar}), 6.55\left(\mathrm{~d}, J_{\mathrm{H} 1, \mathrm{H} 2}=11.4 \mathrm{~Hz}, 1 \mathrm{H}, \mathrm{H}_{1}, Z\right), 6.47\left(\mathrm{~d}, J_{\mathrm{H} 1, \mathrm{H} 2}=15.6 \mathrm{~Hz}\right.$, $\left.1 \mathrm{H}, \mathrm{H}_{1}, E\right), 6.26\left(\mathrm{dt}, J_{\mathrm{H} 2, \mathrm{H} 1}=16.2 \mathrm{~Hz}, J_{\mathrm{H} 2, \mathrm{H} 3}=7.2 \mathrm{~Hz}, 1 \mathrm{H}, \mathrm{H}_{2}, E\right), 5.75\left(\mathrm{dt}, J_{\mathrm{H} 2, \mathrm{H} 1}=11.4\right.$ $\left.\mathrm{Hz}, J_{\mathrm{H} 2, \mathrm{H} 3}=7.2 \mathrm{~Hz}, 1 \mathrm{H}, \mathrm{H}_{2}, Z\right), 4.08\left(\mathrm{dt}, J_{\mathrm{H} 6, \mathrm{H} 7}=4.2 \mathrm{~Hz}, J_{\mathrm{H} 6, \mathrm{H} 5}=3.0 \mathrm{~Hz}, 1 \mathrm{H}, \mathrm{H}_{6}, E\right)$, $4.05\left(\mathrm{dt}, J_{\mathrm{H} 6, \mathrm{H} 7}=4.8 \mathrm{~Hz}, J_{\mathrm{H} 6, \mathrm{H} 5}=3.0 \mathrm{~Hz}, 1 \mathrm{H}, \mathrm{H}_{6}, Z\right), 3.96\left(\mathrm{dd}, J_{\mathrm{H} 7, \mathrm{H} 7}=9.6 \mathrm{~Hz}, J_{\mathrm{H} 7, \mathrm{H} 6}=\right.$ $\left.4.8 \mathrm{~Hz}, 1 \mathrm{H}, \mathrm{H}_{7}, E\right), 3.95\left(\mathrm{dd}, J_{\mathrm{H} 7, \mathrm{H} 7}=9.6 \mathrm{~Hz}, J_{\mathrm{H} 7, \mathrm{H} 6}=4.8 \mathrm{~Hz}, 1 \mathrm{H}, \mathrm{H}_{7}, Z\right), 3.87$ (app t, $J=$ $\left.3.0 \mathrm{~Hz}, 1 \mathrm{H}, \mathrm{H}_{5}, E\right), 3.79$ (app t, $\left.J=4.2 \mathrm{~Hz}, 1 \mathrm{H}, \mathrm{H}_{5}, Z\right), 3.79-3.75\left(\mathrm{~m}, 3 \mathrm{H}, \mathrm{H}_{4}(E), \mathrm{H}_{4}(Z)\right.$, $\left.\& \mathrm{H}_{7}(Z)\right), 3.72\left(\mathrm{dd}, J_{\mathrm{H} 7^{\prime}, \mathrm{H} 7}=10.2 \mathrm{~Hz}, J_{\mathrm{H} 7^{\prime}, \mathrm{H} 6}=3.0 \mathrm{~Hz}, 1 \mathrm{H}, \mathrm{H}_{7}, E\right), 2.74-2.69\left(\mathrm{~m}, 1 \mathrm{H}, \mathrm{H}_{3}\right.$, Z), 2.64-2.62 (m, 1H, H $\left.{ }_{3}, Z\right), 2.59-2.50$ (m, 2H, $\left.\mathrm{H}_{3} \& \mathrm{H}_{3}, E\right), 0.133$ (s, 9H, - $\left.\mathrm{SiCH}_{3}, E\right)$, $0.127\left(\mathrm{~s}, 9 \mathrm{H},-\mathrm{SiCH}_{3}, E\right), 0.10\left(\mathrm{~s}, 9 \mathrm{H},-\mathrm{SiCH}_{3}, Z\right), 0.07\left(\mathrm{~s}, 9 \mathrm{H},-\mathrm{SiCH}_{3}, Z\right) .{ }^{13} \mathrm{C}\left\{{ }^{1} \mathrm{H}\right\} \mathrm{NMR}$ (151 MHz, $\mathrm{CDCl}_{3}$ ): $\delta 137.7$ (s, Ar, E), 137.5 (s, Ar, Z), 132.3 (s, C $\left.1, E\right), 130.9$ (s, C 1 , Z), 129.0 (s, Ar, Z), 128.6 (s, Ar, E), 128.4 (s, Ar, Z), 128.3, (s, C $2, Z$ ), 127.2 (s, Ar, E), 126.8 (s, Ar, Z), 126.7 (s, $\left.\mathrm{C}_{2}, E\right), 126.2$ (s, Ar, E), 85.1 (s, $\left.\mathrm{C}_{4}, Z\right), 84.9$ (s, $\left.\mathrm{C}_{4}, E\right), 82.2\left(\mathrm{~s}, \mathrm{C}_{5}, Z\right)$, 82.1 (s, $\left.\mathrm{C}_{5}, E\right), 79.0\left(\mathrm{~s}, \mathrm{C}_{6}, E\right), 78.8$ (s, $\left.\mathrm{C}_{6}, Z\right), 73.54$ (s, $\left.\mathrm{C}_{7}, Z\right), 73.50$ (s, $\left.\mathrm{C}_{7}, E\right)$, 0.5, 0.4, $0.2,0.1$ (each a s, $\left.-\mathrm{Si}\left(\mathrm{CH}_{3}\right)_{3}\right)$.

\section{Procedure for preparation of $1^{\circ}$ bond reduction from 11}




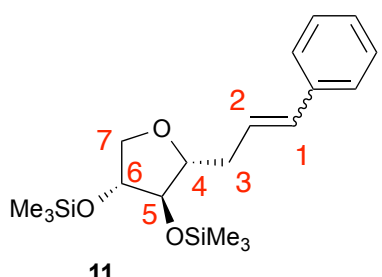

11
1) $15 \mathrm{~mol} \% \mathrm{~B}\left(\mathrm{C}_{6} \mathrm{~F}_{5}\right)_{3}$
1.5 equiv. $\mathrm{Et}_{3} \mathrm{SiH}$,
$\mathrm{CH}_{2} \mathrm{Cl}_{2}, 22{ }^{\circ} \mathrm{C}, 1 \mathrm{~h}$;
2) Dowex, $\mathrm{MeOH}$ $22{ }^{\circ} \mathrm{C}, 2 \mathrm{~h}$

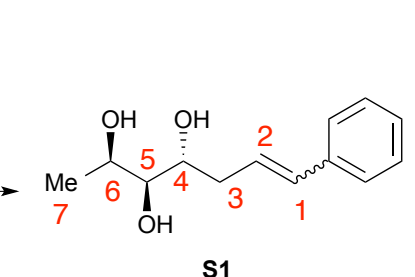

s1

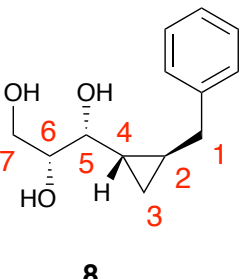

8

In a nitrogen-filled glovebox, a 1-dram vial was charged with 11 (27.9 $\mathrm{mg}, 0.0765 \mathrm{mmol}$, 1.00 equiv.), $\mathrm{B}\left(\mathrm{C}_{6} \mathrm{~F}_{5}\right)_{3}\left(7.1 \mathrm{mg}, 0.015 \mathrm{mmol}, 0.20\right.$ equiv.) and a stir bar. At $25{ }^{\circ} \mathrm{C}, \mathrm{CH}_{2} \mathrm{Cl}_{2}$ $(0.5 \mathrm{~mL})$ was added by syringe and triethylsilane $(18.0 \mu \mathrm{L}, 0.113 \mathrm{mmol}, 1.50$ equiv. $)$ was added by microliter syringe. The vial was sealed with a septa cap and removed from the glovebox. The vial was wrapped with electrical tape and allowed to stir at $22{ }^{\circ} \mathrm{C}$. After 1 hour, $\mathrm{NEt}_{3}(0.05 \mathrm{~mL})$ was added by syringe through the septa and the stir bar was removed, rinsing with $\mathrm{CH}_{2} \mathrm{Cl}_{2}(0.2 \mathrm{~mL})$. The quenched reaction mixture was concentrated under vacuum and then loaded onto an alumina plug (neutral, $10 \% \mathrm{H}_{2} \mathrm{O} ; 2 \times 1 \mathrm{~cm}$ ) with 100\% hexanes $(1.5 \mathrm{~mL})$ then 95:5 hexanes:diethyl ether $(1.5 \mathrm{~mL})$, eluting with 95:5 hexanes:diethyl ether $(10 \mathrm{~mL})$ into a tared vial. The solution was concentrated under vacuum to afford a colorless oil $(47.3 \mathrm{mg})$. The vial containing the silyl substrate was equipped with a stir bar and charged with Dowex ${ }^{\circledR}$ resin $(\sim 10$ beads). Methanol $(2.0 \mathrm{~mL})$ was added by syringe and the resulting solution was allowed to stir at $25^{\circ} \mathrm{C}$. After $2 \mathrm{~h}$, the solution was passed through sand $(0.1 \mathrm{~cm})$ to remove the Dowex ${ }^{\circledR}$ beads into a tared vial, rinsing with $100 \%$ methanol. The product was purified by silica gel chromatography (5 x $1 \mathrm{~cm}$ column) using gradient elution $\left(100 \% \mathrm{CH}_{2} \mathrm{Cl}_{2}\right.$ to $50: 1$ to $40: 1$ to $30: 1$ to 20:1 $\left.\mathrm{CH}_{2} \mathrm{Cl}_{2}: \mathrm{MeOH}\right)$ to afford primary reduced 2-deoxy galactose $\mathbf{S 1}$ as a colorless oil (7.1 $\mathrm{mg}, 42 \%$ overall yield over 2 steps, $0.032 \mathrm{mmol}, 51: 49 \mathrm{E} / \mathrm{Z}$ ). [ ${ }^{*}$ Note: Remaining material is cyclopropyl product 8 ( $9.4 \mathrm{mg}, 55 \%$ overall yield over 2 steps, $0.042 \mathrm{mmol})$.]

\section{Data for S1:}

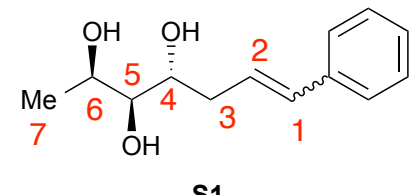

s1

(2R,3S,4R)-7-phenylhept-6-ene-2,3,4-triol (S1): Characterized as a 51:49 E/Z mixture. ${ }^{1} \mathrm{H}$ NMR (600 MHz, CD $\left.{ }_{3} \mathrm{OD}\right): \delta 7.38(\mathrm{~d}, J=7.2 \mathrm{~Hz}, 2 \mathrm{H}, \mathrm{Ph}), 7.35-7.31(\mathrm{~m}, 4 \mathrm{H}, \mathrm{Ph})$, $7.28(\mathrm{t}, J=7.2 \mathrm{~Hz}, 2 \mathrm{H}, \mathrm{Ph}), 7.22-7.17(\mathrm{~m}, 2 \mathrm{H}, \mathrm{Ph}), 6.55\left(\mathrm{~d}, J_{\mathrm{H} 1, \mathrm{H} 2}=11.4 \mathrm{~Hz}, 1 \mathrm{H}, \mathrm{H}_{1}, \mathrm{Z}\right)$, $6.51\left(\mathrm{~d}, J_{\mathrm{H} 1, \mathrm{H} 2}=15.6 \mathrm{~Hz}, 1 \mathrm{H}, \mathrm{H}_{1}, E\right), 6.40\left(\mathrm{ddd}, J_{\mathrm{H} 2, \mathrm{H} 1}=15.6 \mathrm{~Hz}, J_{\mathrm{H} 2, \mathrm{H} 3}=7.2 \mathrm{~Hz}, J_{\mathrm{H} 2, \mathrm{H} 3}\right.$ $\left.=6.6 \mathrm{~Hz}, 1 \mathrm{H}, \mathrm{H}_{2}, E\right), 5.88\left(\mathrm{dt}, J_{\mathrm{H} 2, \mathrm{H} 1}=12.0 \mathrm{~Hz}, J_{\mathrm{H} 2, \mathrm{H} 3}=7.2 \mathrm{~Hz}, 1 \mathrm{H}, \mathrm{H}_{2}, Z\right), 4.06(\mathrm{dq}$, $\left.J_{\mathrm{H} 6, \mathrm{H} 7}=6.6 \mathrm{~Hz}, J_{\mathrm{H} 6, \mathrm{H} 5}=2.4 \mathrm{~Hz}, 1 \mathrm{H}, \mathrm{H}_{6}, \mathrm{E}\right), 4.00\left(\mathrm{dq}, J_{\mathrm{H} 6, \mathrm{H} 7}=6.6 \mathrm{~Hz}, J_{\mathrm{H} 6, \mathrm{H} 5}=3.0 \mathrm{~Hz}, 1 \mathrm{H}\right.$, $\left.\mathrm{H}_{6}, Z\right), 3.76\left(\mathrm{dt}, J_{\mathrm{H} 4, \mathrm{H} 5}=7.8 \mathrm{~Hz}, J_{\mathrm{H} 4, \mathrm{H} 3}=3.0 \mathrm{~Hz}, 1 \mathrm{H}, \mathrm{H}_{4}, E\right), 3.75-3.73\left(\mathrm{~m}, 1 \mathrm{H}, \mathrm{H}_{4}, Z\right)$, $3.21\left(\mathrm{dd}, J_{\mathrm{H} 5, \mathrm{H} 4}=7.8 \mathrm{~Hz}, J_{\mathrm{H} 5, \mathrm{H} 4}=2.4 \mathrm{~Hz}, 1 \mathrm{H}, \mathrm{H}_{4}, E\right), 3.19\left(\mathrm{dd}, J_{\mathrm{H} 5, \mathrm{H} 6}=7.2 \mathrm{~Hz}, J_{\mathrm{H} 5, \mathrm{H} 4}=\right.$ 
$\left.2.4 \mathrm{~Hz}, 1 \mathrm{H}, \mathrm{H}_{4}, Z\right), 2.84-2.79\left(\mathrm{~m}, 1 \mathrm{H}, \mathrm{H}_{3}, \mathrm{Z}\right), 2.67$ (ddd, $J_{\mathrm{H} 3, \mathrm{H} 3}{ }=14.4 \mathrm{~Hz}, J_{\mathrm{H} 3, \mathrm{H} 2}=6.6$ $\left.\mathrm{Hz}, J_{\mathrm{H} 3, \mathrm{H} 4}=3.0 \mathrm{~Hz}, 1 \mathrm{H}, \mathrm{H}_{3}, E\right), 2.51-2.46\left(\mathrm{~m}, 1 \mathrm{H}, \mathrm{H}_{3}, Z\right), 2.39$ (ddd, $J_{\mathrm{H} 3}, \mathrm{H} 3=14.4 \mathrm{~Hz}$, $\left.J_{\mathrm{H} 3^{\prime}, \mathrm{H} 4}=8.4 \mathrm{~Hz}, J_{\mathrm{H} 3^{\prime}, \mathrm{H} 2}=7.2 \mathrm{~Hz}, 1 \mathrm{H}, \mathrm{H}_{3^{\prime}}, E\right), 1.25\left(\mathrm{~d}, J_{\mathrm{H} 7, \mathrm{H} 6}=6.6 \mathrm{~Hz}, 3 \mathrm{H}, \mathrm{H}_{7}, E\right), 1.21(\mathrm{~d}$, $\left.J_{\mathrm{H} 7, \mathrm{H} 6}=6.6 \mathrm{~Hz}, 3 \mathrm{H}, \mathrm{H}_{7}, Z\right) .{ }^{13} \mathrm{C}\left\{{ }^{1} \mathrm{H}\right\} \mathrm{NMR}\left(151 \mathrm{MHz}, \mathrm{CD}_{3} \mathrm{OD}\right): \delta 139.2(\mathrm{~s}, \mathrm{Ph}), 139.0(\mathrm{~s}$, $\mathrm{Ph}), 133.3$ (s, $\left.\mathrm{C}_{1}, E\right), 131.5$ (s, $\left.\mathrm{C}_{1}, Z\right), 130.4$ (s, C $\left.2, Z\right), 129.9$ (s, Ph), 129.5 (s, Ph), 129.1 (s, $\left.\mathrm{C}_{2}, E\right), 128.2(\mathrm{~s}, \mathrm{Ph}), 127.9(\mathrm{~s}, \mathrm{Ph}), 127.6(\mathrm{~s}, \mathrm{Ph}), 127.0(\mathrm{~s}, \mathrm{Ph}), 78.1$ (s, $\mathrm{C}_{5}, E$ or $Z$ ), 77.9 (s, $\mathrm{C}_{5}, E$ or $Z$ ), 73.2 (s, $\mathrm{C}_{4}, E$ or $Z$ ), 72.8 (s, $\mathrm{C}_{4}, E$ or $Z$ ), 67.7 (s, $\left.\mathrm{C}_{6}, E \& Z\right), 38.4$ (s, $\left.\mathrm{C}_{3}, E\right), 34.0\left(\mathrm{~s}, \mathrm{C}_{3}, Z\right), 20.0\left(\mathrm{~s}, \mathrm{C}_{7}, E\right.$ or $\left.Z\right), 19.9$ (s, $\mathrm{C}_{7}, E$ or $Z$ ).

Relative stereochemistry determined by Kishi analysis of primary reduction product.

[i.e. No inversion $\left(\mathrm{O}_{4}\right.$ to $\left.\mathrm{C}_{7}\right)$ ]

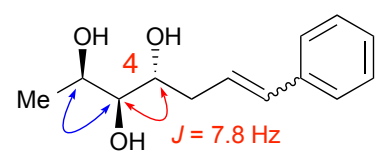

$J=2.4 \mathrm{~Hz}$

$C_{4}$ not inverted

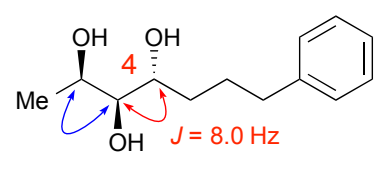

$J=3.0 \mathrm{~Hz}$

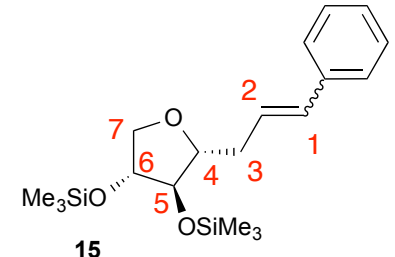

cyclization occurs from $\mathrm{O}_{4}$ to $\mathrm{C}_{7}$ with retention of stereochemistry

\section{Preparation of carbocycle product 13:}

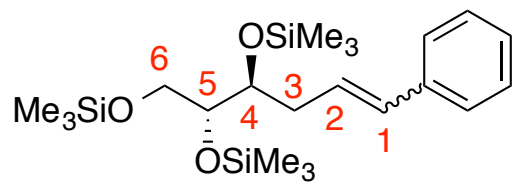

12 ribo- $(55: 45 E / Z)$
1) $10 \mathrm{~mol} \% \mathrm{~B}\left(\mathrm{C}_{6} \mathrm{~F}_{5}\right)_{3}$ 1.5 equiv. $\mathrm{Me}_{2} \mathrm{EtSiH}$, $\mathrm{CH}_{2} \mathrm{Cl}_{2},-30^{\circ} \mathrm{C}, 12 \mathrm{~h}$;

2) Dowex, $\mathrm{MeOH}$ $25^{\circ} \mathrm{C}, 2 \mathrm{~h}$

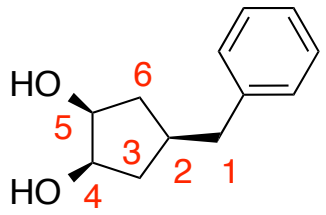

13

In a nitrogen-filled glovebox, a 1-dram vial was charged with $\mathrm{Me}_{3} \mathrm{Si}$-styryl 2-deoxyribose 12 (42.2 $\mathrm{mg}, 0.0993 \mathrm{mmol}, 1.00$ equiv.) and a stirbar. A separate 1-dram vial was charged with $\mathrm{B}\left(\mathrm{C}_{6} \mathrm{~F}_{5}\right)_{3}\left(4.9 \mathrm{mg}, 0.0096 \mathrm{mmol}, 0.096\right.$ equiv.) and $\mathrm{CH}_{2} \mathrm{Cl}_{2}(0.5 \mathrm{~mL})$. The vials were sealed with a septa cap and removed from the glovebox. The vials were allowed to cool to $-30{ }^{\circ} \mathrm{C}$ (dry ice/acetone bath). The $\mathrm{B}\left(\mathrm{C}_{6} \mathrm{~F}_{5}\right)_{3}$ solution was transferred to the substrate by syringe and allowed to stir as dimethylethylsilane $(20.0 \mu \mathrm{L}, 0.1519$ mmol, 1.5 equiv.) was added by microliter syringe. The vial was wrapped with electrical tape and transferred to a $-30{ }^{\circ} \mathrm{C}$ cryobath and allowed to stir. After 12 hours, $\mathrm{NEt}_{3}(0.05$ $\mathrm{mL}$ ) was added by syringe through the septa and the stir bar was removed, rinsing with $\mathrm{CH}_{2} \mathrm{Cl}_{2}(2 \mathrm{~mL})$. The quenched reaction mixture was concentrated under vacuum and then loaded onto an alumina plug (neutral, $10 \% \mathrm{H}_{2} \mathrm{O} ; 2 \times 1 \mathrm{~cm}$ ) with $100 \%$ hexanes $(1.5 \mathrm{~mL}$ ) then 95:5 hexanes:diethyl ether $(1.5 \mathrm{~mL})$, eluting with 95:5 hexanes:diethyl ether $(10 \mathrm{~mL})$ into a tared vial. The solution was concentrated under vacuum to afford a colorless oil 
(53.4 mg). The vial containing the silyl substrate was equipped with a stir bar and charged with Dowex ${ }^{\circledR}$ resin $(\sim 10$ beads $)$. Methanol $(2.0 \mathrm{~mL})$ was added by syringe and the resulting solution was allowed to stir at $25^{\circ} \mathrm{C}$. After $2 \mathrm{~h}$, the solution was passed through sand $(0.1 \mathrm{~cm})$ to remove the Dowex ${ }^{\circledR}$ beads into a tared vial, rinsing with $100 \%$ methanol. The product was purified by silica gel chromatography $(4 \times 1 \mathrm{~cm}$ column $)$ using gradient elution $\left(100 \% \mathrm{CH}_{2} \mathrm{Cl}_{2}\right.$ to $50: 1$ to $45: 1$ to $\left.40: 1 \mathrm{CH}_{2} \mathrm{Cl}_{2}: \mathrm{MeOH}\right)$ to afford carbocycle from $\mathrm{Me}_{3} \mathrm{Si}$-styryl 2-deoxyribose $\mathbf{1 3}$ as a colorless oil $(11.5 \mathrm{mg}, 60 \%$ overall yield over 2 steps, $0.0598 \mathrm{mmol}, 90: 10 \mathrm{dr}$ ). Note: stereochemistry is assigned by analogy to carbocycle $\mathbf{2 3}$ vide infra.

\section{Data for 13}

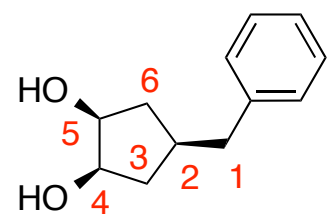

13

(1R,2S)-4-benzylcyclopentane-1,2-diol (13): ${ }^{1} \mathrm{H}$ NMR (600 MHz, $\left.\mathrm{CD}_{3} \mathrm{OD}\right): \delta 7.26$ (t, $J$ $=7.2 \mathrm{~Hz}, 2 \mathrm{H}, \mathrm{Ph}), 7.19-7.15(\mathrm{~m}, 3 \mathrm{H}, \mathrm{Ph}), 3.94-3.91\left(\mathrm{~m}, 2 \mathrm{H}, \mathrm{H}_{4} \& \mathrm{H}_{5}\right), 2.80\left(\mathrm{~d}, J_{\mathrm{H} 1, \mathrm{H} 2}=\right.$ $7.8 \mathrm{~Hz}, 2 \mathrm{H}, \mathrm{H}_{1}$ ), 2.14 (app quintet, $J_{\mathrm{H} 2, \mathrm{H} 3}=J_{\mathrm{H} 2, \mathrm{H} 6}=J_{\mathrm{H} 2, \mathrm{H} 1}=8.4 \mathrm{~Hz}, 1 \mathrm{H}, \mathrm{H}_{2}$ ), 1.96 (ddd, $J_{\mathrm{H} 3 / 6, \mathrm{H}^{3} / 6^{\prime}}=14.4 \mathrm{~Hz}, J_{\mathrm{H} 3 / 6, \mathrm{H} 2}=8.4 \mathrm{~Hz}, J_{\mathrm{H} 3 / 6, \mathrm{H} 4 / 5}=6.6 \mathrm{~Hz}, 2 \mathrm{H}, \mathrm{H}_{3} \& \mathrm{H}_{6}$ ), 1.44 (ddd, $\left.J_{\mathrm{H} 3^{\prime} / 6^{\prime}, \mathrm{H} 3 / 6}=14.4 \mathrm{~Hz}, J_{\mathrm{H}^{\prime} / 66^{\prime}, \mathrm{H} 2}=8.4 \mathrm{~Hz}, J_{\mathrm{H}^{\prime} / 6, \mathrm{H} 4 / 5}=J_{\mathrm{H}}=6.0 \mathrm{~Hz}, 2 \mathrm{H}, \mathrm{H}_{3^{\prime}}, \& \mathrm{H}_{6^{\prime}}\right) .{ }^{13} \mathrm{C}\left\{{ }^{1} \mathrm{H}\right\}$ NMR (151 MHz, CD ${ }_{3} \mathrm{OD}$ ): $\delta 142.9$ (s, Ar), 129.8 (s, Ar), 129.2 (s, Ar), 126.8 (s, Ar), $74.5\left(\mathrm{~s}, \mathrm{C}_{5} \& \mathrm{C}_{4}\right), 44.4\left(\mathrm{~s}, \mathrm{C}_{1}\right), 38.5\left(\mathrm{~s}, \mathrm{C}_{3} \& \mathrm{C}_{6}\right), 36.8\left(\mathrm{~s}, \mathrm{C}_{2}\right)$. HRMS $\left(\mathrm{ESI}^{+}\right)$: Calcd for $\mathrm{C}_{12} \mathrm{H}_{16} \mathrm{O}_{2} \mathrm{Na}[\mathrm{M}+\mathrm{Na}]^{+}: 215.1043$; Found: 215.1050 . Specific rotation: $[\alpha]^{\mathrm{D}} 24.9+14 . \quad(\mathrm{c}=$ $\left.0.24 \mathrm{CH}_{3} \mathrm{OH}\right)$.

\section{Preparation of carbocycle product 15:}

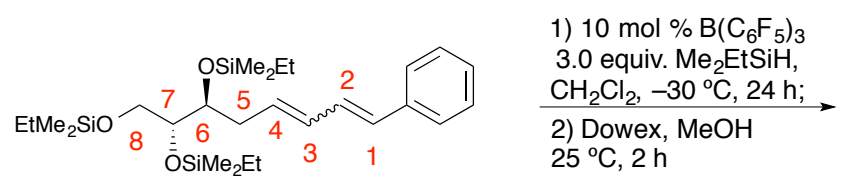

14 ribo- (76:24 E,E/E,Z)

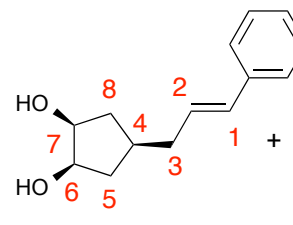

15

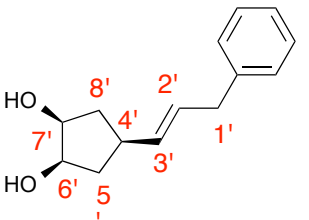

15

In a nitrogen-filled glovebox, a 1-dram vial was charged with $\mathrm{Me}_{2} \mathrm{EtSi}$-2-deoxyribose diene 14 (49.2 mg, $0.100 \mathrm{mmol}, 1.00$ equiv.) and a magnetic stir bar. A separate 1-dram vial was charged with $\mathrm{B}\left(\mathrm{C}_{6} \mathrm{~F}_{5}\right)_{3}\left(5.1 \mathrm{mg}, 0.010 \mathrm{mmol}, 0.10\right.$ equiv.) and $\mathrm{CH}_{2} \mathrm{Cl}_{2}(0.5 \mathrm{~mL})$. The vials were sealed with a septa cap and removed from the glovebox. The vials were allowed to cool to $-30{ }^{\circ} \mathrm{C}$ (dry ice/acetone bath). The $\mathrm{B}\left(\mathrm{C}_{6} \mathrm{~F}_{5}\right)_{3}$ solution was transferred to the substrate by syringe and allowed to stir as dimethylethylsilane $(40.0 \mu \mathrm{L}, 0.300 \mathrm{mmol}$, 
3.0 equiv.) was added by microliter syringe. The vial was wrapped with electrical tape and transferred to a $-30{ }^{\circ} \mathrm{C}$ cryobath and allowed to stir. After 12 hours, $\mathrm{NEt}_{3}(0.05 \mathrm{~mL})$ was added by syringe through the septa and the stir bar was removed, rinsing with $\mathrm{CH}_{2} \mathrm{Cl}_{2}(0.2 \mathrm{~mL})$. The quenched reaction mixture was concentrated under vacuum and then loaded onto an alumina plug (neutral, $10 \% \mathrm{H}_{2} \mathrm{O} ; 2 \times 1 \mathrm{~cm}$ ) with $100 \%$ hexanes $(1.5$ $\mathrm{mL})$ then $95: 5$ hexanes:diethyl ether $(1.5 \mathrm{~mL})$, eluting with $95: 5$ hexanes:diethyl ether $(10$ $\mathrm{mL}$ ) into a vial. The solution was concentrated under vacuum to afford a colorless oil $(55.7 \mathrm{mg})$. The vial containing the silyl substrate was equipped with a stir bar and charged with Dowex ${ }^{\circledR}$ resin $(\sim 10$ beads $)$. Methanol $(2.0 \mathrm{~mL})$ was added by syringe and the resulting solution was allowed to stir at $25^{\circ} \mathrm{C}$. After $2 \mathrm{~h}$, the solution was passed through sand $(0.1 \mathrm{~cm})$ to remove the Dowex ${ }^{\circledR}$ beads into a tared vial, rinsing with $100 \%$ methanol. The product was purified by silica gel chromatography $(4 \times 1 \mathrm{~cm}$ column) using gradient elution $\left(100 \% \mathrm{CH}_{2} \mathrm{Cl}_{2}\right.$ to $50: 1$ to $45: 1$ to $\left.40: 1 \mathrm{CH}_{2} \mathrm{Cl}_{2}: \mathrm{MeOH}\right)$ to afford carbocycle from $\mathrm{Me}_{2}$ EtSi-styryl-2-deoxyribose 15 as a white solid $(15.4 \mathrm{mg}, 71 \%$ overall yield over 2 steps, $0.0709 \mathrm{mmol},>98: 2 \mathrm{dr}$, and 55:45 $\mathrm{S}_{\mathrm{N}} 2{ }^{\prime} / \mathrm{S}_{\mathrm{N}} 2$ '"). Note: stereochemistry is assigned by analogy to carbocycle $\mathbf{2 3}$ vide infra.

\section{$\underline{\text { Data for } 15}$}

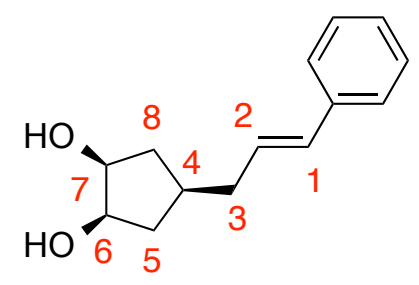

15

(1R,2S)-4-cinnamylcyclopentane-1,2-diol (15): ${ }^{1} \mathrm{H}$ NMR (600 MHz, $\left.\mathrm{CD}_{3} \mathrm{OD}\right): \delta 7.33$ (app d, $J=7.3 \mathrm{~Hz}, 1 \mathrm{H}, \mathrm{Ph}$ ), 7.28-7.22 (m, 2H, Ph), 7.16 (app t, $J=8.2 \mathrm{~Hz}, 2 \mathrm{H}, \mathrm{Ph}$ ), 6.40 $\left(\mathrm{d}, J_{\mathrm{H} 1, \mathrm{H} 2}=15.8 \mathrm{~Hz}, 1 \mathrm{H}, \mathrm{H}_{1}\right), 6.22\left(\mathrm{dt}, J_{\mathrm{H} 2, \mathrm{H} 1}=15.7 \mathrm{~Hz}, J_{\mathrm{H} 2, \mathrm{H} 3}=7.1 \mathrm{~Hz}, 1 \mathrm{H}, \mathrm{H}_{2}\right), 3.96-$ $3.88\left(\mathrm{~m}, 2 \mathrm{H}, \mathrm{H}_{6} \& \mathrm{H}_{7}\right), 2.31\left(\right.$ app t, $\left.J_{\mathrm{H} 3, \mathrm{H} 4}=J_{\mathrm{H} 3, \mathrm{H} 2}=7.1 \mathrm{~Hz}, 2 \mathrm{H}, \mathrm{H}_{3}\right), 2.09-2.02(\mathrm{~m}, 2 \mathrm{H}$, $\left.\mathrm{H}_{8} \& \mathrm{H}_{5}\right), 2.02-1.92\left(\mathrm{~m}, 1 \mathrm{H}, \mathrm{H}_{4}\right), 1.48-1.43\left(\mathrm{~m}, 2 \mathrm{H}, \mathrm{H}_{8}, \& \mathrm{H}_{5}\right) .{ }^{13} \mathrm{C}\left\{{ }^{1} \mathrm{H}\right\}$ NMR $(151$ $\left.\mathrm{MHz}, \mathrm{CD}_{3} \mathrm{OD}\right): \delta 137.8(\mathrm{~s}, \mathrm{Ph}), 130.6\left(\mathrm{~s}, \mathrm{C}_{1}\right), 129.1\left(\mathrm{~s}, \mathrm{C}_{2}\right), 128.1(\mathrm{~s}, \mathrm{Ph}), 126.5(\mathrm{~s}, \mathrm{Ph})$, $125.6(\mathrm{~s}, \mathrm{Ph}), 73.2\left(\mathrm{~s}, \mathrm{C}_{6} \& \mathrm{C}_{7}\right), 41.8\left(\mathrm{~s}, \mathrm{C}_{3}\right), 39.4\left(\mathrm{~s}, \mathrm{C}_{5} \& \mathrm{C}_{8}\right), 34.2\left(\mathrm{~s}, \mathrm{C}_{4}\right)$. HRMS $\left(\mathrm{ESI}^{+}\right)$: Calcd for $\mathrm{C}_{14} \mathrm{H}_{18} \mathrm{O}_{3} \mathrm{Na}[\mathrm{M}+\mathrm{Na}]^{+}: 241.1199$; Found: 241.1205. Specific rotation: $[\alpha]^{\mathrm{D}}{ }_{25.8}+0.50\left(\mathrm{c}=0.86 \mathrm{CH}_{3} \mathrm{OH}\right)$.

\section{Data for 15,}




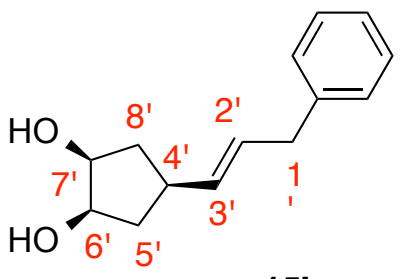

15'

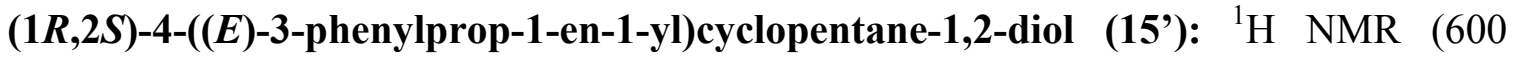
$\mathrm{MHz}, \mathrm{CD}_{3} \mathrm{OD}$ ): $\delta 7.33$ (app d, $\left.J=7.3 \mathrm{~Hz}, 1 \mathrm{H}, \mathrm{Ph}\right), 7.28-7.22$ (m, 2H, Ph), 7.16 (app t, $J$ $=8.2 \mathrm{~Hz}, 2 \mathrm{H}, \mathrm{Ph}), 5.53-5.51\left(\mathrm{~m}, 2 \mathrm{H}, \mathrm{H}_{2}, \& \mathrm{H}_{3}\right), 3.96-3.88\left(\mathrm{~m}, 2 \mathrm{H}, \mathrm{H}_{6}, \& \mathrm{H}_{7^{\prime}}\right), 3.29(\mathrm{~d}$, $\left.J_{\mathrm{H} 1^{\prime}, \mathrm{H} 2^{\prime}}=4.8 \mathrm{~Hz}, 2 \mathrm{H}, \mathrm{H}_{1}{ }^{\prime}\right), 2.48-2.38\left(\mathrm{~m}, 1 \mathrm{H}, \mathrm{H}_{4}{ }^{\prime}\right), 2.09-2.02\left(\mathrm{~m}, 2 \mathrm{H}, \mathrm{H}_{8}, \& \mathrm{H}_{5}{ }^{\prime}\right), 1.56-$ 1.49 (m, 2H, $\mathrm{H}_{5}$, \& $\mathrm{H}_{8}$, ). ${ }^{13} \mathrm{C}\left\{{ }^{1} \mathrm{H}\right\}$ NMR (151 MHz, $\left.\mathrm{CD}_{3} \mathrm{OD}\right): 142.2$ (s, Ph), 137.7 (s, $\mathrm{C}_{2}$, or $\mathrm{C}_{3}$ ), $129.4(\mathrm{~s}, \mathrm{Ph}), 128.7$ (s, Ph), 127.8 (s, $\mathrm{C}_{2}$, or $\left.\mathrm{C}_{3}{ }^{\prime}\right), 126.9(\mathrm{~s}, \mathrm{Ph}), 74.4\left(\mathrm{~s}, \mathrm{C}_{6}, \& \mathrm{C}_{7}{ }^{\prime}\right)$, $39.8\left(\mathrm{~s}, \mathrm{C}_{5}, \& \mathrm{C}_{8}\right), 38.3\left(\mathrm{~s}, \mathrm{C}_{1}\right), 38.1\left(\mathrm{~s}, \mathrm{C}_{4}\right)$ ).

\section{Preparation of carbocycle product 20 from 16:}
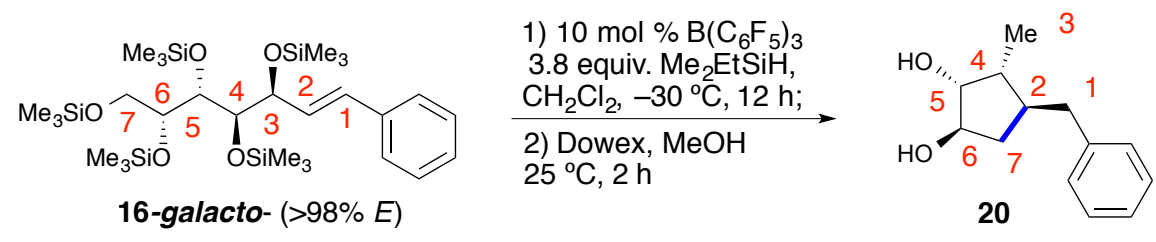

In a nitrogen-filled glovebox, a 1-dram vial was charged with $\mathrm{Me}_{3} \mathrm{Si}$-styrylgalactose 16 (61.4 mg, $0.0998 \mathrm{mmol}, 1.00$ equiv.) and a stirbar. A separate 1-dram vial was charged with $\mathrm{B}\left(\mathrm{C}_{6} \mathrm{~F}_{5}\right)_{3}\left(5.0 \mathrm{mg}, 0.0098 \mathrm{mmol}, 0.098\right.$ equiv.) and $\mathrm{CH}_{2} \mathrm{Cl}_{2}(0.5 \mathrm{~mL})$. The vials were sealed with a septa cap and removed from the glovebox. The vials were allowed to cool to $-30{ }^{\circ} \mathrm{C}$ (dry ice/acetone bath). The $\mathrm{B}\left(\mathrm{C}_{6} \mathrm{~F}_{5}\right)_{3}$ solution was transferred to the substrate by syringe and allowed to stir as dimethylethylsilane $(50.0 \mu \mathrm{L}, 0.379 \mathrm{mmol}, 3.79$ equiv.) was added by microliter syringe. The vial was wrapped with electrical tape and transferred to a $-30{ }^{\circ} \mathrm{C}$ cryobath and allowed to stir. After 12 hours, $\mathrm{NEt}_{3}(0.05 \mathrm{~mL})$ was added by syringe through the septa and the stir bar was removed, rinsing with $\mathrm{CH}_{2} \mathrm{Cl}_{2}(2$ $\mathrm{mL})$. The quenched reaction mixture was concentrated under vacuum and then loaded onto an alumina plug (neutral, $10 \% \mathrm{H}_{2} \mathrm{O} ; 2 \times 1 \mathrm{~cm}$ ) with $100 \%$ hexanes $(1.5 \mathrm{~mL})$ then 95:5 hexanes:diethyl ether $(1.5 \mathrm{~mL})$, eluting with 95:5 hexanes:diethyl ether $(10 \mathrm{~mL})$ into a tared vial. The solution was concentrated under vacuum to afford a colorless oil (71.2 $\mathrm{mg}$ ). The vial containing the silyl substrate was equipped with a stir bar and charged with Dowex $^{\circledR}$ resin $(\sim 10$ beads). Methanol $(2.0 \mathrm{~mL})$ was added by syringe and the resulting solution was allowed to stir at $25^{\circ} \mathrm{C}$. After $2 \mathrm{~h}$, the solution was passed through sand $(0.1$ $\mathrm{cm})$ to remove the Dowex ${ }^{\circledR}$ beads into a tared vial, rinsing with $100 \%$ methanol. The product was purified by silica gel chromatography (4 x $1 \mathrm{~cm}$ column) using gradient elution $\left(100 \% \mathrm{CH}_{2} \mathrm{Cl}_{2}\right.$ to $50: 1$ to $45: 1$ to $40: 1$ to $\left.35: 1 \mathrm{CH}_{2} \mathrm{Cl}_{2}: \mathrm{MeOH}\right)$ to afford 
carbocycle from $\mathrm{Me}_{3} \mathrm{Si}$-styrylgalactose 20 as a white solid (16.9 mg, 82\% overall yield over 2 steps, $0.0819 \mathrm{mmol})$.

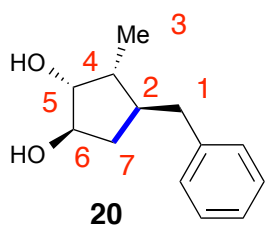

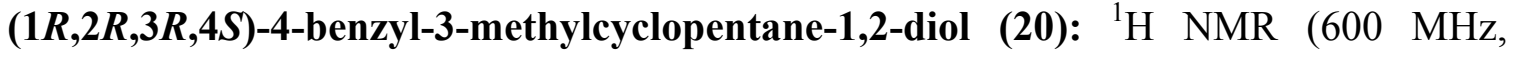
$\mathrm{CD}_{3} \mathrm{OD}$ ): $\delta 7.26$ (t, $\left.J=7.8 \mathrm{~Hz}, 2 \mathrm{H}, \mathrm{Ph}\right), 7.19$ (d, $\left.J=7.2 \mathrm{~Hz}, 2 \mathrm{H}, \mathrm{Ph}\right), 7.16$ (t, $J=7.2 \mathrm{~Hz}$, $1 \mathrm{H}, \mathrm{Ph}), 3.92\left(\mathrm{ddd}, J_{\mathrm{H} 6, \mathrm{H} 7}=6.6 \mathrm{~Hz}, J_{\mathrm{H} 6, \mathrm{H} 7}=4.2 \mathrm{~Hz}, J_{\mathrm{H} 6, \mathrm{H} 5}=2.4 \mathrm{~Hz}, 1 \mathrm{H}, \mathrm{H}_{6}\right), 3.81(\mathrm{dd}$, $\left.J_{\mathrm{H} 5, \mathrm{H} 4}=4.8 \mathrm{~Hz}, J_{\mathrm{H} 5, \mathrm{H} 6}=2.4 \mathrm{~Hz}, 1 \mathrm{H}, \mathrm{H}_{5}\right), 2.88\left(\mathrm{dd}, J_{\mathrm{H} 1, \mathrm{H} 1}=13.2 \mathrm{~Hz}, J_{\mathrm{H} 1, \mathrm{H} 2}=5.4 \mathrm{~Hz}, 1 \mathrm{H}\right.$,

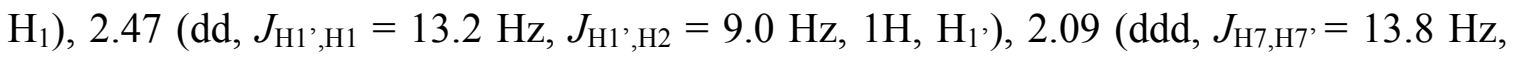
$\left.J_{\mathrm{H} 7, \mathrm{H} 2}=8.4 \mathrm{~Hz}, J_{\mathrm{H} 7, \mathrm{H} 6}=6.6 \mathrm{~Hz}, 1 \mathrm{H}, \mathrm{H}_{7}\right), 1.96-1.89\left(\mathrm{~m}, 1 \mathrm{H}, \mathrm{H}_{2}\right), 1.89-1.83\left(\mathrm{~m}, 1 \mathrm{H}, \mathrm{H}_{4}\right)$, $1.24\left(\mathrm{ddd}, J_{\mathrm{H} 7^{\prime}, \mathrm{H} 7}=13.2 \mathrm{~Hz}, J_{\mathrm{H}^{\prime}, \mathrm{H} 2}=8.4 \mathrm{~Hz}, J_{\mathrm{H} 7^{\prime}, \mathrm{H} 6}=4.2 \mathrm{~Hz}, 1 \mathrm{H}, \mathrm{H}_{7}\right), 0.98\left(\mathrm{~d}, J_{\mathrm{H} 3, \mathrm{H} 4}=\right.$ $\left.7.2 \mathrm{~Hz}, 3 \mathrm{H}, \mathrm{H}_{3}\right) .{ }^{13} \mathrm{C}\left\{{ }^{1} \mathrm{H}\right\} \mathrm{NMR}\left(151 \mathrm{MHz}, \mathrm{CD}_{3} \mathrm{OD}\right): \delta 142.9(\mathrm{~s}, \mathrm{Ph}), 129.9(\mathrm{~s}, \mathrm{Ph}), 129.2$ $(\mathrm{s}, \mathrm{Ph}), 126.8(\mathrm{~s}, \mathrm{Ph}), 82.4\left(\mathrm{~s}, \mathrm{C}_{5}\right), 78.8\left(\mathrm{~s}, \mathrm{C}_{6}\right), 45.6\left(\mathrm{~s}, \mathrm{C}_{4}\right), 43.3\left(\mathrm{~s}, \mathrm{C}_{2}\right), 42.7\left(\mathrm{~s}, \mathrm{C}_{1}\right), 39.6$ $\left(\mathrm{s}, \mathrm{C}_{7}\right), 13.1\left(\mathrm{~s}, \mathrm{C}_{3}\right)$. HRMS $\left(\mathrm{ESI}^{+}\right)$: Calcd for $\mathrm{C}_{13} \mathrm{H}_{18} \mathrm{O}_{2} \mathrm{Na}[\mathrm{M}+\mathrm{Na}]^{+}$: 229.1199; Found: 229.1200. Specific rotation: $[\alpha]^{\mathrm{D}}{ }_{24.9}+58$. $\left(\mathrm{c}=0.42 \mathrm{CH}_{3} \mathrm{OH}\right)$.

\section{Preparation of carbocycle product 21 from 17:}

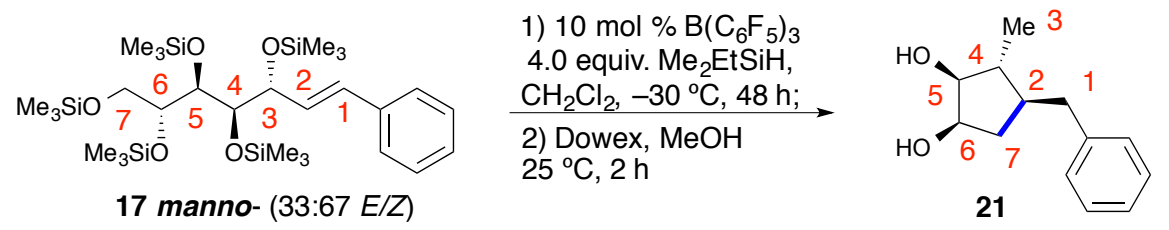

In a nitrogen-filled glovebox, a 1-dram vial was charged with $\mathrm{Me}_{3} \mathrm{Si}$-styrylmannose 17 (57.8 mg, $0.0940 \mathrm{mmol}, 1.00$ equiv.) and a magnetic stir bar. A separate 1-dram vial was charged with $\mathrm{B}\left(\mathrm{C}_{6} \mathrm{~F}_{5}\right)_{3}(5.0 \mathrm{mg}, 0.0098 \mathrm{mmol}, 0.10$ equiv. $)$ and $\mathrm{CH}_{2} \mathrm{Cl}_{2}(0.5 \mathrm{~mL})$. The vials were sealed with a septa cap and removed from the glovebox. The vials were allowed to cool to $-30{ }^{\circ} \mathrm{C}$ (dry ice/acetone bath). The $\mathrm{B}\left(\mathrm{C}_{6} \mathrm{~F}_{5}\right)_{3}$ solution was transferred to the substrate by syringe and allowed to stir as dimethylethylsilane $(50.0 \mu \mathrm{L}, 0.379 \mathrm{mmol}$, 4.03 equiv.) was added by microliter syringe. The vial was wrapped with electrical tape and transferred to a $-30{ }^{\circ} \mathrm{C}$ cryobath and allowed to stir. After 48 hours, $\mathrm{NEt}_{3}(0.05 \mathrm{~mL})$ was added by syringe through the septa and the stir bar was removed, rinsing with $\mathrm{CH}_{2} \mathrm{Cl}_{2}(2 \mathrm{~mL})$. The quenched reaction mixture was concentrated under vacuum and then loaded onto an alumina plug (neutral, $10 \% \mathrm{H}_{2} \mathrm{O} ; 2 \times 1 \mathrm{~cm}$ ) with $100 \%$ hexanes $(1.5 \mathrm{~mL})$ then 95:5 hexanes:diethyl ether $(1.5 \mathrm{~mL})$, eluting with 95:5 hexanes:diethyl ether $(10 \mathrm{~mL})$ into a tared vial. The solution was concentrated under vacuum to afford a colorless oil (64.1 mg, 85\% conv.). The vial containing the silyl substrate was equipped with a stir bar and charged with Dowex ${ }^{\circledR}$ resin $(\sim 10$ beads $)$. Methanol $(2.0 \mathrm{~mL})$ was added by syringe 
and the resulting solution was allowed to stir at $25^{\circ} \mathrm{C}$. After $2 \mathrm{~h}$, the solution was passed through sand $(0.1 \mathrm{~cm})$ to remove the Dowex ${ }^{\circledR}$ beads into a tared vial, rinsing with $100 \%$ methanol. The product was purified by silica gel chromatography (4 x $1 \mathrm{~cm}$ column) using gradient elution ( $100 \% \mathrm{CH}_{2} \mathrm{Cl}_{2}$ to $50: 1$ to $45: 1$ to $\left.40: 1 \mathrm{CH}_{2} \mathrm{Cl}_{2}: \mathrm{MeOH}\right)$ to afford carbocycle from $\mathrm{Me}_{3} \mathrm{Si}$-styrylmannose 21 as a white solid (14.3 $\mathrm{mg}, 74 \%$ overall yield over 2 steps, $0.0693 \mathrm{mmol}, 86: 14 \mathrm{dr}$ ). Note: stereochemistry is assigned by analogy to carbocycle 23 vide supra.

\section{Data for 21}

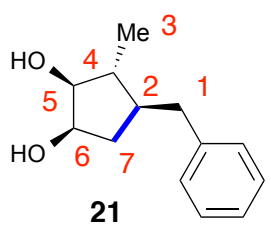

(1R,2S,3R,4S)-4-benzyl-3-methylcyclopentane-1,2-diol (21): ${ }^{1} \mathrm{H}$ NMR $(600 \mathrm{MHz}$, $\mathrm{CD}_{3} \mathrm{OD}$ ): $\delta 7.26$ (t, $\left.J=7.2 \mathrm{~Hz}, 2 \mathrm{H}, \mathrm{Ph}\right), 7.19$ (d, $\left.J=7.8 \mathrm{~Hz}, 2 \mathrm{H}, \mathrm{Ph}\right), 7.16$ (t, $J=7.2 \mathrm{~Hz}$, $1 \mathrm{H}, \mathrm{Ph}), 3.94\left(\mathrm{ddd}, J_{\mathrm{H} 6, \mathrm{H} 7}=10.2 \mathrm{~Hz}, J_{\mathrm{H} 6, \mathrm{H} 7}=4.8 \mathrm{~Hz}, J_{\mathrm{H} 6, \mathrm{H} 5}=4.8 \mathrm{~Hz}, 1 \mathrm{H}, \mathrm{H}_{6}\right), 3.40(\mathrm{dd}$, $\left.J_{\mathrm{H} 5, \mathrm{H} 4}=7.8 \mathrm{~Hz}, J_{\mathrm{H} 5, \mathrm{H} 6}=4.8 \mathrm{~Hz}, 1 \mathrm{H}, \mathrm{H}_{5}\right), 2.88\left(\mathrm{dd}, J_{\mathrm{H} 1, \mathrm{H} 1}=13.2 \mathrm{~Hz}, J_{\mathrm{H} 1, \mathrm{H} 2}=5.4 \mathrm{~Hz}, 1 \mathrm{H}\right.$,

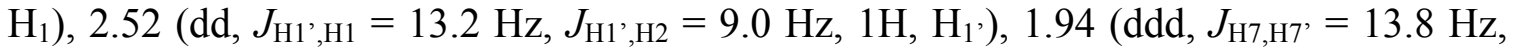
$\left.J_{\mathrm{H} 7, \mathrm{H} 6}=7.8 \mathrm{~Hz}, J_{\mathrm{H} 7, \mathrm{H} 2}=6.6 \mathrm{~Hz}, 1 \mathrm{H}, \mathrm{H}_{7}\right), 1.70-1.59\left(\mathrm{~m}, 2 \mathrm{H}, \mathrm{H}_{4} \& \mathrm{H}_{2}\right), 1.39\left(\mathrm{ddd}, J_{\mathrm{H} 7}{ }^{\prime}, \mathrm{H} 7\right.$ $\left.=12.0 \mathrm{~Hz}, J_{\mathrm{H} 7^{\prime}, \mathrm{H} 2}=7.8 \mathrm{~Hz}, J_{\mathrm{H} 7^{\prime}, \mathrm{H} 6}=4.2 \mathrm{~Hz}, 1 \mathrm{H}, \mathrm{H}_{7}{ }^{\prime}\right), 0.99\left(\mathrm{~d}, J_{\mathrm{H} 3, \mathrm{H} 4}=6.6 \mathrm{~Hz}, 3 \mathrm{H}, \mathrm{H}_{3}\right)$. ${ }^{13} \mathrm{C}\left\{{ }^{1} \mathrm{H}\right\}$ NMR (151 MHz, CD $\left.{ }_{3} \mathrm{OD}\right): \delta 142.6(\mathrm{~s}, \mathrm{Ph}), 129.9(\mathrm{~s}, \mathrm{Ph}), 129.2(\mathrm{~s}, \mathrm{Ph}), 126.8(\mathrm{~s}$, $\mathrm{Ph}), 81.5\left(\mathrm{~s}, \mathrm{C}_{5}\right), 72.9\left(\mathrm{~s}, \mathrm{C}_{6}\right), 45.0\left(\mathrm{~s}, \mathrm{C}_{4}\right), 44.6\left(\mathrm{~s}, \mathrm{C}_{2}\right), 38.1\left(\mathrm{~s}, \mathrm{C}_{7}\right), 17.3\left(\mathrm{~s}, \mathrm{C}_{3}\right)$. HRMS $\left(\mathrm{ESI}^{+}\right.$): Calcd for $\mathrm{C}_{13} \mathrm{H}_{18} \mathrm{O}_{2} \mathrm{Na}[\mathrm{M}+\mathrm{Na}]^{+}:$229.1199; Found: 229.1194. Specific rotation: $[\alpha]_{24.9}^{\mathrm{D}}+35$. $\left(\mathrm{c}=0.22 \mathrm{CH}_{3} \mathrm{OH}\right)$.

\section{Preparation of carbocycle product 21 from 18:}

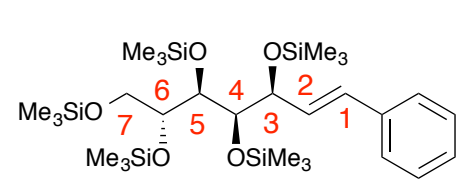

18 gluco- $(67: 33 E / Z)$

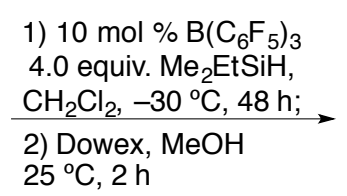

$25^{\circ} \mathrm{C}, 2 \mathrm{~h}$

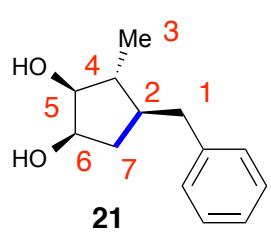

21

In a nitrogen-filled glovebox, a 1-dram vial was charged with $\mathrm{Me}_{3} \mathrm{Si}$-styrylmannose $\mathbf{1 8}$ (57.8 mg, $0.0940 \mathrm{mmol}, 1.00$ equiv.) and a magnetic stir bar. A separate 1-dram vial was charged with $\mathrm{B}\left(\mathrm{C}_{6} \mathrm{~F}_{5}\right)_{3}(5.0 \mathrm{mg}, 0.0098 \mathrm{mmol}, 0.10$ equiv. $)$ and $\mathrm{CH}_{2} \mathrm{Cl}_{2}(0.5 \mathrm{~mL})$. The vials were sealed with a septa cap and removed from the glovebox. The vials were allowed to cool to $-30{ }^{\circ} \mathrm{C}$ (dry ice/acetone bath). The $\mathrm{B}\left(\mathrm{C}_{6} \mathrm{~F}_{5}\right)_{3}$ solution was transferred to the substrate by syringe and allowed to stir as dimethylethylsilane $(50.0 \mu \mathrm{L}, 0.379 \mathrm{mmol}$, 4.03 equiv.) was added by microliter syringe. The vial was wrapped with electrical tape and transferred to a $-30{ }^{\circ} \mathrm{C}$ cryobath and allowed to stir. After 48 hours, $\mathrm{NEt}_{3}(0.05 \mathrm{~mL})$ 
was added by syringe through the septa and the stir bar was removed, rinsing with $\mathrm{CH}_{2} \mathrm{Cl}_{2}(2 \mathrm{~mL})$. The quenched reaction mixture was concentrated under vacuum and then loaded onto an alumina plug (neutral, $10 \% \mathrm{H}_{2} \mathrm{O} ; 2 \times 1 \mathrm{~cm}$ ) with $100 \%$ hexanes $(1.5 \mathrm{~mL})$ then 95:5 hexanes:diethyl ether $(1.5 \mathrm{~mL})$, eluting with 95:5 hexanes:diethyl ether $(10 \mathrm{~mL})$ into a tared vial. The solution was concentrated under vacuum to afford a colorless oil (64.1 mg, 85\% conv.). The vial containing the silyl substrate was equipped with a stir bar and charged with Dowex ${ }^{\circledR}$ resin $(\sim 10$ beads $)$. Methanol $(2.0 \mathrm{~mL})$ was added by syringe and the resulting solution was allowed to stir at $25^{\circ} \mathrm{C}$. After $2 \mathrm{~h}$, the solution was passed through sand $(0.1 \mathrm{~cm})$ to remove the Dowex ${ }^{\circledR}$ beads into a tared vial, rinsing with $100 \%$ methanol. The product was purified by silica gel chromatography (4 x $1 \mathrm{~cm}$ column) using gradient elution ( $100 \% \mathrm{CH}_{2} \mathrm{Cl}_{2}$ to $50: 1$ to $45: 1$ to $\left.40: 1 \mathrm{CH}_{2} \mathrm{Cl}_{2}: \mathrm{MeOH}\right)$ to afford carbocycle from $\mathrm{Me}_{3} \mathrm{Si}$-styrylmannose 21 as a white solid (14.3 mg, 74\% overall yield over 2 steps, $0.0693 \mathrm{mmol}, 86: 14 \mathrm{dr}$ ). Note: stereochemistry is assigned by analogy to carbocycle 23 vide supra.

\section{Data for 21}

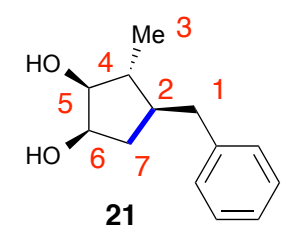

(1R,2S,3R,4S)-4-benzyl-3-methylcyclopentane-1,2-diol (21): ${ }^{1} \mathrm{H}$ NMR $(600 \mathrm{MHz}$, $\mathrm{CD}_{3} \mathrm{OD}$ ): $\delta 7.26$ (t, $\left.J=7.2 \mathrm{~Hz}, 2 \mathrm{H}, \mathrm{Ph}\right), 7.19$ (d, $\left.J=7.8 \mathrm{~Hz}, 2 \mathrm{H}, \mathrm{Ph}\right), 7.16$ (t, $J=7.2 \mathrm{~Hz}$, $1 \mathrm{H}, \mathrm{Ph}), 3.94\left(\mathrm{ddd}, J_{\mathrm{H} 6, \mathrm{H} 7}=10.2 \mathrm{~Hz}, J_{\mathrm{H} 6, \mathrm{H} 7}=4.8 \mathrm{~Hz}, J_{\mathrm{H} 6, \mathrm{H} 5}=4.8 \mathrm{~Hz}, 1 \mathrm{H}, \mathrm{H}_{6}\right), 3.40(\mathrm{dd}$, $\left.J_{\mathrm{H} 5, \mathrm{H} 4}=7.8 \mathrm{~Hz}, J_{\mathrm{H} 5, \mathrm{H} 6}=4.8 \mathrm{~Hz}, 1 \mathrm{H}, \mathrm{H}_{5}\right), 2.88\left(\mathrm{dd}, J_{\mathrm{H} 1, \mathrm{H} 1}=13.2 \mathrm{~Hz}, J_{\mathrm{H} 1, \mathrm{H} 2}=5.4 \mathrm{~Hz}, 1 \mathrm{H}\right.$,

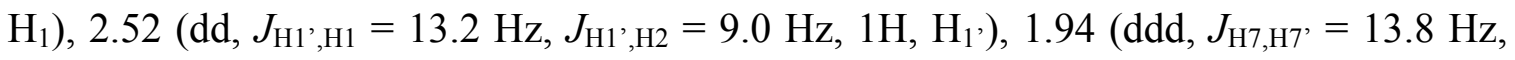
$\left.J_{\mathrm{H} 7, \mathrm{H} 6}=7.8 \mathrm{~Hz}, J_{\mathrm{H} 7, \mathrm{H} 2}=6.6 \mathrm{~Hz}, 1 \mathrm{H}, \mathrm{H}_{7}\right), 1.70-1.59\left(\mathrm{~m}, 2 \mathrm{H}, \mathrm{H}_{4} \& \mathrm{H}_{2}\right), 1.39\left(\mathrm{ddd}, J_{\mathrm{H} 7}{ }^{\prime}, \mathrm{H} 7\right.$ $\left.=12.0 \mathrm{~Hz}, J_{\mathrm{H} 7^{\prime}, \mathrm{H} 2}=7.8 \mathrm{~Hz}, J_{\mathrm{H}^{\prime}, \mathrm{H} 6}=4.2 \mathrm{~Hz}, 1 \mathrm{H}, \mathrm{H}_{7^{\prime}}\right), 0.99\left(\mathrm{~d}, J_{\mathrm{H} 3, \mathrm{H} 4}=6.6 \mathrm{~Hz}, 3 \mathrm{H}, \mathrm{H}_{3}\right)$. ${ }^{13} \mathrm{C}\left\{{ }^{1} \mathrm{H}\right\}$ NMR (151 MHz, CD $\left.\mathrm{CDD}_{3} \mathrm{O}\right) \delta 142.6(\mathrm{~s}, \mathrm{Ph}), 129.9(\mathrm{~s}, \mathrm{Ph}), 129.2(\mathrm{~s}, \mathrm{Ph}), 126.8(\mathrm{~s}$, $\mathrm{Ph}), 81.5\left(\mathrm{~s}, \mathrm{C}_{5}\right), 72.9\left(\mathrm{~s}, \mathrm{C}_{6}\right), 45.0\left(\mathrm{~s}, \mathrm{C}_{4}\right), 44.6\left(\mathrm{~s}, \mathrm{C}_{2}\right), 38.1\left(\mathrm{~s}, \mathrm{C}_{7}\right), 17.3\left(\mathrm{~s}, \mathrm{C}_{3}\right)$. HRMS $\left(\mathrm{ESI}^{+}\right.$): Calcd for $\mathrm{C}_{13} \mathrm{H}_{18} \mathrm{O}_{2} \mathrm{Na}[\mathrm{M}+\mathrm{Na}]^{+}:$229.1199; Found: 229.1194. Specific rotation: $[\alpha]_{24.9}^{\mathrm{D}}+35$. $\left(\mathrm{c}=0.22 \mathrm{CH}_{3} \mathrm{OH}\right)$.

\section{Preparation of carbocycle product 22 from 19:}

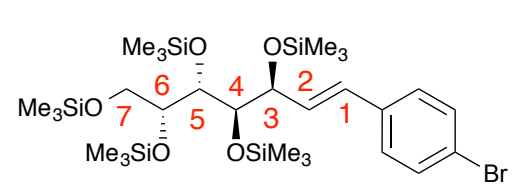

19 galacto- $(>98 \% E)$

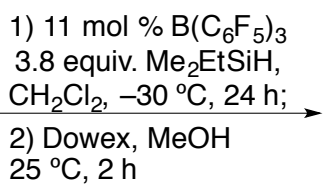

$25^{\circ} \mathrm{C}, 2 \mathrm{~h}$

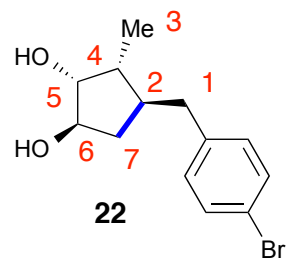


In a nitrogen-filled glovebox, a 1-dram vial was charged with $\mathrm{Me}_{3} \mathrm{Si}-p \mathrm{Br}$-styrylgalactose 19 (69.3 mg, $0.0998 \mathrm{mmol}, 1.00$ equiv.) and a stir bar. A separate 1-dram vial was charged with $\mathrm{B}\left(\mathrm{C}_{6} \mathrm{~F}_{5}\right)_{3}(5.5 \mathrm{mg}, 0.011 \mathrm{mmol}, 0.11$ equiv. $)$ and $\mathrm{CH}_{2} \mathrm{Cl}_{2}(0.5 \mathrm{~mL})$. The vials were sealed with a septa cap and removed from the glovebox. The vials were allowed to cool to $-30{ }^{\circ} \mathrm{C}$ (dry ice/acetone bath). The $\mathrm{B}\left(\mathrm{C}_{6} \mathrm{~F}_{5}\right)_{3}$ solution was transferred to the substrate by syringe and allowed to stir as dimethylethylsilane $(50.0 \mu \mathrm{L}, 0.379 \mathrm{mmol}$, 3.79 equiv.) was added by microliter syringe. The vial was wrapped with electrical tape and transferred to a $-30{ }^{\circ} \mathrm{C}$ cryobath and allowed to stir. After 24 hours, $\mathrm{NEt}_{3}(0.05 \mathrm{~mL})$ was added by syringe through the septa and the stir bar was removed, rinsing with $\mathrm{CH}_{2} \mathrm{Cl}_{2}(2 \mathrm{~mL})$. The quenched reaction mixture was concentrated under vacuum and then loaded onto an alumina plug (neutral, $10 \% \mathrm{H}_{2} \mathrm{O} ; 2 \times 1 \mathrm{~cm}$ ) with $100 \%$ hexanes $(1.5 \mathrm{~mL})$ then 95:5 hexanes:diethyl ether $(1.5 \mathrm{~mL})$, eluting with 95:5 hexanes:diethyl ether $(10 \mathrm{~mL})$ into a tared vial. The solution was concentrated under vacuum to afford a colorless oil (66.7 mg). The vial containing the silyl substrate was equipped with a stir bar and charged with Dowex ${ }^{\circledR}$ resin $(\sim 10$ beads). Methanol $(2.0 \mathrm{~mL})$ was added by syringe and the resulting solution was allowed to stir at $25{ }^{\circ} \mathrm{C}$. After $2 \mathrm{~h}$, the solution was passed through sand $(0.1 \mathrm{~cm})$ to remove the Dowex ${ }^{\circledR}$ beads into a tared vial, rinsing with $100 \%$ methanol. The product was purified by silica gel chromatography (4 x $1 \mathrm{~cm}$ column) using gradient elution ( $100 \% \mathrm{CH}_{2} \mathrm{Cl}_{2}$ to $50: 1$ to $45: 1$ to $\left.40: 1 \mathrm{CH}_{2} \mathrm{Cl}_{2}: \mathrm{MeOH}\right)$ to afford carbocycle from $\mathrm{Me}_{3} \mathrm{Si}$-pBr-styrylgalactose 22 as a white solid (20.3 mg, 71\% overall yield over 2 steps, $0.0712 \mathrm{mmol}$ ).

\section{Data for 22}

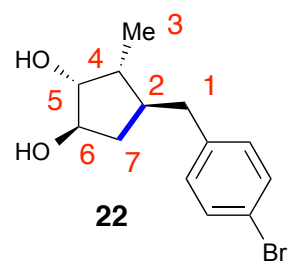

(1R,2R,3R,4S)-4-(4-bromobenzyl)-3-methylcyclopentane-1,2-diol (22): ${ }^{1} \mathrm{H}$ NMR (600 $\mathrm{MHz}, \mathrm{CD}_{3} \mathrm{OD}$ ): $\delta 7.41$ (d, $\left.J=7.8 \mathrm{~Hz}, 2 \mathrm{H}, \mathrm{Ar}\right), 7.12$ (d, $J=7.8 \mathrm{~Hz}, 2 \mathrm{H}, \mathrm{Ar}$ ), 3.92 (br s, $\left.1 \mathrm{H}, \mathrm{H}_{6}\right), 3.81\left(\mathrm{br} \mathrm{d}, J_{\mathrm{H} 5, \mathrm{H} 6}=3.6 \mathrm{~Hz}, 1 \mathrm{H}, \mathrm{H}_{5}\right), 2.85\left(\mathrm{dd}, J_{\mathrm{H} 1, \mathrm{H} 1}=13.2 \mathrm{~Hz}, J_{\mathrm{H} 1, \mathrm{H} 2}=4.8 \mathrm{~Hz}\right.$,

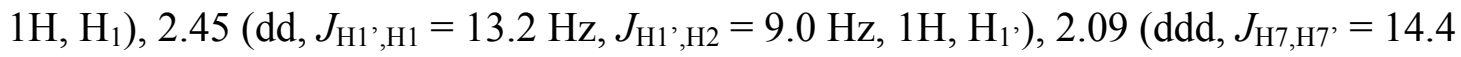
$\left.\mathrm{Hz}, J_{\mathrm{H} 7, \mathrm{H} 6}=7.8 \mathrm{~Hz}, J_{\mathrm{H} 7, \mathrm{H} 2}=6.6 \mathrm{~Hz}, 1 \mathrm{H}, \mathrm{H}_{7}\right), 1.94-1.89\left(\mathrm{~m}, 1 \mathrm{H}, \mathrm{H}_{2}\right), 1.88-1.82(\mathrm{~m}, 1 \mathrm{H}$, $\left.\mathrm{H}_{4}\right), 1.21\left(\mathrm{ddd}, J_{\mathrm{H} 7^{\prime}, \mathrm{H} 7}=13.2 \mathrm{~Hz}, J_{\mathrm{H} 7^{\prime}, \mathrm{H} 2}=7.8 \mathrm{~Hz}, J_{\mathrm{H} 7^{\prime}, \mathrm{H} 6}=4.2 \mathrm{~Hz}, 1 \mathrm{H}, \mathrm{H}_{7}{ }^{\prime}\right), 0.98(\mathrm{~d}$, $\left.J_{\mathrm{H} 3, \mathrm{H} 4}=7.2 \mathrm{~Hz}, 3 \mathrm{H}, \mathrm{H}_{3}\right) .{ }^{13} \mathrm{C}\left\{{ }^{1} \mathrm{H}\right\} \mathrm{NMR}\left(151 \mathrm{MHz}, \mathrm{CD}_{3} \mathrm{OD}\right): \delta 142.2(\mathrm{~s}, \mathrm{Ar}), 132.3(\mathrm{~s}$, Ar), 131.9 (s, Ar), 120.4 (s, Ar), 82.3 (s, C 5 ), 78.7 (s, $\left.\mathrm{C}_{6}\right), 45.4\left(\mathrm{~s}, \mathrm{C}_{2}\right), 43.2\left(\mathrm{~s}, \mathrm{C}_{4}\right), 41.9$ $\left(\mathrm{s}, \mathrm{C}_{1}\right), 39.5\left(\mathrm{~s}, \mathrm{C}_{7}\right), 13.1\left(\mathrm{~s}, \mathrm{C}_{3}\right)$. HRMS $\left(\mathrm{ESI}^{+}\right)$: Calcd for $\mathrm{C}_{13} \mathrm{H}_{17} \mathrm{O}_{2} \mathrm{BrNa}[\mathrm{M}+\mathrm{Na}]^{+}$: 307.0304; Found: 307.0310. Specific rotation: $[\alpha]^{\mathrm{D}}{ }_{24.1}+43.8\left(\mathrm{c}=0.535 \mathrm{CH}_{3} \mathrm{OH}\right)$. 
Preparation of carbocycle derivative 23 from 22:
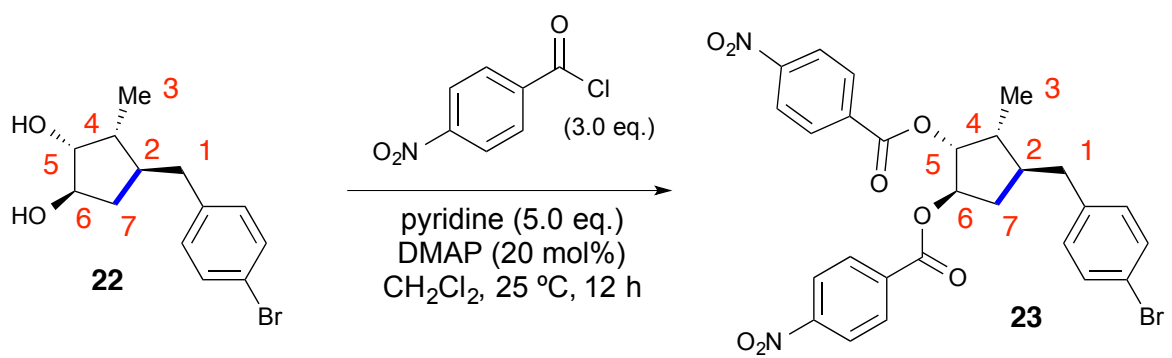

Under positive nitrogen flow, a septum capped 20-mL scintillation vial was charged with 22 (0.220 g, 1.00 mmol), 4-nitrobenzyl chloride (0.560 g, 3.00 mmol), 4dimethylaminopyridine (DMAP) (0.024 g, $0.20 \mathrm{mmol}$ ), and a magnetic stir bar. Dichloromethane was added by syringe $(10.0 \mathrm{~mL})$ followed by pyridine $(0.400 \mathrm{~mL}, 5.00$ mmol) by syringe. The septum was replaced with the vial cap and allowed to stir at $25^{\circ} \mathrm{C}$ for $12 \mathrm{~h}$. After stirring for $12 \mathrm{~h}, 10 \mathrm{~mL}$ of saturated sodium bicarbonate solution was added by syringe. The contents of the vial were then transferred to a separatory funnel rinsing with $\mathrm{Et}_{2} \mathrm{O}(3 \times 5 \mathrm{~mL})$. The aqueous layer was extracted with $\mathrm{Et}_{2} \mathrm{O}(3 \times 10 \mathrm{~mL})$ and combined. The combined organic extractions were washed with water $(3 \times 10 \mathrm{~mL})$, brine $(3 \times 10 \mathrm{~mL})$, and dried over sodium sulfate. The organic layer was filtered into a round bottom flask and solvent removed under vacuum to afford a white solid. Products were purified by silica gel chromatography ( $20 \times 3 \mathrm{~cm}$ column) using gradient elution (100\% hexanes to $30: 1$ to $20: 1$ to $15: 1$ to $10: 1$ to $7: 1$ to $5: 1$ hexanes:ethyl acetate) to afford $\quad(1 R, 2 R, 3 R, 4 S)$-4-(4-bromobenzyl)-3-methylcyclopentane-1,2-diyl bis(4nitrobenzoate) 23 as a white solid $(0.50 \mathrm{~g}, 86 \%$ overall yield over $0.86 \mathrm{mmol})$. This material was used to obtain a single crystal for X-ray analysis by vapor diffusion of hexanes into chloroform solution of $\mathbf{2 3}$.

\section{Data for 23}

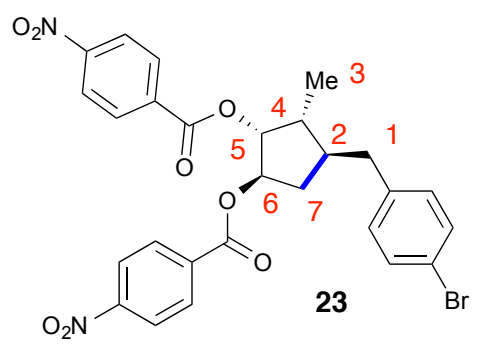

$(1 R, 2 R, 3 R, 4 S)$-4-(4-bromobenzyl)-3-methylcyclopentane-1,2-diyl

bis(4nitrobenzoate) (23): ${ }^{1} \mathrm{H}$ NMR $\left(600 \mathrm{MHz}, \mathrm{CDCl}_{3}\right): \delta 8.32-8.27(\mathrm{~m}, 4 \mathrm{H}), 8.24-8.18(\mathrm{~m}$, $2 \mathrm{H}), 8.16-8.10(\mathrm{~m}, 4 \mathrm{H}), 7.44-7.38(\mathrm{~m}, 2 \mathrm{H}), 7.10-7.03(\mathrm{~m}, 2 \mathrm{H}), 5.59$ (dd, $J=6.2,2.8 \mathrm{~Hz}$, $1 \mathrm{H}), 5.38$ (ddd, $J=7.2,6.0,2.9 \mathrm{~Hz}, 1 \mathrm{H}), 2.95$ (dd, $J=13.6,4.8 \mathrm{~Hz}, 1 \mathrm{H}), 2.55$ (dd, $J=$ 13.6, 8.7 Hz, 1H), $2.49(\mathrm{dd}, J=14.2,7.2 \mathrm{~Hz}, 1 \mathrm{H}), 2.29-2.12(\mathrm{~m}, 2 \mathrm{H}), 1.59-1.52(\mathrm{~m}, 1 \mathrm{H})$, $1.08(\mathrm{~d}, J=6.6 \mathrm{~Hz}, 3 \mathrm{H}) .{ }^{13} \mathrm{C}\left\{{ }^{1} \mathrm{H}\right\} \mathrm{NMR}\left(151 \mathrm{MHz}, \mathrm{CDCl}_{3}\right): \delta 163.9,163.8,150.6,150.6$, 
138.6, 135.0, 131.5, 130.8, 130.7, 130.7, 123.6, 123.6, 120.1, 81.7, 79.0, 44.7, 41.1, 39.4, $36.2,12.7$.

\section{Preparation of vinyl shift product 24 from 16:}

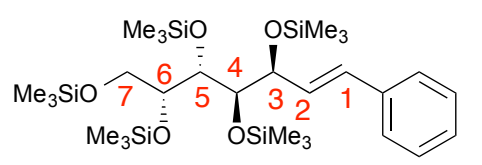

16 galacto- $(>98 E)$

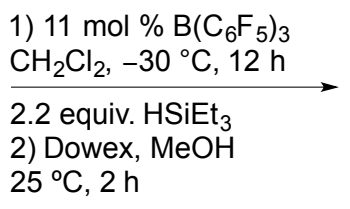

$25^{\circ} \mathrm{C}, 2 \mathrm{~h}$

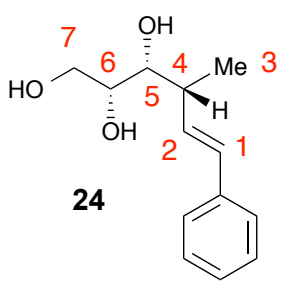

In a nitrogen-filled glovebox, a 1-dram vial was charged with $\mathrm{B}\left(\mathrm{C}_{6} \mathrm{~F}_{5}\right)_{3}(6.5 \mathrm{mg}, 0.013$ mmol, $11 \mathrm{~mol} \%)$ then triethylsilane $(40.0 \mu \mathrm{L}, 0.250 \mathrm{mmol}, 2.22$ equiv.) and then dichloromethane $(0.5 \mathrm{~mL})$. A separate 1 -dram vial was charged with $\mathrm{Me}_{3} \mathrm{Si}$ styrylgalactose 16 (69.6 mg, $0.113 \mathrm{mmol}, 1.00$ equiv.) and a magnetic stir bar. The vials were sealed with a septum cap and removed from the glovebox. Both vials were allowed to cool to $-30{ }^{\circ} \mathrm{C}$ (dry ice/acetone bath) and the silane solution was transferred to the substrate vial by syringe under $\mathrm{N}_{2}$. The vial cap was wrapped in electrical tape and the vial was transferred to a $-30{ }^{\circ} \mathrm{C}$ cryobath and allowed to stir. After $12 \mathrm{~h}, \mathrm{NEt}_{3}(0.05 \mathrm{~mL})$ was added by syringe through the septa and the solution was allowed to slowly warm to $25{ }^{\circ} \mathrm{C}$. The stir bar was removed, rinsing with $\mathrm{CH}_{2} \mathrm{Cl}_{2}(0.2 \mathrm{~mL})$ and the quenched reaction mixture was concentrated under vacuum and then loaded onto an alumina plug (neutral, $10 \% \mathrm{H}_{2} \mathrm{O} ; 2 \times 1 \mathrm{~cm}$ ) with $100 \%$ hexanes $(1.5 \mathrm{~mL})$ then $95: 5$ hexanes:diethyl ether $(1.5 \mathrm{~mL})$, eluting with 95:5 hexanes:diethyl ether $(10 \mathrm{~mL})$ into a tared vial. The solution was concentrated under vacuum to afford a colorless oil $(79.7 \mathrm{mg})$. Deprotection to alcohol was performed subsequently. The vial containing the silyl substrate was equipped with a stir bar and charged with Dowex ${ }^{\circledR}$ resin (10 beads). Methanol $(2.0 \mathrm{~mL})$ was added by syringe and the resulting solution was allowed to stir at $25^{\circ} \mathrm{C}$. After $2 \mathrm{~h}$, the solution was passed through sand $(0.1 \mathrm{~cm})$ to remove the Dowex ${ }^{\circledR}$ beads into a vial, rinsing with $100 \%$ methanol. The solution was concentrated to afford a white solid. Products were purified by silica gel chromatography ( $4 \times 1 \mathrm{~cm}$ column) using gradient elution (100\% $\mathrm{CH}_{2} \mathrm{Cl}_{2}$ to $50: 1$ to $45: 1$ to $40: 1$ to $35: 1 \mathrm{CH}_{2} \mathrm{Cl}_{2}: \mathrm{MeOH}$ ) to afford $(2 R, 3 R, 4 R, E)$-4-methyl-6-phenylhex-5-ene-1,2,3-triol 24 as a white solid (18.3 $\mathrm{mg}, 73 \%$ overall yield over 2 steps, $0.0825 \mathrm{mmol}$ ).

\section{Data for 24}

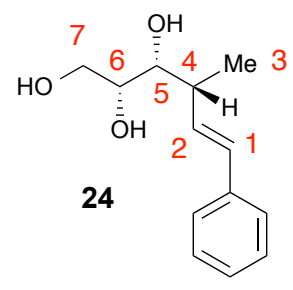


(2R,3R,4R,E)-4-methyl-6-phenylhex-5-ene-1,2,3-triol (24): ${ }^{1} \mathrm{H} \quad \mathrm{NMR}(600 \mathrm{MHz}$, $\mathrm{CD}_{3} \mathrm{OD}$ ): $\delta 7.39$ (d, $\left.J=7.8 \mathrm{~Hz}, 2 \mathrm{H}, \mathrm{Ph}\right), 7.29$ (t, $\left.J=7.8 \mathrm{~Hz}, 2 \mathrm{H}, \mathrm{Ph}\right), 7.18$ (t, $J=7.2 \mathrm{~Hz}$, $1 \mathrm{H}, \mathrm{Ph}), 6.46\left(\mathrm{~d}, J_{\mathrm{H} 1, \mathrm{H} 2}=16.2 \mathrm{~Hz}, 1 \mathrm{H}, \mathrm{H}_{1}\right), 6.34\left(\mathrm{dd}, J_{\mathrm{H} 2, \mathrm{H} 1}=16.2 \mathrm{~Hz}, J_{\mathrm{H} 2, \mathrm{H} 4}=8.4 \mathrm{~Hz}\right.$, $\left.1 \mathrm{H}, \mathrm{H}_{2}\right), 3.70-3.66\left(\mathrm{~m}, 2 \mathrm{H}, \mathrm{H}_{6} \& \mathrm{H}_{7}\right), 3.63-3.60\left(\mathrm{~m}, 1 \mathrm{H}, \mathrm{H}_{7}\right), 3.49-3.47\left(\mathrm{~m}, 1 \mathrm{H}, \mathrm{H}_{5}\right)$, 2.64-2.58 (m, 1H, $\left.\mathrm{H}_{4}\right), 1.17\left(\mathrm{~d}, J_{\mathrm{H} 3, \mathrm{H} 4}=7.2 \mathrm{~Hz}, 3 \mathrm{H}, \mathrm{H}_{3}\right) .{ }^{13} \mathrm{C}\left\{{ }^{1} \mathrm{H}\right\}$ NMR $(151 \mathrm{MHz}$, $\left.\mathrm{CD}_{3} \mathrm{OD}\right): \delta 139.2(\mathrm{~s}, \mathrm{Ph}), 133.8\left(\mathrm{~s}, \mathrm{C}_{2}\right), 131.4\left(\mathrm{~s}, \mathrm{C}_{1}\right), 129.5(\mathrm{~s}, \mathrm{Ph}), 127.9(\mathrm{~s}, \mathrm{Ph}), 127.1$

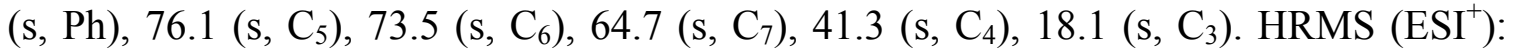
Calcd for $\mathrm{C}_{13} \mathrm{H}_{19} \mathrm{O}_{3} \mathrm{Na}[\mathrm{M}+\mathrm{H}]^{+}:$223.1329; Found: 223.1329. Specific rotation: $[\alpha]^{\mathrm{D}}{ }_{24.6}$ +50 . $\left(\mathrm{c}=0.44 \mathrm{CH}_{3} \mathrm{OH}\right)$.

\section{Preparation of 25 from 16:}

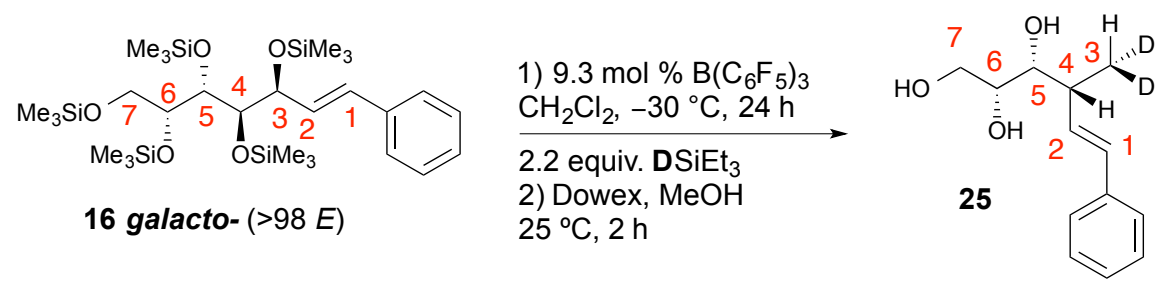

In a nitrogen-filled glovebox, a 1-dram vial was charged with $\mathrm{B}\left(\mathrm{C}_{6} \mathrm{~F}_{5}\right)_{3}(5.2 \mathrm{mg}, 0.010$ mmol, $9.3 \mathrm{~mol} \mathrm{\%})$ then triethylsilane- $d(35.0 \mu \mathrm{L}, 0.220 \mathrm{mmol}, 2.02$ equiv.) then dichloromethane $(0.5 \mathrm{~mL})$. A separate 1 -dram vial was charged with $\mathrm{Me}_{3} \mathrm{Si}$ styrylgalactose 16 (67.1 mg, $0.109 \mathrm{mmol}, 1.00$ equiv.) and a magnetic stir bar. The vials were sealed with a septum cap and removed from the glovebox. Both vials were allowed to cool to $-30{ }^{\circ} \mathrm{C}$ (dry ice/acetone bath) and the silane solution was transferred to the substrate vial by syringe under $\mathrm{N}_{2}$. The vial cap was wrapped in electrical tape and the vial was transferred to a $-30{ }^{\circ} \mathrm{C}$ cryobath and allowed to stir. After $24 \mathrm{~h}, \mathrm{NEt}_{3}(0.05 \mathrm{~mL})$ was added by syringe through the septa and the solution was allowed to slowly warm to $25{ }^{\circ} \mathrm{C}$. The stir bar was removed, rinsing with $\mathrm{CH}_{2} \mathrm{Cl}_{2}(0.2 \mathrm{~mL})$ and the quenched reaction mixture was concentrated under vacuum and then loaded onto an alumina plug (neutral, $10 \% \mathrm{H}_{2} \mathrm{O} ; 2 \times 1 \mathrm{~cm}$ ) with $100 \%$ hexanes $(1.5 \mathrm{~mL})$ then 95:5 hexanes:diethyl ether $(1.5 \mathrm{~mL})$, eluting with 95:5 hexanes:diethyl ether $(10 \mathrm{~mL})$ into a tared vial. The solution was concentrated under vacuum to afford a colorless oil ( $90 \%$ conv., $81.5 \mathrm{mg})$. Deprotection to alcohol was performed subsequently. The vial containing the silyl substrate was equipped with a stir bar and charged with Dowex ${ }^{\circledR}$ resin (10 beads). Methanol $(2.0 \mathrm{~mL})$ was added by syringe and the resulting solution was allowed to stir at $25{ }^{\circ} \mathrm{C}$. After $2 \mathrm{~h}$, the solution was passed through sand $(0.1 \mathrm{~cm})$, to remove the Dowex ${ }^{\circledR}$ beads, into a vial, rinsing with $100 \%$ methanol. The solution was concentrated to afford a white solid. Products were purified by silica gel chromatography ( 5 x $1 \mathrm{~cm}$ column) using gradient elution (100\% $\mathrm{CH}_{2} \mathrm{Cl}_{2}$ to $50: 1$ to $45: 1$ to $40: 1 \mathrm{CH}_{2} \mathrm{Cl}_{2}: \mathrm{MeOH}$ ) to afford 25 as a white solid (17.5 mg, $72 \%$ overall yield over 2 steps, $0.0778 \mathrm{mmol}$ ).

\section{Data for 25}




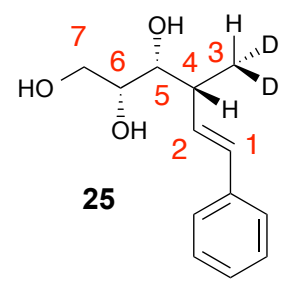

(2R,3R,4R,E)-4-(methyl- $\boldsymbol{d}_{\mathbf{2}}$ )-6-phenylhex-5-ene-1,2,3-triol (25): ${ }^{1} \mathrm{H}$ NMR (600 MHz, $\left.\mathrm{CD}_{3} \mathrm{OD}\right): \delta 7.39$ (d, $\left.J=7.2 \mathrm{~Hz}, 2 \mathrm{H}, \mathrm{Ph}\right), 7.28(\mathrm{t}, J=7.2 \mathrm{~Hz}, 2 \mathrm{H}, \mathrm{Ph}), 7.18(\mathrm{t}, J=7.8 \mathrm{~Hz}$, $1 \mathrm{H}, \mathrm{Ph}), 6.45\left(\mathrm{~d}, J_{\mathrm{H} 1 \mathrm{H} 2}=16.2 \mathrm{~Hz}, 1 \mathrm{H}, \mathrm{H}_{1}\right), 6.36\left(\mathrm{dd}, J_{\mathrm{H} 2, \mathrm{H} 1}=15.6 \mathrm{~Hz}, J_{\mathrm{H} 2, \mathrm{H} 3}=8.4 \mathrm{~Hz}\right.$, $\left.1 \mathrm{H}, \mathrm{H}_{2}\right), 3.70-3.66\left(\mathrm{~m}, 2 \mathrm{H}, \mathrm{H}_{6} \& \mathrm{H}_{7}\right), 3.63-3.60\left(\mathrm{~m}, 1 \mathrm{H}, \mathrm{H}_{7^{\prime}}\right), 3.49\left(\mathrm{dd}, J_{\mathrm{H} 5, \mathrm{H} 4}=6.0 \mathrm{~Hz}\right.$, $\left.J_{\mathrm{H} 5, \mathrm{H} 6}=3.6 \mathrm{~Hz}, 1 \mathrm{H}, \mathrm{H}_{5}\right), 2.60\left(\operatorname{app~q}, J_{\mathrm{H} 4, \mathrm{H} 5}=7.2 \mathrm{~Hz}, 1 \mathrm{H}, \mathrm{H}_{4}\right), 1.14\left(\mathrm{~d}, J_{\mathrm{H} 3, \mathrm{H} 4}=6.6 \mathrm{~Hz}\right.$, $\left.1 \mathrm{H}, \mathrm{H}_{3}\right) .{ }^{13} \mathrm{C}\left\{{ }^{1} \mathrm{H}\right\}$ NMR (151 MHz, $\left.\mathrm{CD}_{3} \mathrm{OD}\right): \delta 139.2(\mathrm{~s}, \mathrm{Ph}), 133.8\left(\mathrm{~s}, \mathrm{C}_{2}\right), 131.4\left(\mathrm{~s}, \mathrm{C}_{1}\right)$, $129.4(\mathrm{~s}, \mathrm{Ph}), 127.9(\mathrm{~s}, \mathrm{Ph}), 127.1(\mathrm{~s}, \mathrm{Ph}), 76.1\left(\mathrm{~s}, \mathrm{C}_{5}\right), 73.5\left(\mathrm{~s}, \mathrm{C}_{6}\right), 64.7\left(\mathrm{~s}, \mathrm{C}_{7}\right), 41.1(\mathrm{~s}$, $\mathrm{C}_{4}$ ), 17.5 (quintet, $J_{\mathrm{C}, \mathrm{D}}=19.6 \mathrm{~Hz}, \mathrm{C}_{3}$ ).

\section{Preparation of vinyl shift product 26 from 19:}

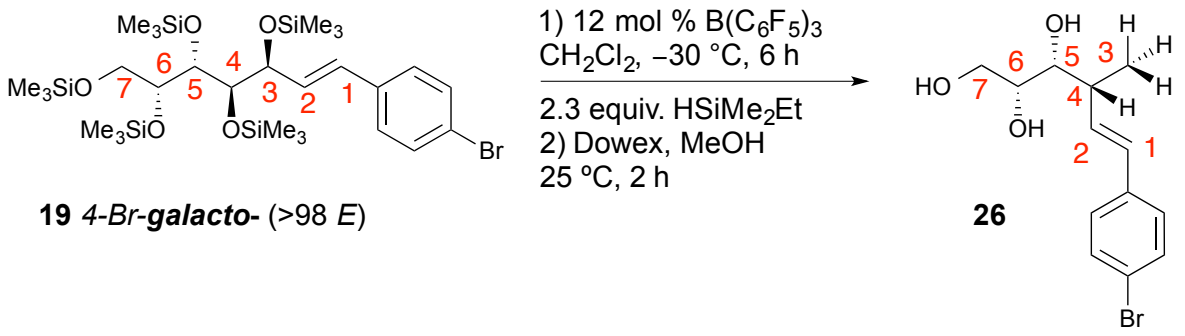

In a nitrogen-filled glovebox, a 1-dram vial was charged with $\mathrm{B}\left(\mathrm{C}_{6} \mathrm{~F}_{5}\right)_{3}(6.2 \mathrm{mg}, 0.012$ mmol, $12 \mathrm{~mol} \%)$ then dimethylethylsilane $(30.0 \mu \mathrm{L}, 0.227 \mathrm{mmol}, 2.31$ equiv.) then dichloromethane $(0.5 \mathrm{~mL})$. A separate 1-dram vial was charged with $\mathrm{Me}_{3} \mathrm{Si}-4-\mathrm{Br}$ styrylgalactose $19(68.2 \mathrm{mg}, 0.0983 \mathrm{mmol}, 1.00$ equiv.) and a magnetic stir bar. The vials were sealed with a septum cap and removed from the glovebox. Both vials were allowed to cool to $-30{ }^{\circ} \mathrm{C}$ (dry ice/acetone bath) and the silane solution was transferred to the substrate vial by syringe under $\mathrm{N}_{2}$. The vial cap was wrapped in electrical tape and the vial was transferred to a $-30{ }^{\circ} \mathrm{C}$ cryobath and allowed to stir. After $6 \mathrm{~h}, \mathrm{NEt}_{3}(0.05 \mathrm{~mL})$ was added by syringe through the septa and the solution was allowed to slowly warm to $25{ }^{\circ} \mathrm{C}$. The stir bar was removed, rinsing with $\mathrm{CH}_{2} \mathrm{Cl}_{2}(0.2 \mathrm{~mL})$ and the quenched reaction mixture was concentrated under vacuum and then loaded onto an alumina plug (neutral, $10 \% \mathrm{H}_{2} \mathrm{O} ; 2 \times 1 \mathrm{~cm}$ ) with $100 \%$ hexanes $(1.5 \mathrm{~mL})$ then $95: 5$ hexanes:diethyl ether $(1.5 \mathrm{~mL})$, eluting with 95:5 hexanes:diethyl ether $(10 \mathrm{~mL})$ into a tared vial. The solution was concentrated under vacuum to afford a colorless oil (79.7 mg). Deprotection to alcohol was performed subsequently. The vial containing the silyl substrate was equipped with a stir bar and charged with Dowex ${ }^{\circledR}$ resin (10 beads). Methanol (2.0 mL) was added by syringe and the resulting solution was allowed to stir at $25^{\circ} \mathrm{C}$. After $2 \mathrm{~h}$, 
the solution was passed through sand $(0.1 \mathrm{~cm})$ to remove the Dowex ${ }^{\circledR}$ beads into a vial, rinsing with $100 \%$ methanol. The solution was concentrated to afford a white solid. Products were purified by silica gel chromatography ( 4 x $1 \mathrm{~cm}$ column) using gradient elution ( $100 \% \mathrm{CH}_{2} \mathrm{Cl}_{2}$ to $60: 1$ to $50: 1$ to $49: 1$ to $48: 1$ to $47: 1$ to $\left.46: 1 \mathrm{CH}_{2} \mathrm{Cl}_{2}: \mathrm{MeOH}\right)$ to afford $(2 R, 3 R, 4 R, E)$-6-(4-bromophenyl)-4-methylhex-5-ene-1,2,3-triol $\mathbf{2 6}$ as a white solid. A single crystal was obtained by recrystallization from $\mathrm{CH}_{2} \mathrm{Cl}_{2} / \mathrm{MeOH}$ by slow evaporation at room temperature under neutral atmosphere. Note only the $E$ isomer was crystalline.

\section{Data for 26}

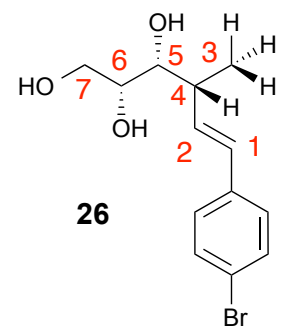

(2R,3R,4R,E)-6-(4-bromophenyl)-4-methylhex-5-ene-1,2,3-triol (26): Data from the fraction that was recrystallized; a $67: 33 \mathrm{E} / \mathrm{Z}$ mixture. ${ }^{1} \mathrm{H} \mathrm{NMR}\left(600 \mathrm{MHz}, \mathrm{CD}_{3} \mathrm{OD}\right): \delta$ 7.48 (d, $J=7.8 \mathrm{~Hz}, 2 \mathrm{H}, \mathrm{Ar}, Z), 7.44$ (d, $J=8.4 \mathrm{~Hz}, 2 \mathrm{H}, \mathrm{Ar}, E), 7.32$ (d, $J=8.4 \mathrm{~Hz}, 2 \mathrm{H}$, Ar, E), 7.29 (d, $J=8.4 \mathrm{~Hz}, 2 \mathrm{H}, \mathrm{Ar}, Z), 6.45\left(\mathrm{~d}, J_{\mathrm{H} 1, \mathrm{H} 2}=12.0 \mathrm{~Hz}, 1 \mathrm{H}, \mathrm{H}_{1}, Z\right), 6.44-6.35$ $\left(\mathrm{m}, 2 \mathrm{H}, \mathrm{H}_{1} \& \mathrm{H}_{2}, E\right), 5.75\left(\mathrm{dd}, J_{\mathrm{H} 2, \mathrm{H} 1}=11.4 \mathrm{~Hz}, J_{\mathrm{H} 2, \mathrm{H} 4}=10.8 \mathrm{~Hz}, 1 \mathrm{H}, \mathrm{H}_{2}, Z\right), 3.69-3.64$ $\left(\mathrm{m}, 2 \mathrm{H}, \mathrm{H}_{6} \& \mathrm{H}_{7}, E\right), 3.63-3.58\left(\mathrm{~m}, 2 \mathrm{H}, \mathrm{H}_{7}, E, \& \mathrm{H}_{6}, Z\right), 3.51$ (dd, $J_{\mathrm{H} 7, \mathrm{H} 7}=10.8 \mathrm{~Hz}$, $\left.J_{\mathrm{H} 7, \mathrm{H} 6}=4.8 \mathrm{~Hz}, 1 \mathrm{H}, \mathrm{H}_{7}\right), 3.48\left(\mathrm{dd}, J_{\mathrm{H} 5, \mathrm{H} 4}=6.0 \mathrm{~Hz}, J_{\mathrm{H} 5, \mathrm{H} 6}=3.6 \mathrm{~Hz}, 1 \mathrm{H}, \mathrm{H}_{5}, E\right), 3.44(\mathrm{dd}$,

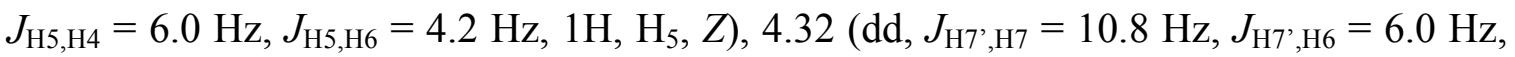
$\left.1 \mathrm{H}, \mathrm{H}_{7}, Z\right), 3.07-3.01\left(\mathrm{~m}, 1 \mathrm{H}, \mathrm{H}_{4}, Z\right), 2.64-2.58\left(\mathrm{~m}, 1 \mathrm{H}, \mathrm{H}_{4}, E\right), 1.17\left(\mathrm{~d}, J_{\mathrm{H} 3, \mathrm{H} 4}=6.6 \mathrm{~Hz}\right.$, $\left.3 \mathrm{H}, \mathrm{H}_{3}, E\right), 1.12\left(\mathrm{~d}, J_{\mathrm{H} 4, \mathrm{H} 3}=6.6 \mathrm{~Hz}, 3 \mathrm{H}, \mathrm{H}_{3}, Z\right) .{ }^{13} \mathrm{C}\left\{{ }^{1} \mathrm{H}\right\} \mathrm{NMR}\left(151 \mathrm{MHz}, \mathrm{CD}_{3} \mathrm{OD}\right): \delta$ 138.4 (s, Ar), 138.1 (s, Ar), 137.1 (s, C $2, Z$ ), 135.1 (s, $\left.\mathrm{C}_{2}, E\right), 132.5$ (s, Ar), 132.4 (s, Ar), 131.6 (s, Ar), 130.1 (s, C $1, E), 129.0\left(\mathrm{~s}, \mathrm{C}_{1}, Z\right), 128.9$ (s, Ar), 121.4 (s, Ar), 76.1 (s, $\mathrm{C}_{5}$, $E), 76.0\left(\mathrm{~s}, \mathrm{C}_{5}, Z\right), 73.5$ (s, $\left.\mathrm{C}_{6}, E\right), 73.4\left(\mathrm{~s}, \mathrm{C}_{6}, Z\right), 64.7$ (s, $\left.\mathrm{C}_{7}, E\right), 64.6$ (s, $\left.\mathrm{C}_{7}, Z\right), 41.3$ (s, $\left.\mathrm{C}_{4}, E\right), 36.0\left(\mathrm{~s}, \mathrm{C}_{4}, Z\right), 18.1\left(\mathrm{~s}, \mathrm{C}_{3}, Z\right), 17.9\left(\mathrm{~s}, \mathrm{C}_{3}, E\right)$. (*Note: One Ar carbon is overlapping for $E / Z$ ).

\section{Scale-up preparation of vinyl shift product 27 from 16:}

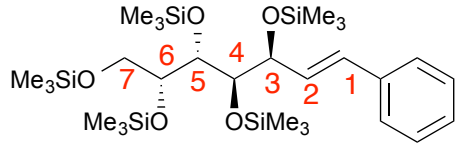

16-galacto- (>98 E)
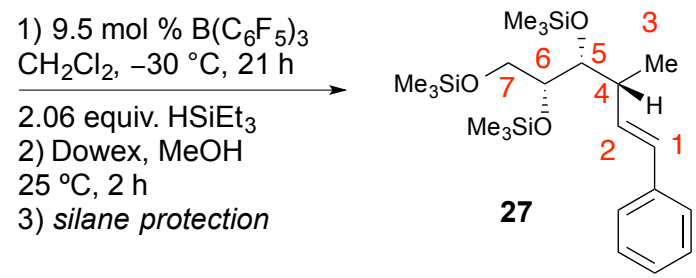
In a nitrogen-filled glovebox, a 20 -mL scintillation vial was charged with TMSstyrylgalactose 16 ( $329.2 \mathrm{mg}, 0.535 \mathrm{mmol}, 1.00$ equiv.) and a magnetic stir bar. A 1-dram vial was charged with $\mathrm{B}\left(\mathrm{C}_{6} \mathrm{~F}_{5}\right)_{3}(25.9 \mathrm{mg}, 0.0505 \mathrm{mmol}, 0.095$ equiv.), triethylsilane (175.8 $\mu \mathrm{L}, 1.10 \mathrm{mmol}, 2.06$ equiv.), and $\mathrm{CH}_{2} \mathrm{Cl}_{2}(2.5 \mathrm{~mL})$. The 1 dram vial was sealed with a septa cap and the scintillation vial was sealed with a septa and wrapped in electrical tape and both were removed from the glovebox. The vials were allowed to cool to $-30{ }^{\circ} \mathrm{C}$ (dry ice/acetone bath). The $\mathrm{B}\left(\mathrm{C}_{6} \mathrm{~F}_{5}\right)_{3}$ solution was transferred to the substrate

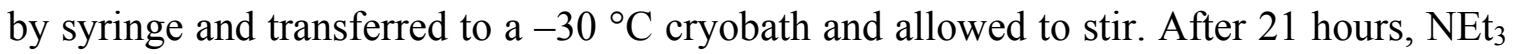
$(0.15 \mathrm{~mL})$ was added by syringe through the septa and the stir bar was removed, rinsing with $\mathrm{CH}_{2} \mathrm{Cl}_{2}(0.2 \mathrm{~mL})$. The quenched reaction mixture was concentrated under vacuum and then loaded onto an alumina plug (neutral, $10 \% \mathrm{H}_{2} \mathrm{O} ; 2 \times 1 \mathrm{~cm}$ ) with $100 \%$ hexanes $(1.5 \mathrm{~mL})$ then $95: 5$ hexanes:diethyl ether $(1.5 \mathrm{~mL})$, eluting with 95:5 hexanes:diethyl ether $(10 \mathrm{~mL})$ into a tared vial. The solution was concentrated under vacuum to afford a colorless oil (353.8 mg). Deprotection was subsequently performed. The vial containing the silyl substrate was equipped with a stir bar and charged with Dowex ${ }^{\circledR}$ resin $(\sim 30$ beads). Methanol $(4.0 \mathrm{~mL})$ was added by syringe and the resulting solution was allowed to stir at $22{ }^{\circ} \mathrm{C}$. After $2 \mathrm{~h}$, the solution was passed through sand $(0.1 \mathrm{~cm})$ to remove the Dowex ${ }^{\circledR}$ beads into a tared vial, rinsing with $100 \%$ methanol. The solution was concentrated by vacuum to afford a colorless oil $(141.6 \mathrm{mg})$. The product was purified by silica gel chromatography ( 4.5 " $\mathrm{x} 0.5$ " column) using gradient elution $\left(100 \% \mathrm{CH}_{2} \mathrm{Cl}_{2}\right.$ to $50: 1$ to $45: 1$ to $40: 1$ to $39: 1$ to $38: 1$ to $\left.37: 1 \mathrm{CH}_{2} \mathrm{Cl}_{2}: \mathrm{MeOH}\right)$ to afford $(2 R, 3 R, 4 R, E)-4-$ methyl-6-phenylhex-5-ene-1,2,3-triol $\mathbf{2 4}$ as a white solid (105.3 $\mathrm{mg}$, 89\% overall yield over 2 steps, $0.474 \mathrm{mmol})$.

Silyl protection was performed subsequently. The vial containing triol substrate was charged with a stir bar, equipped with a septum and purged with $\mathrm{N}_{2}$ for 5 minutes. Dichloromethane $(2.5 \mathrm{~mL})$ was added by syringe followed by pyridine $(172 \mu \mathrm{L}, 2.13$ mmol, 4.50 equiv) and chlorotrimethylsilane $\left(210 \mu \mathrm{L}, 1.66 \mathrm{mmol}, 3.50\right.$ equiv) at $22{ }^{\circ} \mathrm{C}$. The resulting turbid solution was allowed to stir for 5 hours before quenching with deionized water $(2.5 \mathrm{~mL})$. Diethyl ether $(5.0 \mathrm{~mL})$ was added and the layers were separated. The aqueous was washed with $\mathrm{Et}_{2} \mathrm{O}(3 \times 5 \mathrm{~mL})$ and the combined organic layers were passed through a plug of sodium sulfate $(5 \times 1 \mathrm{~cm})$, and concentrated under vacuum. Toluene $(3 \times 10 \mathrm{~mL})$ to remove residual pyridine was added and removed by vacuum to afford a colorless oil $(155.5 \mathrm{mg})$. The product was purified by alumina column $\left(10 \% \mathrm{H}_{2} \mathrm{O}, 2 \times 1 \mathrm{~cm}\right)$ eluting with $100 \%$ hexanes to afford $\mathrm{Me}_{3} \mathrm{Si}-(2 R, 3 R, 4 R, E)-4-$ methyl-6-phenylhex-5-ene-1,2,3-triol 27 as a colorless oil (137.2 mg, 66\% yield, 0.313 mmol).

\section{Data for 27}




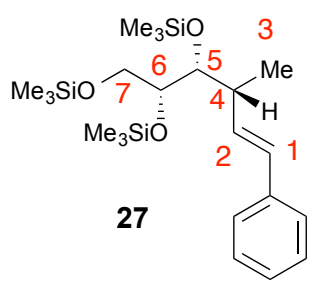

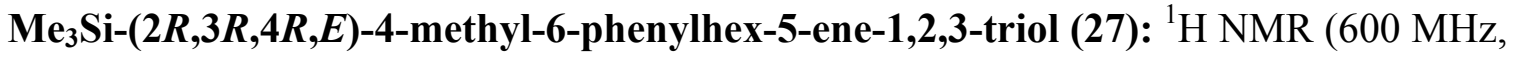
$\left.\mathrm{CDCl}_{3}\right): \delta 7.34(\mathrm{~d}, J=7.8 \mathrm{~Hz}, 2 \mathrm{H}, \mathrm{Ph}), 7.29(\mathrm{t}, J=7.2 \mathrm{~Hz}, 2 \mathrm{H}, \mathrm{Ph}), 7.19(\mathrm{t}, J=7.2 \mathrm{~Hz}$, $1 \mathrm{H}, \mathrm{Ph}), 6.38\left(\mathrm{~d}, J_{\mathrm{H} 1, \mathrm{H} 2}=16.2 \mathrm{~Hz}, 1 \mathrm{H}, \mathrm{H}_{1}\right), 6.20\left(\mathrm{dd}, J_{\mathrm{H} 2, \mathrm{H} 1}=16.2 \mathrm{~Hz}, J_{\mathrm{H} 2, \mathrm{H} 4}=9.0 \mathrm{~Hz}\right.$, $\left.1 \mathrm{H}, \mathrm{H}_{2}\right), 3.73\left(\mathrm{dt}, J_{\mathrm{H} 6, \mathrm{H} 5}=7.2 \mathrm{~Hz}, J_{\mathrm{H} 6, \mathrm{H} 7}=4.2 \mathrm{~Hz}, 1 \mathrm{H}, \mathrm{H}_{6}\right), 3.60\left(\mathrm{dd}, J_{\mathrm{H} 7, \mathrm{H} 7}=10.2 \mathrm{~Hz}\right.$, $\left.J_{\mathrm{H} 7, \mathrm{H} 6}=4.2 \mathrm{~Hz}, 1 \mathrm{H}, \mathrm{H}_{7}\right), 3.58\left(\mathrm{dd}, J_{\mathrm{H} 5, \mathrm{H} 6}=6.6 \mathrm{~Hz}, J_{\mathrm{H} 5, \mathrm{H} 4}=3.6 \mathrm{~Hz}, 1 \mathrm{H}, \mathrm{H}_{5}\right), 3.55(\mathrm{dd}$, $\left.J_{\mathrm{H} 7^{\prime}, \mathrm{H} 7}=10.2 \mathrm{~Hz}, J_{\mathrm{H}^{\prime}, \mathrm{H} 6}=6.6 \mathrm{~Hz}, 1 \mathrm{H}, \mathrm{H}_{7^{\prime}}\right), 2.65$ (sextet, $7.2 \mathrm{~Hz}, J_{\mathrm{H} 4, \mathrm{H} 3}=J_{\mathrm{H} 4, \mathrm{H} 5}=7.2 \mathrm{~Hz}$, $\left.1 \mathrm{H}, \mathrm{H}_{4}\right), 1.08\left(\mathrm{~d}, J_{\mathrm{H} 3, \mathrm{H} 4}=6.6 \mathrm{~Hz}, 3 \mathrm{H}, \mathrm{H}_{3}\right), 0.15\left(\mathrm{~s}, 9 \mathrm{H}, \mathrm{Si}\left(\mathrm{CH}_{3}\right)_{3}\right), 0.10\left(\mathrm{~s}, 9 \mathrm{H}, \mathrm{Si}\left(\mathrm{CH}_{3}\right)_{3}\right)$, 0.09 (s, 9H, $\left.\mathrm{Si}\left(\mathrm{CH}_{3}\right)_{3}\right) .{ }^{13} \mathrm{C}\left\{{ }^{1} \mathrm{H}\right\} \mathrm{NMR}\left(151 \mathrm{MHz}, \mathrm{CDCl}_{3}\right): \delta 138.0(\mathrm{~s}, \mathrm{Ph}), 134.2\left(\mathrm{~s}, \mathrm{C}_{2}\right)$, $129.9\left(\mathrm{~s}, \mathrm{C}_{1}\right), 128.6(\mathrm{~s}, \mathrm{Ph}), 126.9(\mathrm{~s}, \mathrm{Ph}), 126.1(\mathrm{~s}, \mathrm{Ph}), 77.7\left(\mathrm{~s}, \mathrm{C}_{5}\right), 75.4\left(\mathrm{~s}, \mathrm{C}_{6}\right), 64.3$ (s, $\left.\mathrm{C}_{7}\right), 39.8\left(\mathrm{~s}, \mathrm{C}_{4}\right), 18.3\left(\mathrm{~s}, \mathrm{C}_{3}\right), 1.0\left(\mathrm{~s}, \mathrm{Si}\left(\mathrm{CH}_{3}\right)_{3}\right), 0.9\left(\mathrm{~s}, \mathrm{Si}\left(\mathrm{CH}_{3}\right)_{3}\right),-0.3\left(\mathrm{~s}, \mathrm{Si}\left(\mathrm{CH}_{3}\right)_{3}\right)$.

\section{Preparation of carbocycle product 20 from vinyl shifted product 27:}
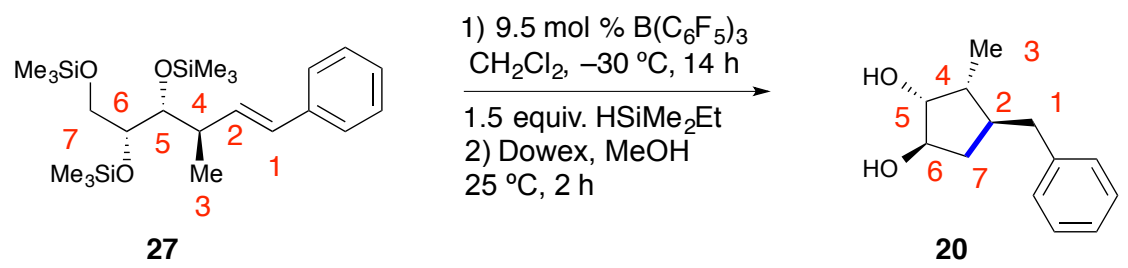

In a nitrogen-filled glovebox, a 1-dram vial was charged with $\mathrm{Me}_{3} \mathrm{Si}-(2 R, 3 R, 4 R, E)-4-$ methyl-6-phenylhex-5-ene-1,2,3-triol 27 (45.0 mg, $0.103 \mathrm{mmol}, 1.00$ equiv.) and a magnetic stir bar. A separate 1-dram vial was charged with $\mathrm{B}\left(\mathrm{C}_{6} \mathrm{~F}_{5}\right)_{3}(5.0 \mathrm{mg}, 0.0098$ mmol, 0.095 equiv.), dimethylethylsilane (19.8 $\mu \mathrm{L}, 0.150 \mathrm{mmol}, 1.46$ equiv.) and $\mathrm{CH}_{2} \mathrm{Cl}_{2}$ $(0.5 \mathrm{~mL})$. The vials were sealed with a septa cap and removed from the glovebox. The vials were allowed to cool to $-30{ }^{\circ} \mathrm{C}$ (dry ice/acetone bath). The $\mathrm{B}\left(\mathrm{C}_{6} \mathrm{~F}_{5}\right)_{3}$ solution was transferred to the substrate by syringe and allowed to stir. The vial was wrapped with electrical tape and transferred to a $-30{ }^{\circ} \mathrm{C}$ cryobath and allowed to stir. After 14 hours, $\mathrm{NEt}_{3}(0.05 \mathrm{~mL})$ was added by syringe through the septa and the stir bar was removed, rinsing with $\mathrm{CH}_{2} \mathrm{Cl}_{2}(2 \mathrm{~mL})$. The quenched reaction mixture was concentrated under vacuum and then loaded onto an alumina plug (neutral, $10 \% \mathrm{H}_{2} \mathrm{O} ; 2 \times 1 \mathrm{~cm}$ ) with $100 \%$ hexanes $(1.5 \mathrm{~mL})$ then 95:5 hexanes:diethyl ether $(1.5 \mathrm{~mL})$, eluting with 95:5 hexanes:diethyl ether $(10 \mathrm{~mL})$ into a tared vial. The solution was concentrated under vacuum to afford a colorless oil $(46.9 \mathrm{mg})$. The vial containing the silyl substrate was equipped with a stir bar and charged with Dowex ${ }^{\circledR}$ resin $(\sim 10$ beads $)$. Methanol $(2.0 \mathrm{~mL})$ was added by syringe and the resulting solution was allowed to stir at $25{ }^{\circ} \mathrm{C}$. After $2 \mathrm{~h}$, the solution was passed through sand $(0.1 \mathrm{~cm})$ to remove the Dowex ${ }^{\circledR}$ beads into a tared 
vial, rinsing with $100 \%$ methanol and concentrated to afford a colorless oil $(20.6 \mathrm{mg})$. Products were purified by silica gel chromatography $(5 \times 1 \mathrm{~cm}$ column) using gradient elution (100\% $\mathrm{CH}_{2} \mathrm{Cl}_{2}$ to $50: 1$ to $49: 1$ to $\left.48: 1 \mathrm{CH}_{2} \mathrm{Cl}_{2}: \mathrm{MeOH}\right)$ to afford carbocycle from $\mathrm{Me}_{3} \mathrm{Si}$-styrylgalactose 20 as a white solid (17.6 $\mathrm{mg}, 83 \%$ yield, $0.0853 \mathrm{mmol}$ ).

\section{Data for 20}

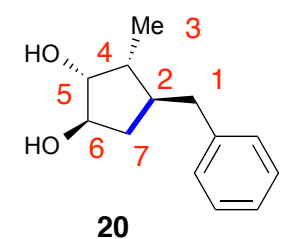

(1R,2R,3R,4S)-4-benzyl-3-methylcyclopentane-1,2-diol (20): ${ }^{1} \mathrm{H}$ NMR $(600 \mathrm{MHz}$, $\left.\mathrm{CD}_{3} \mathrm{OD}\right): \delta 7.26(\mathrm{t}, J=7.8 \mathrm{~Hz}, 2 \mathrm{H}, \mathrm{Ph}), 7.19(\mathrm{~d}, J=7.2 \mathrm{~Hz}, 2 \mathrm{H}, \mathrm{Ph}), 7.16(\mathrm{t}, J=7.2 \mathrm{~Hz}$, $1 \mathrm{H}, \mathrm{Ph}), 3.92\left(\mathrm{ddd}, J_{\mathrm{H} 6, \mathrm{H} 7}=6.6 \mathrm{~Hz}, J_{\mathrm{H} 6, \mathrm{H} 7}=4.2 \mathrm{~Hz}, J_{\mathrm{H} 6, \mathrm{H} 5}=2.4 \mathrm{~Hz}, 1 \mathrm{H}, \mathrm{H}_{6}\right), 3.81(\mathrm{dd}$, $\left.J_{\mathrm{H} 5, \mathrm{H} 4}=4.8 \mathrm{~Hz}, J_{\mathrm{H} 5, \mathrm{H} 6}=2.4 \mathrm{~Hz}, 1 \mathrm{H}, \mathrm{H}_{5}\right), 2.88\left(\mathrm{dd}, J_{\mathrm{H} 1, \mathrm{H} 1}=13.2 \mathrm{~Hz}, J_{\mathrm{H} 1, \mathrm{H} 2}=5.4 \mathrm{~Hz}, 1 \mathrm{H}\right.$, $\left.\mathrm{H}_{1}\right), 2.47\left(\mathrm{dd}, J_{\mathrm{H} 1^{\prime}, \mathrm{H} 1}=13.2 \mathrm{~Hz}, J_{\mathrm{Hl}^{\prime}, \mathrm{H} 2}=9.0 \mathrm{~Hz}, 1 \mathrm{H}, \mathrm{H}_{1}{ }^{\prime}\right), 2.09\left(\mathrm{ddd}, J_{\mathrm{H} 7, \mathrm{H} 7^{\prime}}=13.8 \mathrm{~Hz}\right.$, $\left.J_{\mathrm{H} 7, \mathrm{H} 2}=8.4 \mathrm{~Hz}, J_{\mathrm{H} 7, \mathrm{H} 6}=6.6 \mathrm{~Hz}, 1 \mathrm{H}, \mathrm{H}_{7}\right), 1.96-1.89\left(\mathrm{~m}, 1 \mathrm{H}, \mathrm{H}_{2}\right), 1.89-1.83\left(\mathrm{~m}, 1 \mathrm{H}, \mathrm{H}_{4}\right)$,

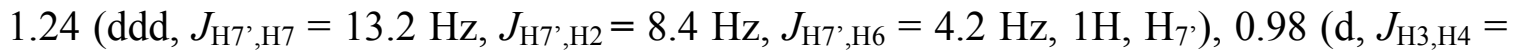
$\left.7.2 \mathrm{~Hz}, 3 \mathrm{H}, \mathrm{H}_{3}\right) .{ }^{13} \mathrm{C}\left\{{ }^{1} \mathrm{H}\right\}$ NMR (151 MHz, $\left.\mathrm{CD}_{3} \mathrm{OD}\right): \delta 142.9(\mathrm{~s}, \mathrm{Ph}), 129.9(\mathrm{~s}, \mathrm{Ph}), 129.2$ $(\mathrm{s}, \mathrm{Ph}), 126.8(\mathrm{~s}, \mathrm{Ph}), 82.4\left(\mathrm{~s}, \mathrm{C}_{5}\right), 78.8\left(\mathrm{~s}, \mathrm{C}_{6}\right), 45.6\left(\mathrm{~s}, \mathrm{C}_{4}\right), 43.3\left(\mathrm{~s}, \mathrm{C}_{2}\right), 42.7\left(\mathrm{~s}, \mathrm{C}_{1}\right), 39.6$ $\left(\mathrm{s}, \mathrm{C}_{7}\right), 13.1\left(\mathrm{~s}, \mathrm{C}_{3}\right)$. HRMS $\left(\mathrm{ESI}^{+}\right)$: Calcd for $\mathrm{C}_{13} \mathrm{H}_{18} \mathrm{O}_{2} \mathrm{Na}[\mathrm{M}+\mathrm{Na}]^{+}:$229.1199; Found: 229.1200. Specific rotation: $[\alpha]^{\mathrm{D}} 24.9+58 .\left(\mathrm{c}=0.42 \mathrm{CH}_{3} \mathrm{OH}\right)$.

\section{Preparation of cyclic product 28 by $\left[\mathrm{Ph}_{3} \mathrm{C}\right]\left[\mathrm{B}\left(\mathrm{C}_{6} \mathrm{~F}_{5}\right)_{4}\right]$ cyclization:}

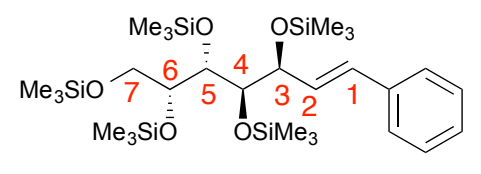

16 galacto- $(>98 E)$

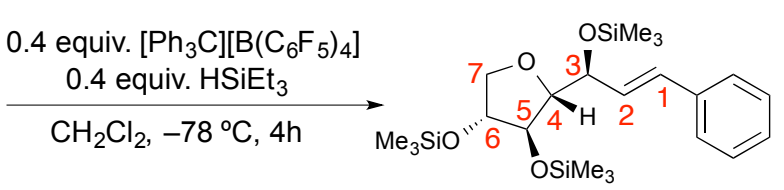

28

In a nitrogen-filled glovebox, a 1-dram vial was charged with TMS-styrylgalactose 16 (329.2 mg, $0.101 \mathrm{mmol}, 1.00$ equiv.), a magnetic stir bar, and $\left[\mathrm{Ph}_{3} \mathrm{C}\right]\left[\mathrm{B}\left(\mathrm{C}_{6} \mathrm{~F}_{5}\right)_{4}\right](34.3 \mathrm{mg}$, $0.0372 \mathrm{mmol}, 0.368$ equiv.). A second 1-dram vial was charged with triethylsilane (6.5 $\mu \mathrm{L}, 0.376 \mathrm{mmol}, 0.372$ equiv.) and $\mathrm{CH}_{2} \mathrm{Cl}_{2}(0.5 \mathrm{~mL})$. The 1 -dram vials were sealed with a septa cap and then and wrapped in electrical tape and both were removed from the glovebox. The vials were allowed to cool to $-78{ }^{\circ} \mathrm{C}$ (dry ice/acetone bath). The silane solution was transferred to the substrate by syringe and transferred to held at $-78^{\circ} \mathrm{C}$ and allowed to stir. After 4 hours, $\mathrm{NEt}_{3}(0.10 \mathrm{~mL})$ was added by syringe through the septa and the stir bar was removed, rinsing with $\mathrm{CH}_{2} \mathrm{Cl}_{2}(0.2 \mathrm{~mL})$. The quenched reaction mixture was concentrated under vacuum to yield lightly yellow oil. Products were purified by alumina gel chromatography (neutral, $10 \% \mathrm{H}_{2} \mathrm{O} ; 5 \times 1 \mathrm{~cm}$ ) using gradient 
elution (100\% hexanes to $50: 1$ to $30: 1$ to $20: 1$ hexanes:diethyl ether) to afford $\mathbf{2 8}$ as a colorless oil ( $11.7 \mathrm{mg}, 26.0 \%$ overall yield, $0.0258 \mathrm{mmol})$. Deprotection to give the free alcohol (NMR spectra provided) product was also performed following standard Dowex ${ }^{\circledR}$ deprotection vide supra.

\section{Data for 28}

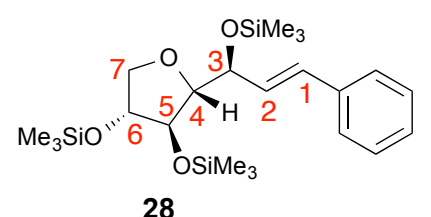

(((2R,3R,4R)-2-((S,E)-3-phenyl-1-((trimethylsilyl)oxy)allyl)tetrahydrofuran-3,4diyl)bis(oxy))bis(trimethylsilane) (28): ${ }^{1} \mathrm{H}$ NMR $\left(600 \mathrm{MHz}, \mathrm{CDCl}_{3}\right): \delta 7.38(\mathrm{~d}, J=7.2$ $\mathrm{Hz}, 2 \mathrm{H}, \mathrm{Ar}$ ), 7.31 (t, $J=7.2 \mathrm{~Hz}, 2 \mathrm{H}, \mathrm{Ar}$ ), 7.23 (t, $J=7.2 \mathrm{~Hz}, 1 \mathrm{H}, \mathrm{Ar}$ ), 6.55 (d, $J=16.2$ $\left.\mathrm{Hz}, 1 \mathrm{H}, \mathrm{H}_{1}\right), 6.27\left(\mathrm{dd}, J_{\mathrm{H} 2, \mathrm{H} 1}=16.2 \mathrm{~Hz}, J_{\mathrm{H} 2, \mathrm{H} 3}=6.6 \mathrm{~Hz}, 1 \mathrm{H}, \mathrm{H}_{2}\right), 4.44\left(\mathrm{ddd}, J_{\mathrm{H} 3, \mathrm{H} 4}=7.8\right.$ $\left.\mathrm{Hz}, J_{\mathrm{H} 3, \mathrm{H} 2}=6.6 \mathrm{~Hz}, J_{\mathrm{H} 3, \mathrm{H} 1}=1.2 \mathrm{~Hz}, 1 \mathrm{H}, \mathrm{H}_{3}\right), 4.05-4.03\left(\mathrm{~m}, 2 \mathrm{H}, \mathrm{H}_{5} \& \mathrm{H}_{6}\right), 3.96(\mathrm{dd}$, $\left.J_{\mathrm{H} 7, \mathrm{H} 7}=9.6 \mathrm{~Hz}, J_{\mathrm{H} 7, \mathrm{H} 6}=3.6 \mathrm{~Hz}, 1 \mathrm{H}, \mathrm{H}_{7}\right), 3.74-3.71\left(\mathrm{~m}, 2 \mathrm{H}, \mathrm{H}_{7}, \& \mathrm{H}_{4}\right), 0.15(\mathrm{~s}, 9 \mathrm{H},-$ $\mathrm{SiCH}_{3}$ ), 0.10 (s, 9H, $\left.-\mathrm{SiCH}_{3}\right), 0.09$ (s, 9H, $\left.-\mathrm{SiCH}_{3}\right) .{ }^{13} \mathrm{C}\left\{{ }^{1} \mathrm{H}\right\} \mathrm{NMR}\left(151 \mathrm{MHz}, \mathrm{CDCl}_{3}\right): \delta$ 137.1 (s, Ar), 131.5 (s, $\mathrm{C}_{1}$ ), 129.8 (s, $\mathrm{C}_{2}$ ), 128.7 (s, Ar), 127.6 (s, Ar), 126.7 (s, Ar), 89.0 $\left(\mathrm{s}, \mathrm{C}_{4}\right), 79.4\left(\mathrm{~s}, \mathrm{C}_{5 \text { or }}\right), 78.9$ (s, $\left.\mathrm{C}_{5 \text { or } 6}\right), 74.0\left(\mathrm{~s}, \mathrm{C}_{3}\right), 73.6\left(\mathrm{~s}, \mathrm{C}_{7}\right), 0.6,0.4,0.2$ (each a s, $\left.\mathrm{Si}\left(\mathrm{CH}_{3}\right)_{3}\right)$.

\section{Preparation of vinyl shift product 24 from 28:}

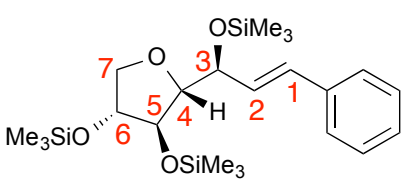

28

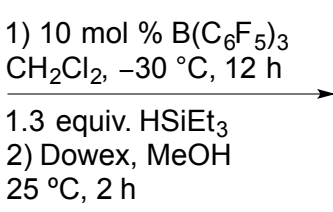

$25^{\circ} \mathrm{C}, 2 \mathrm{~h}$

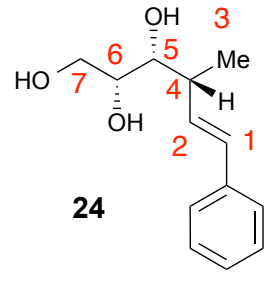

In a nitrogen-filled glovebox, a 1-dram vial was charged with $\mathrm{B}\left(\mathrm{C}_{6} \mathrm{~F}_{5}\right)_{3}(2.6 \mathrm{mg}, 0.0026$ mmol, $10 \mathrm{~mol} \%)$ then triethylsilane $(5.3 \mu \mathrm{L}, 0.034 \mathrm{mmol}, 1.3$ equiv.) and then dichloromethane $(0.250 \mathrm{~mL})$. A separate 1-dram vial was charged with furan cyclized $\mathrm{Me}_{3} \mathrm{Si}$-styrylgalactose 28 (11.7 mg, $0.026 \mathrm{mmol}, 1.0$ equiv.) and a magnetic stir bar. The vials were sealed with a septum cap and removed from the glovebox. Both vials were allowed to cool to $-30{ }^{\circ} \mathrm{C}$ (dry ice/acetone bath) and the silane solution was transferred to the substrate vial by syringe under $\mathrm{N}_{2}$. The vial cap was wrapped in electrical tape and the vial was transferred to a $-30{ }^{\circ} \mathrm{C}$ cryobath and allowed to stir. After $12 \mathrm{~h}, \mathrm{NEt}_{3}(0.05$ $\mathrm{mL}$ ) was added by syringe through the septa and the solution was allowed to slowly warm to $25{ }^{\circ} \mathrm{C}$. The stir bar was removed, rinsing with $\mathrm{CH}_{2} \mathrm{Cl}_{2}(0.2 \mathrm{~mL})$ and the quenched reaction mixture was concentrated under vacuum and then loaded onto an 
alumina plug (neutral, $10 \% \mathrm{H}_{2} \mathrm{O} ; 2 \times 1 \mathrm{~cm}$ ) with $100 \%$ hexanes $(1.5 \mathrm{~mL}$ ) then $95: 5$ hexanes:diethyl ether $(1.5 \mathrm{~mL})$, eluting with 95:5 hexanes:diethyl ether $(10 \mathrm{~mL})$ into a tared vial. The solution was concentrated under vacuum to afford a colorless oil (31.5 $\mathrm{mg}$ ). Deprotection to alcohol was performed subsequently. The vial containing the silyl substrate was equipped with a stir bar and charged with Dowex ${ }^{\circledR}$ resin (10 beads). Methanol $(2.0 \mathrm{~mL})$ was added by syringe and the resulting solution was allowed to stir at $25^{\circ} \mathrm{C}$. After $2 \mathrm{~h}$, the solution was passed through sand $(0.1 \mathrm{~cm})$ to remove the Dowex ${ }^{\circledR}$ beads into a vial, rinsing with $100 \%$ methanol. The solution was concentrated to afford a white solid. Products were purified by silica gel chromatography ( 4 x $1 \mathrm{~cm}$ column) using gradient elution ( $100 \% \mathrm{CH}_{2} \mathrm{Cl}_{2}$ to $50: 1$ to $45: 1$ to $40: 1$ to $\left.35: 1 \mathrm{CH}_{2} \mathrm{Cl}_{2}: \mathrm{MeOH}\right)$ to afford $(2 R, 3 R, 4 R, E)$-4-methyl-6-phenylhex-5-ene-1,2,3-triol 24. Data matched that from the same product derived from 16 vide supra.

\section{Crystallographic Data for derivative of 26 for stereochemical determination:}

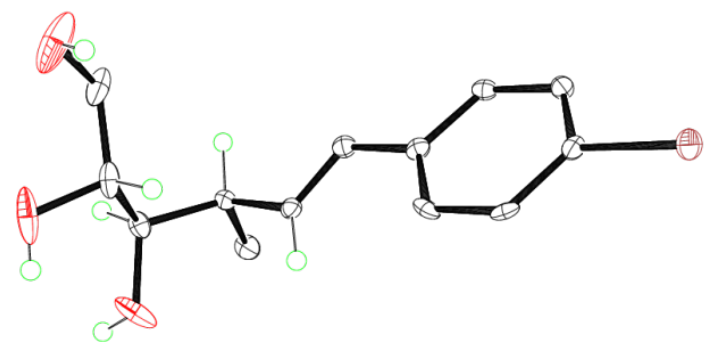

ORTEP representation of the solid state molecular structure of $\mathbf{2 6}$ derivative; ellipsoids drawn at $50 \%$ probability, the majority of hydrogen atoms are omitted for clarity.

\begin{tabular}{|c|c|c|c|}
\hline Empirical formula & $\mathrm{C}_{13} \mathrm{H}_{17} \mathrm{BrO}_{3}$ & $\mathrm{D}_{\text {calcd }}\left(\mathrm{Mg} \mathrm{m}^{-3}\right)$ & 1.579 \\
\hline$F_{\mathrm{w}}$ & 301.17 & Radiation & $\begin{array}{l}\mathrm{Cu}_{\mathrm{K} a}, \lambda= \\
1.54178 \AA\end{array}$ \\
\hline Colour, habit & Colourless, Plate & $\begin{array}{l}\text { Absorption coeff. }(\mu) \\
\left(\mathrm{mm}^{-1}\right)\end{array}$ & 4.39 \\
\hline $\begin{array}{l}\text { Crystal dimensions } \\
(\mathrm{mm})\end{array}$ & $0.27 \times 0.16 \times 0.03$ & Absorption correction & Numerical \\
\hline Crystal system & Monoclinic & $F(000)$ & 308 \\
\hline Space group & $P 2_{1}$ & $\theta_{\min }$ to $\theta_{\max }\left({ }^{\circ}\right)$ & 2.9 to 70.0 \\
\hline$Z$ & 2 & Measured reflections & 10189 \\
\hline$a(\AA)$ & $5.8040(2)$ & $\begin{array}{l}\text { Independent } \\
\text { reflections }\end{array}$ & $\begin{array}{l}2389 \\
\left(R_{\text {int }}=0.023\right)\end{array}$ \\
\hline$b(\AA)$ & $7.1933(2)$ & $\begin{array}{l}\text { Data/restraints/param } \\
\text { eters }\end{array}$ & $2389 / 1 / 155$ \\
\hline$c(\AA)$ & $15.2241(5)$ & Maximum shift/error & $<0.001$ \\
\hline$\alpha\left(^{\circ}\right)$ & 90.00 & Goodness-of-fit on $\mathrm{F}^{2}$ & 1.11 \\
\hline$\beta\left(^{\circ}\right)$ & $94.904(1)$ & $\begin{array}{l}\text { Final } R \text { indices }(I> \\
2 \sigma(I))\end{array}$ & $\begin{array}{l}R_{1}=0.021 \\
w R_{2}=0.052\end{array}$ \\
\hline
\end{tabular}




\begin{tabular}{llll}
$\gamma\left(^{\circ}\right)$ & 90.00 & $\mathrm{R}$ indices (all data) & $\begin{array}{l}R_{1}=0.021 \\
w R_{2}=0.052\end{array}$ \\
Collection ranges & $\begin{array}{l}h=-7 \rightarrow 7 \\
k=-8 \rightarrow 8\end{array}$ & $\begin{array}{l}\text { absolute structure } \\
\text { parameter }\end{array}$ & $0.032(7)$ \\
& $\begin{array}{l}l=-18 \rightarrow 18 \\
100\end{array}$ & $\begin{array}{l}\text { Extinction coefficient } \\
\text { Largest diff. peak and } \\
\text { hole }\left(\mathrm{e} \AA^{-3}\right)\end{array}$ & $\begin{array}{l}\mathrm{N} / \mathrm{A} \\
0.36 \text { and }-0.57\end{array}$ \\
Temperature $(\mathrm{K})$ & $633.28(3)$ & & \\
\hline
\end{tabular}

\section{Crystallographic Data for derivative of 23 for stereochemical determination:}

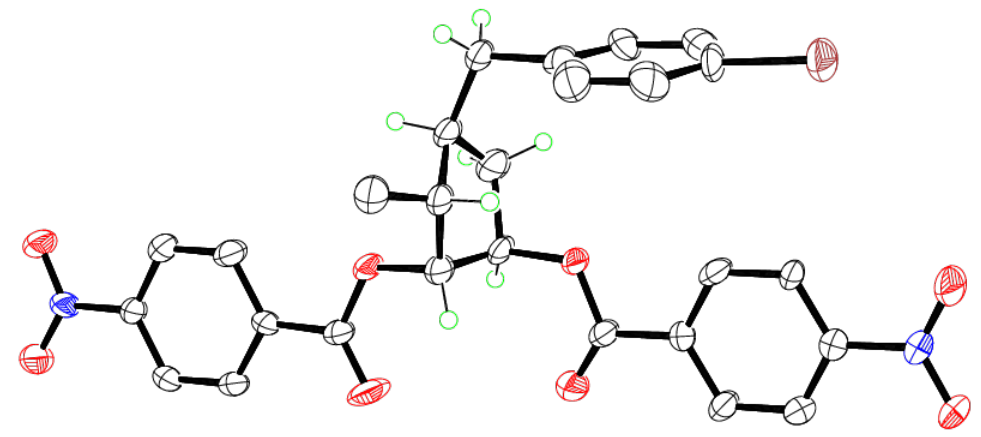

ORTEP representation of the solid state molecular structure of $\mathbf{2 3}$ derivative for stereochemical determination; ellipsoids drawn at 50\% probability, the majority of hydrogen atoms are omitted for clarity. Disordered solvent that could not be modeled has been addressed using a solvent mask with Olex2. The asymmetric unit contains a solvent molecule (chloroform) and two molecules of 23; only one molecule of $\mathbf{2 3}$ is shown for clarity.

\begin{tabular}{|c|c|c|c|}
\hline Empirical formula & $\begin{array}{l}\mathrm{C}_{27} \mathrm{H}_{23} \mathrm{BrN}_{2} \mathrm{O}_{8} \cdot 0.5 \\
\left(\mathrm{CHCl}_{3}\right)\end{array}$ & $\mathrm{D}_{\text {calcd }}\left(\mathrm{Mg} \mathrm{m}^{-3}\right)$ & 1.391 \\
\hline$F_{\mathrm{w}}$ & 643.07 & Radiation & $\begin{array}{l}\mathrm{Cu}_{\mathrm{K}_{a}}, \lambda= \\
1.54178 \AA\end{array}$ \\
\hline Colour, habit & Colourless, block & $\begin{array}{l}\text { Absorption coeff. }(\mu) \\
\left(\mathrm{mm}^{-1}\right)\end{array}$ & 3.44 \\
\hline $\begin{array}{l}\text { Crystal dimensions } \\
(\mathrm{mm})\end{array}$ & $0.41 \times 0.14 \times 0.10$ & Absorption correction & Numerical \\
\hline Crystal system & Triclinic & $F(000)$ & 654 \\
\hline Space group & $P 1$ & $\theta_{\min }$ to $\theta_{\max }\left(^{\circ}\right)$ & 4.4 to 66.7 \\
\hline$Z$ & 2 & Measured reflections & 24104 \\
\hline$a(\AA)$ & $9.4486(3)$ & $\begin{array}{l}\text { Independent } \\
\text { reflections }\end{array}$ & $\begin{array}{l}9926 \\
\left(R_{\text {int }}=0.046\right)\end{array}$ \\
\hline$b(\AA)$ & $11.0765(3)$ & $\begin{array}{l}\text { Data/restraints/param } \\
\text { eters }\end{array}$ & $9926 / 3 / 723$ \\
\hline$c(\AA)$ & $16.0570(5)$ & Maximum shift/error & $<0.001$ \\
\hline
\end{tabular}




\begin{tabular}{|c|c|c|c|}
\hline$\alpha\left(^{\circ}\right)$ & $73.497(2)$ & Goodness-of-fit on $\mathrm{F}^{2}$ & 1.03 \\
\hline$\beta\left(^{\circ}\right)$ & $87.912(2)$ & $\begin{array}{l}\text { Final } R \text { indices }(I> \\
2 \sigma(I))\end{array}$ & $\begin{array}{l}R_{1}=0.046 \\
w R_{2}=0.116\end{array}$ \\
\hline$\gamma\left(\left(^{\circ}\right)\right.$ & $72.630(2)$ & $\mathrm{R}$ indices (all data) & $\begin{array}{l}R_{1}=0.055 \\
w R_{2}=0.122\end{array}$ \\
\hline Collection ranges & $\begin{array}{l}h=-11 \rightarrow 11 \\
k=-13 \rightarrow 13 \\
l=-19 \rightarrow 19\end{array}$ & $\begin{array}{l}\text { absolute structure } \\
\text { parameter }\end{array}$ & $0.034(10)$ \\
\hline Temperature $(\mathrm{K})$ & 100 & Extinction coefficient & N/A \\
\hline Volume $\left(\AA^{3}\right)$ & $1535.63(8)$ & $\begin{array}{l}\text { Largest diff. peak and } \\
\text { hole }\left(\mathrm{e} \AA^{-3}\right)\end{array}$ & 0.99 and -0.52 \\
\hline
\end{tabular}


${ }^{1}$ Sigma-Aldrich Technical Bulletin. AL-143 Mineral Adsorbents, Filter Agents and Drying

${ }^{2}$ G. R. Fulmer, A. J. M. Miller, N. H. Sherden, H. E. Gottlieb, A. Nudelman, B. M. Stoltz, J. E. Bercaw and K. I. Goldberg, Organometallics, 2010, 29, 2176-2179.

${ }^{3}$ T. Henk, A. Giannis and K. Sandhoff, Liebigs Ann. der Chemie, 1992, 1992, 167-168.

${ }^{4}$ a) Y. Bourdreux, A. Lemetais, D. Urban, and J.-M. Beau, Chem. Comm. 2011, 47, 2146-2148. b) M. P. McLaughlin, L. L. Adduci, J. J. Becker and M. R. Gagné, J. Am. Chem. Soc. 2013, 135, 1225-1227. 

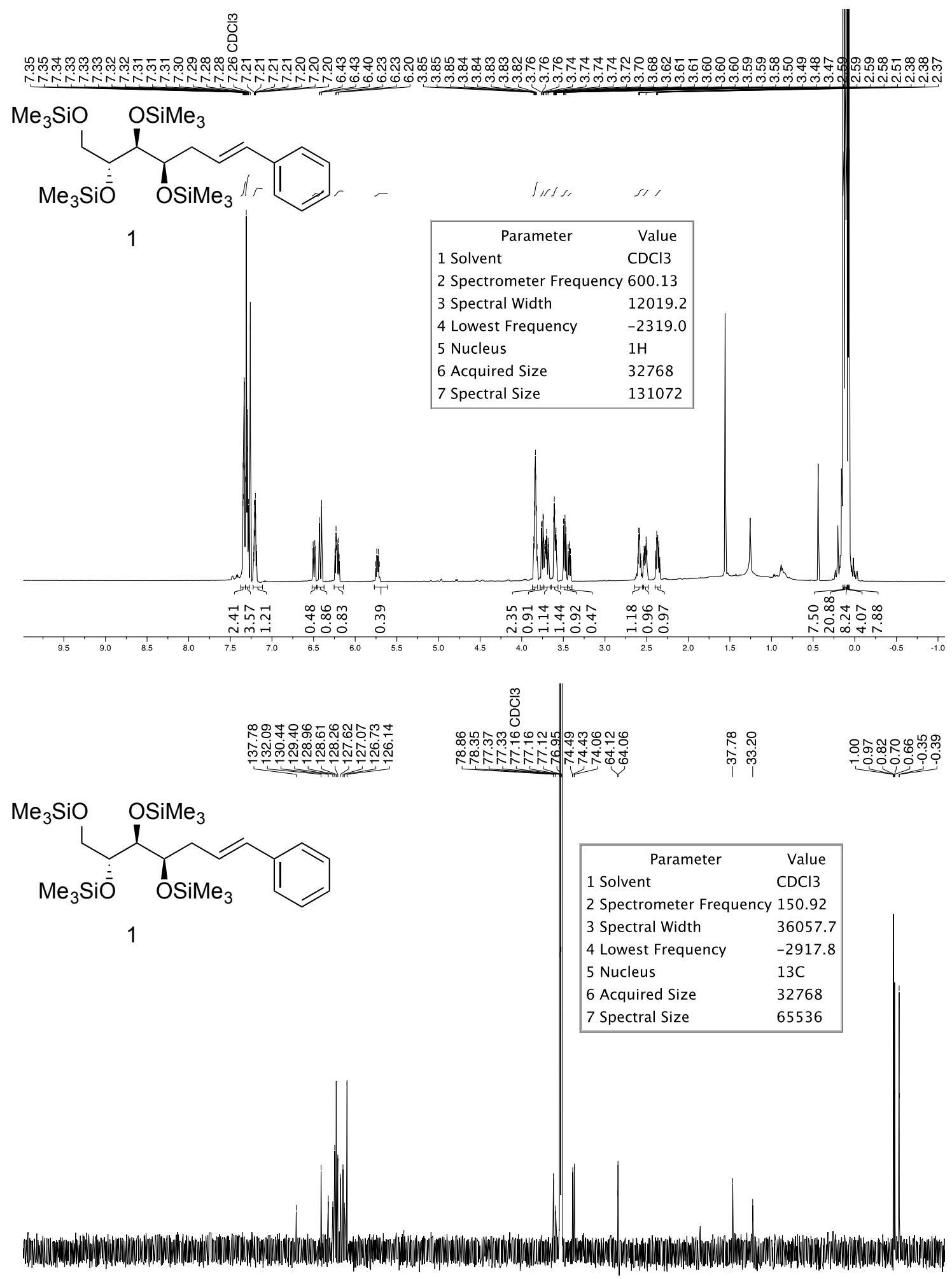

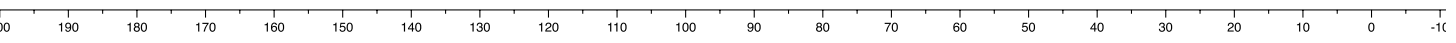




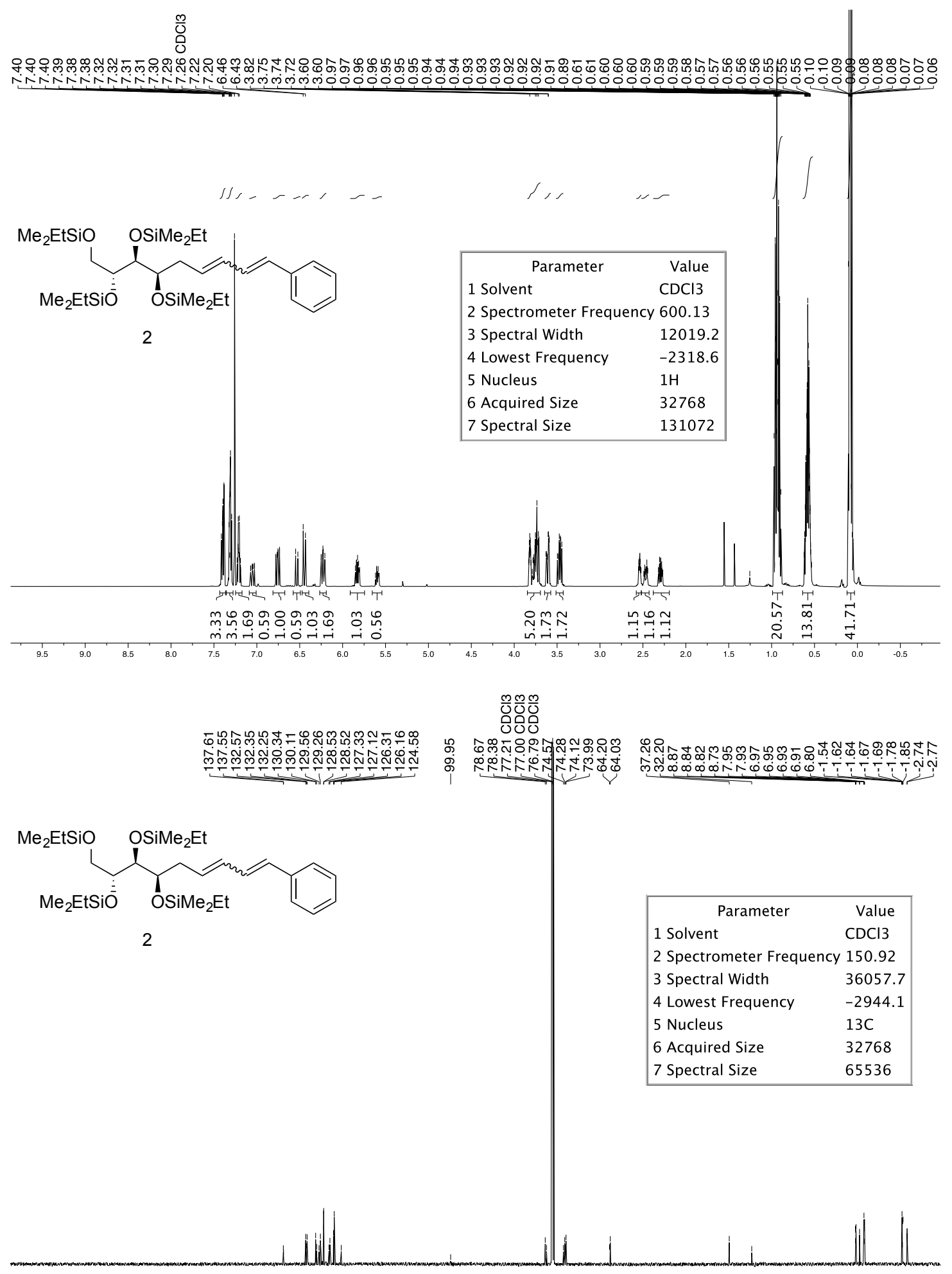

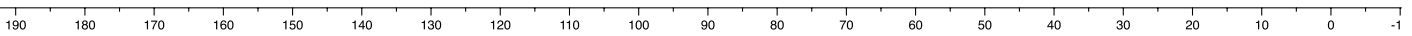



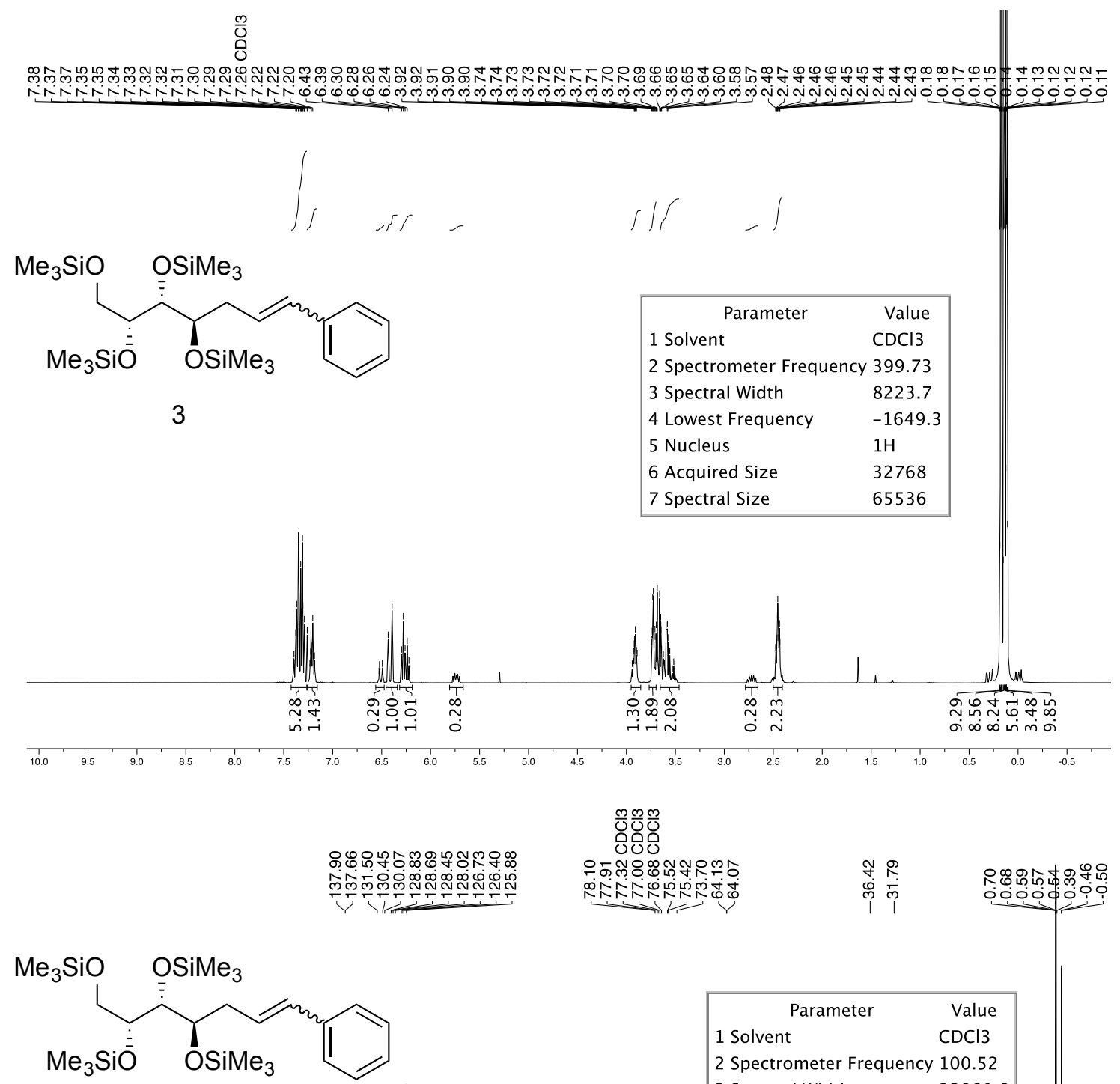

3

\begin{tabular}{|ll|}
\hline \multicolumn{1}{|c|}{ Parameter } & \multicolumn{1}{c|}{ Value } \\
1 Solvent & \multicolumn{1}{c|}{ CDCl3 } \\
2 Spectrometer Frequency 100.52 \\
3 Spectral Width & 23980.8 \\
4 Lowest Frequency & -1940.8 \\
5 Nucleus & $13 \mathrm{C}$ \\
6 Acquired Size & 32768 \\
7 Spectral Size & 65536 \\
\hline
\end{tabular}

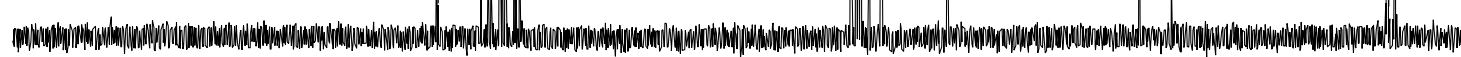




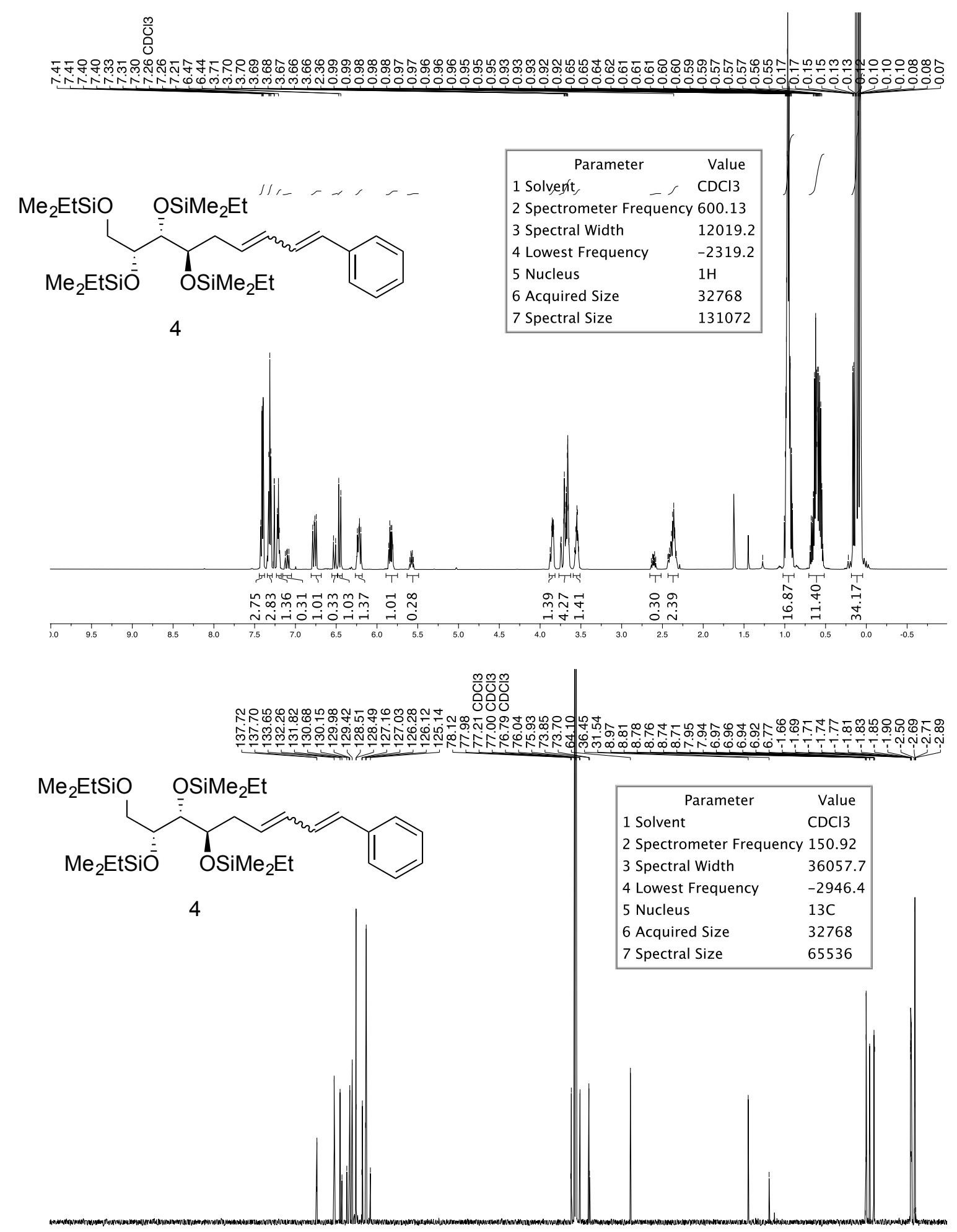

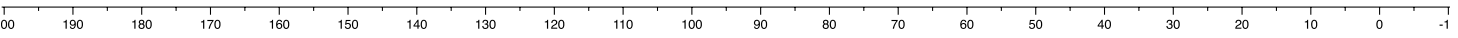



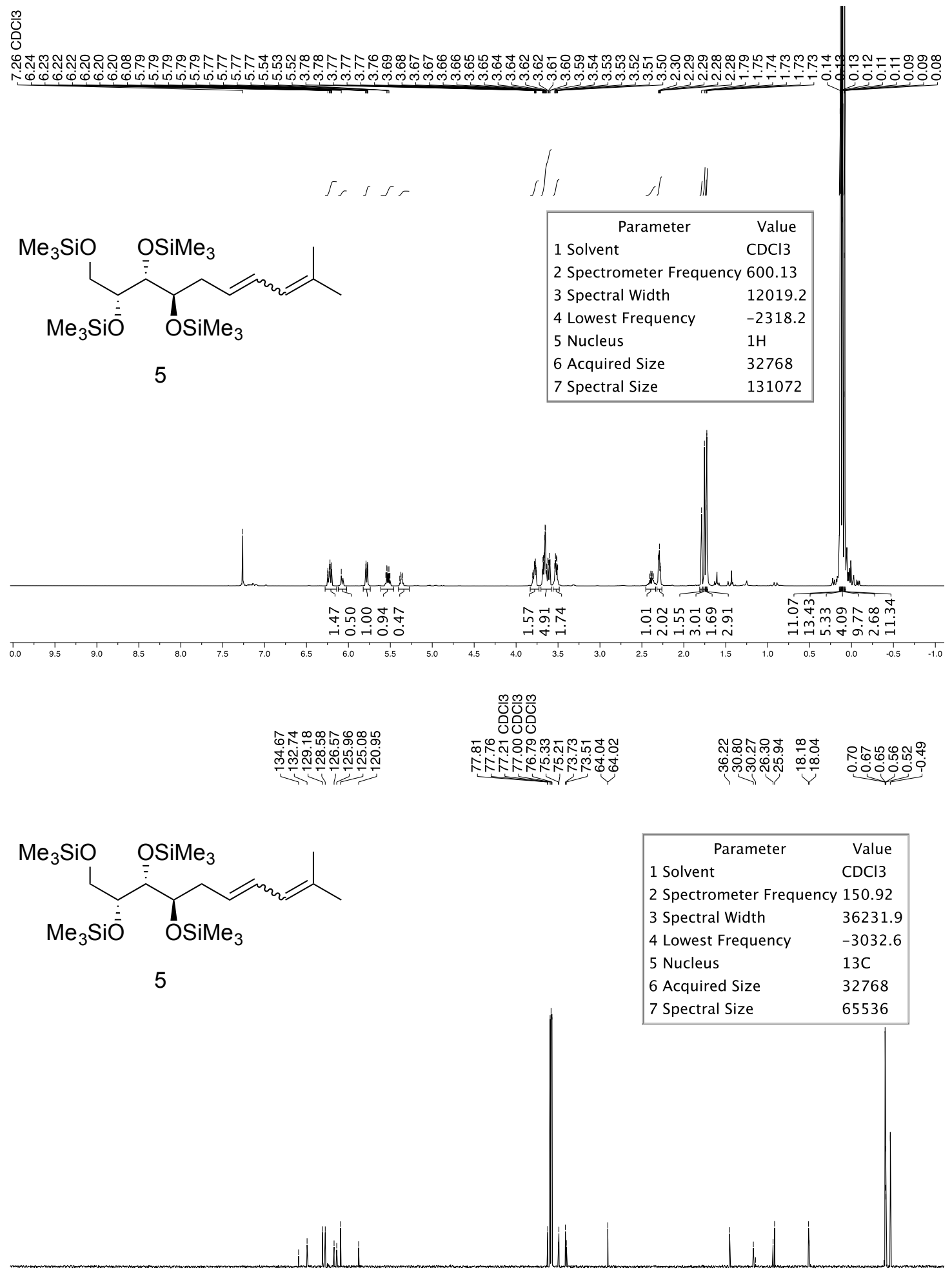

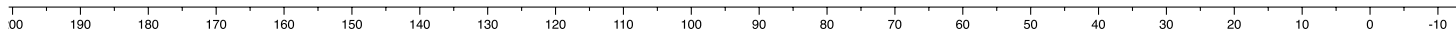




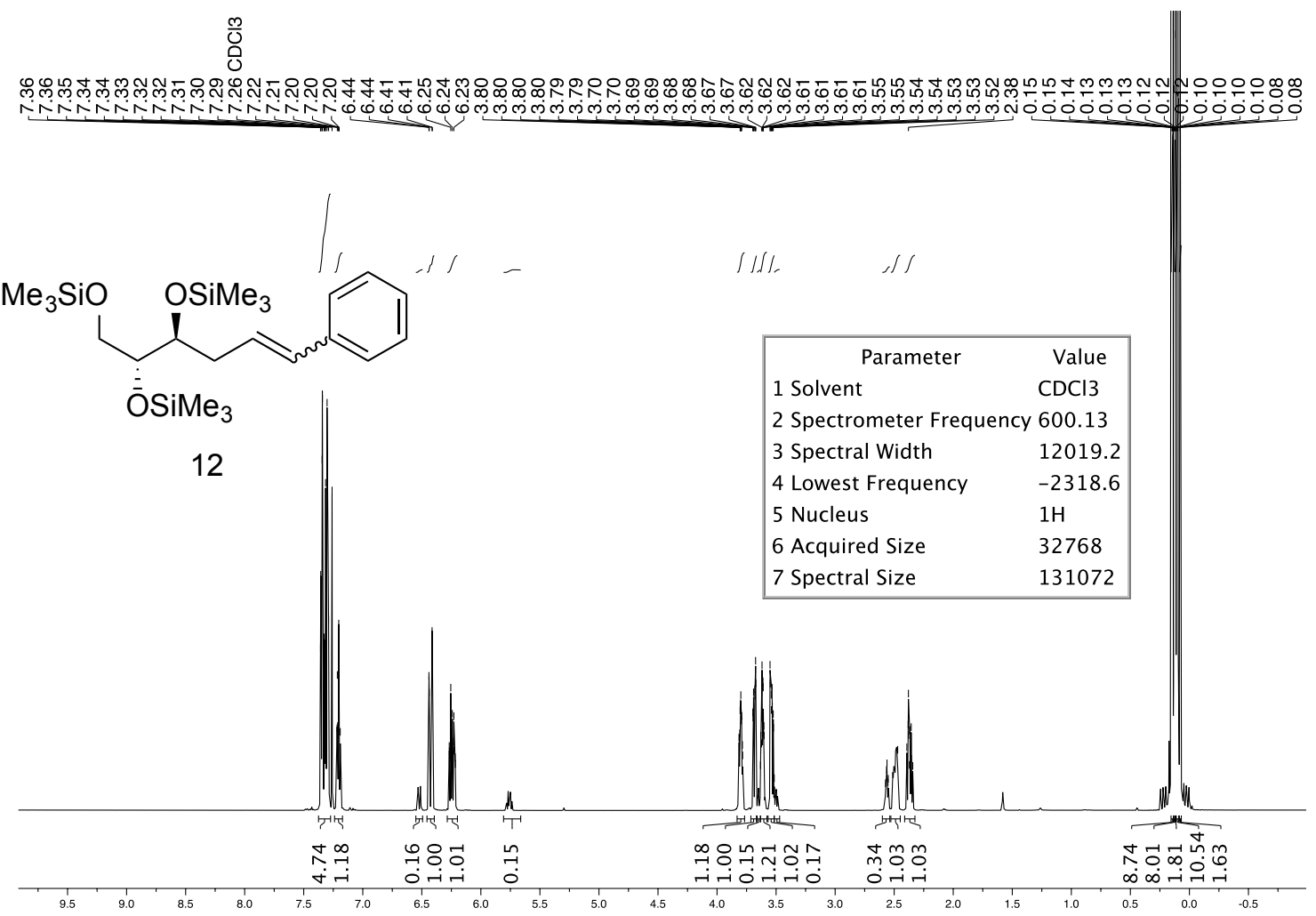

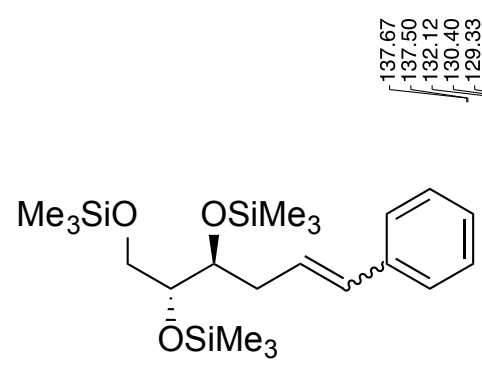

12

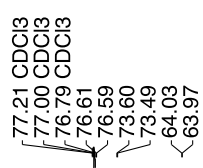

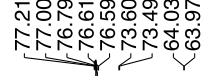

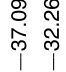

îj

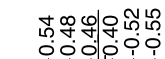

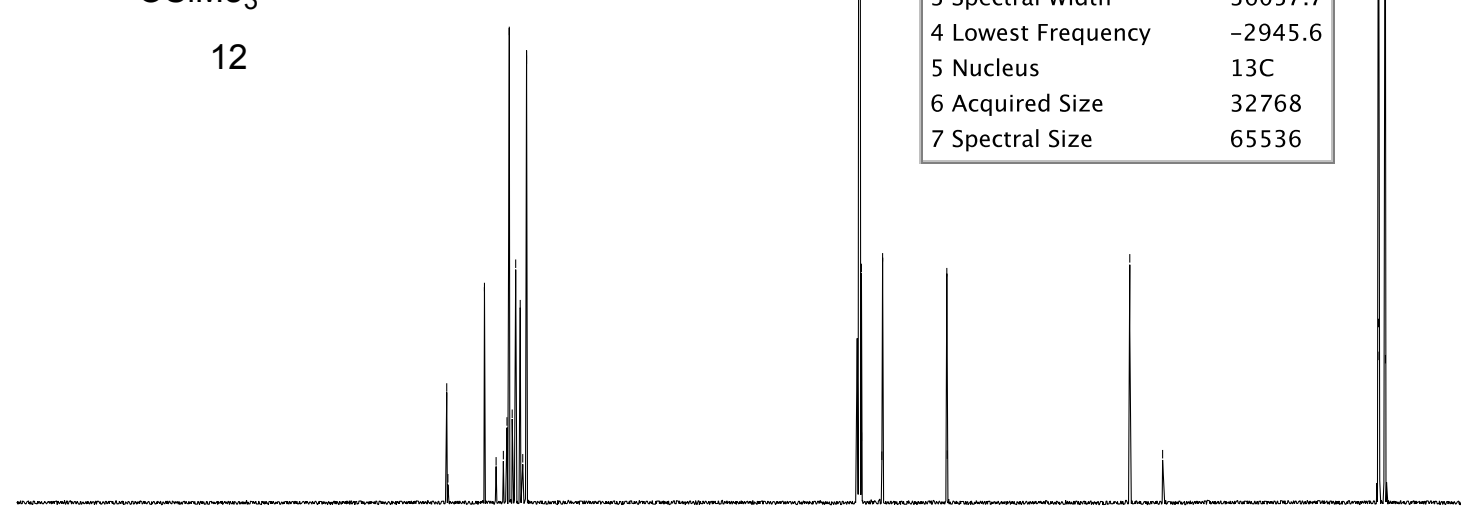

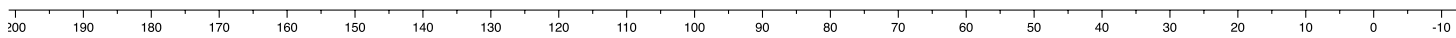



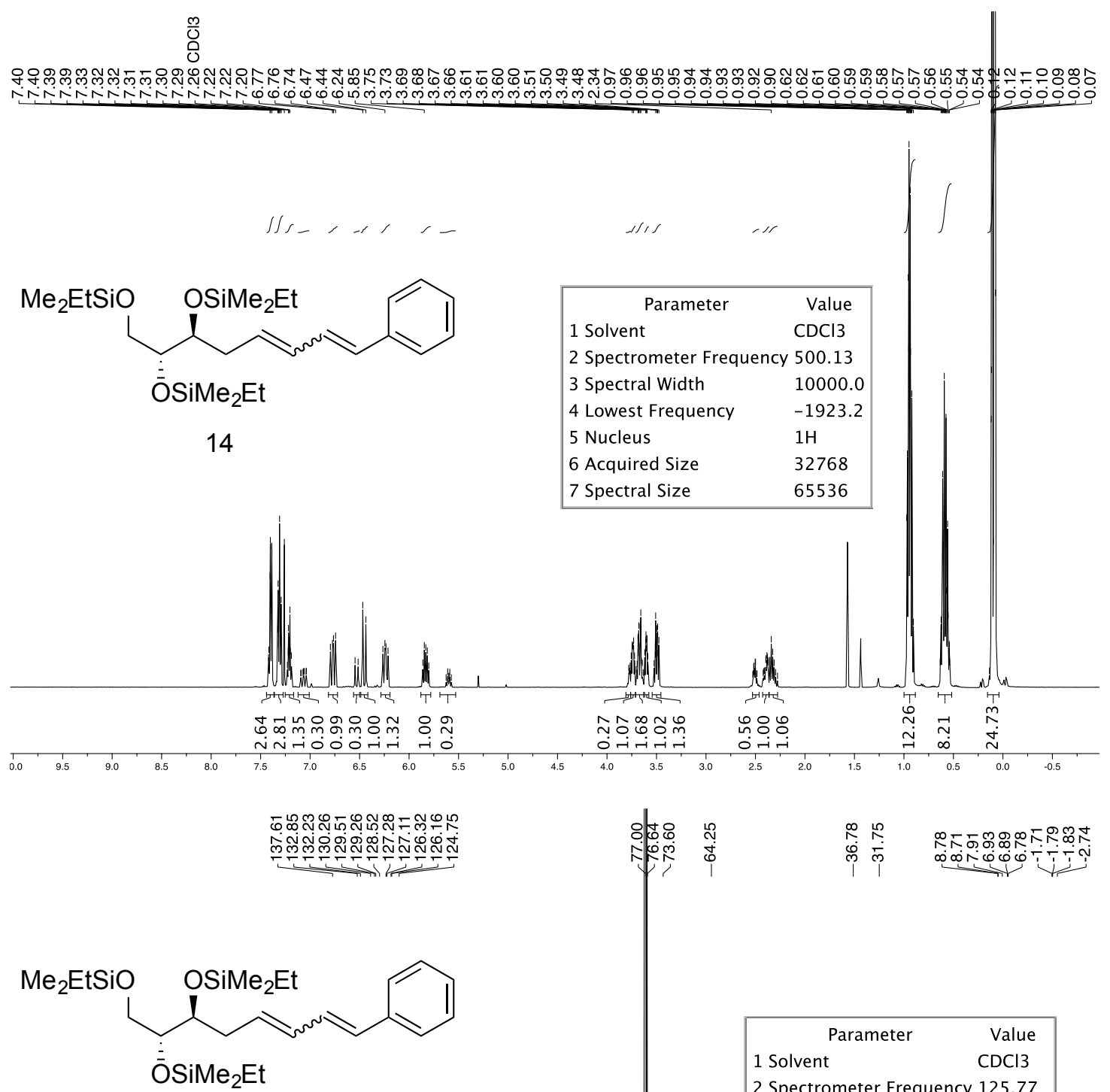

14

\begin{tabular}{|ll|}
\hline \multicolumn{1}{|c}{ Parameter } & \multicolumn{1}{c|}{ Value } \\
1 Solvent & \multicolumn{1}{c|}{$\begin{array}{c}125.77 \\
\text { 2 Spectrometer Frequency }\end{array}$} \\
3 Spectral Width & 29761.9 \\
4 Lowest Frequency & -2309.3 \\
5 Nucleus & $13 \mathrm{C}$ \\
6 Acquired Size & 32768 \\
7 Spectral Size & 65536 \\
\hline
\end{tabular}

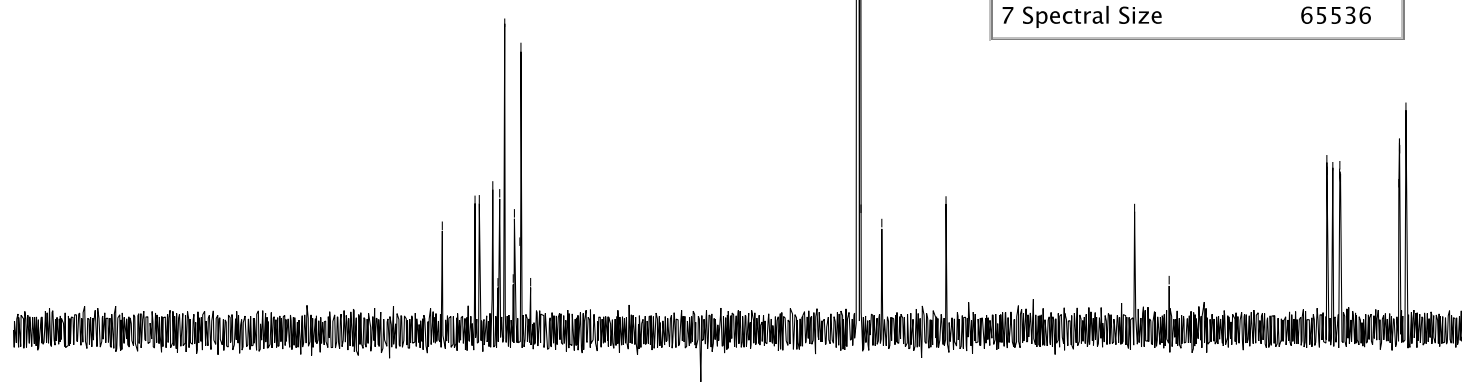

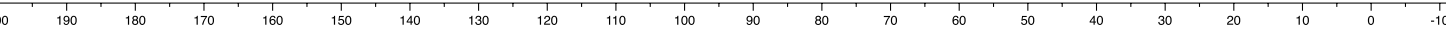




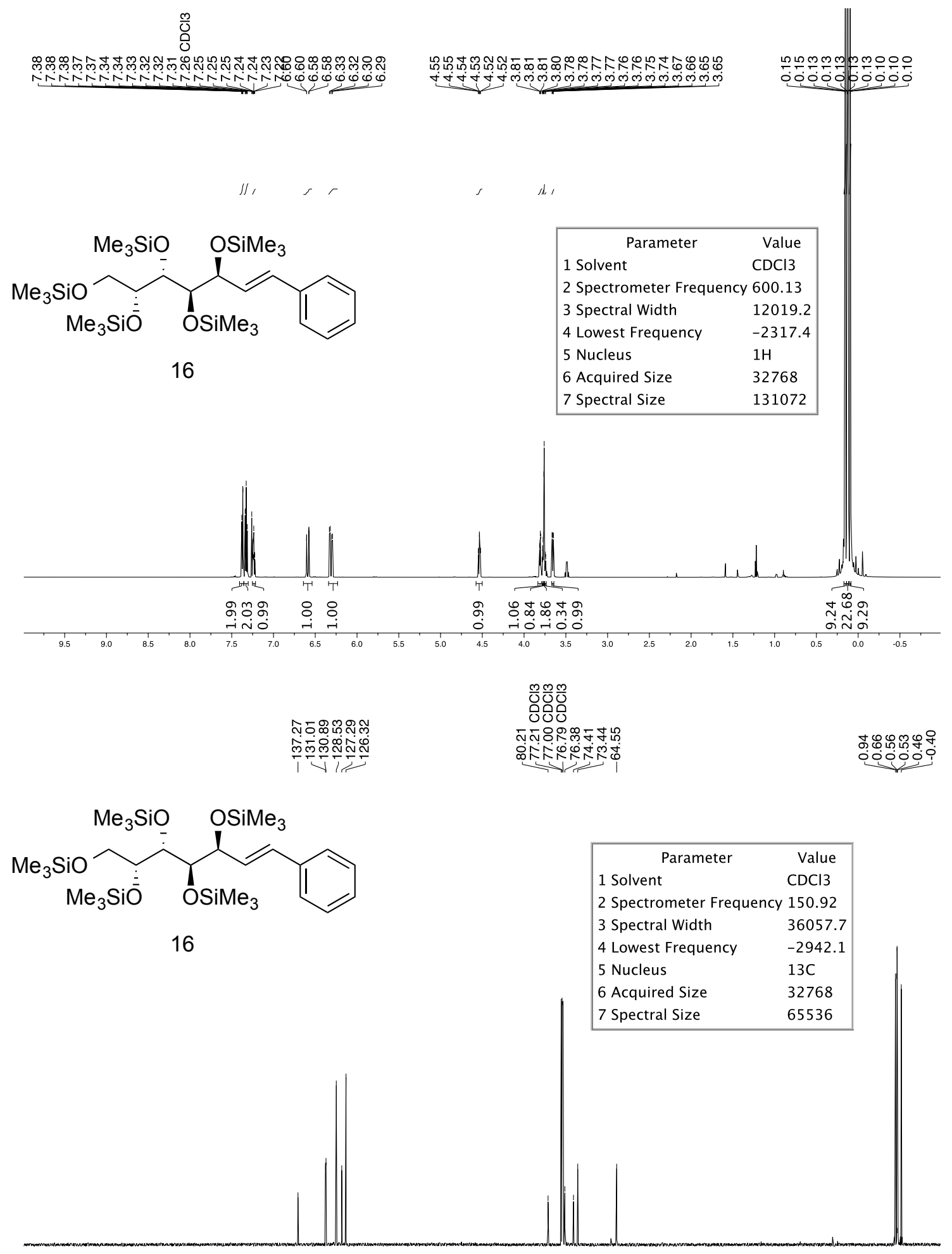

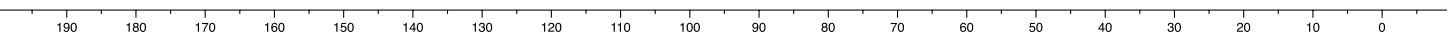




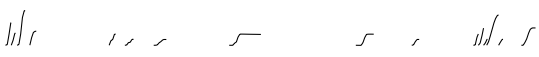

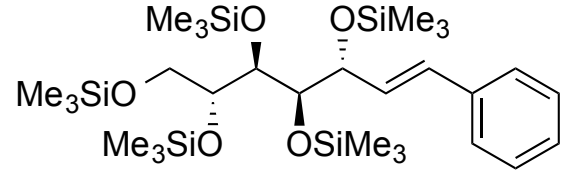

17

\begin{tabular}{|ll|}
\hline \multicolumn{1}{|c|}{ Parameter } & \multicolumn{1}{c|}{ Value } \\
1 Solvent & \multicolumn{1}{c|}{$\mathrm{CDCl}^{2}$} \\
2 Spectrometer Frequency & 600.13 \\
3 Spectral Width & 12019.2 \\
4 Lowest Frequency & -2318.6 \\
5 Nucleus & $1 \mathrm{H}$ \\
6 Acquired Size & 32768 \\
7 Spectral Size & 131072 \\
\hline
\end{tabular}

131072

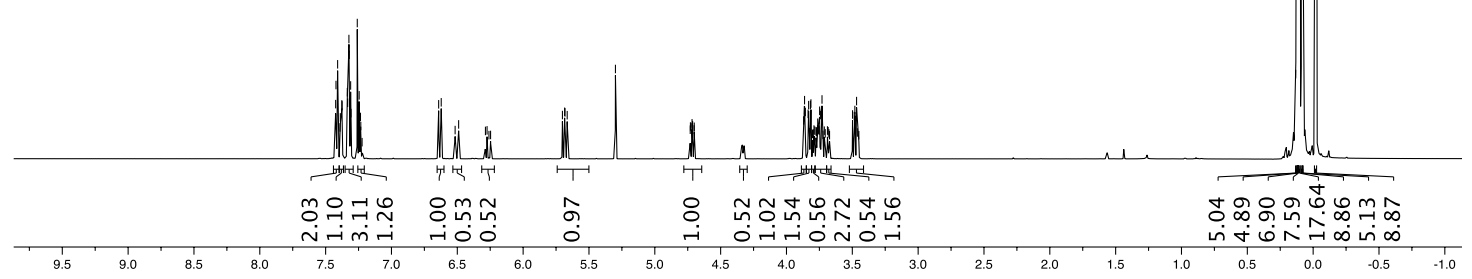

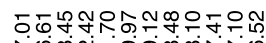

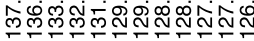

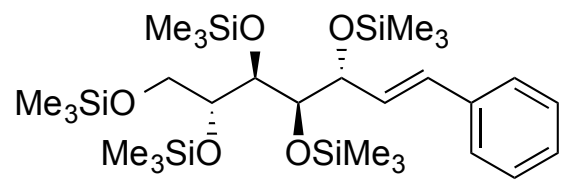

17

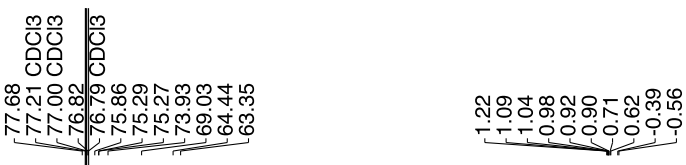

\begin{tabular}{|c|c|}
\hline Parameter & Value \\
\hline 1 Solvent & $\mathrm{CDCl} 3$ \\
\hline 2 Spectrometer Frequency & 150.92 \\
\hline 3 Spectral Width & 36057.7 \\
\hline 4 Lowest Frequency & -2941.6 \\
\hline 5 Nucleus & $13 \mathrm{C}$ \\
\hline 6 Acquired Size & 32768 \\
\hline 7 Spectral Size & 65536 \\
\hline
\end{tabular}

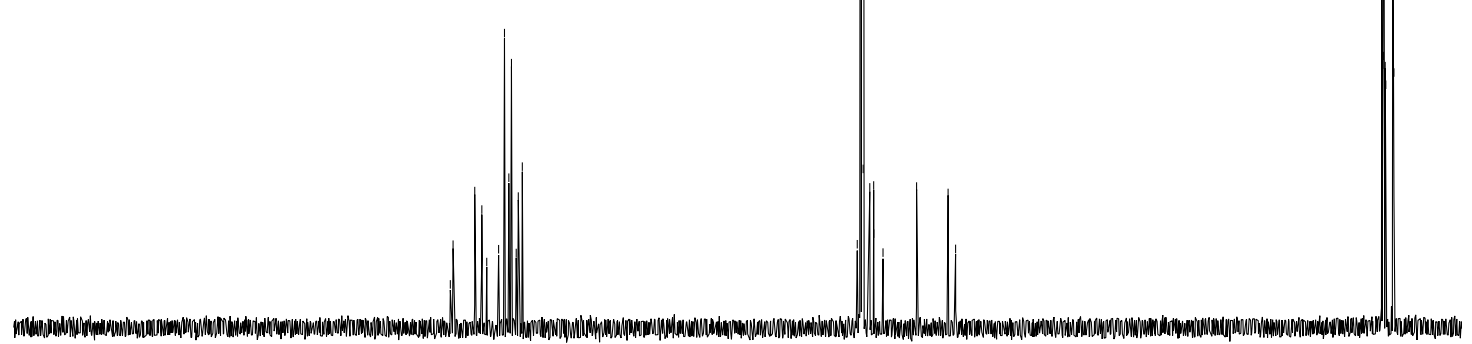

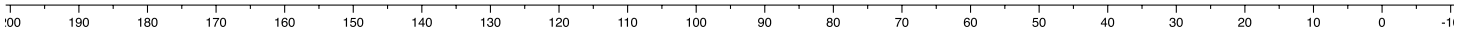




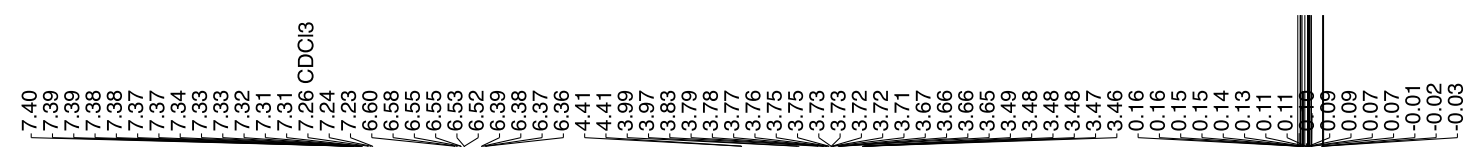

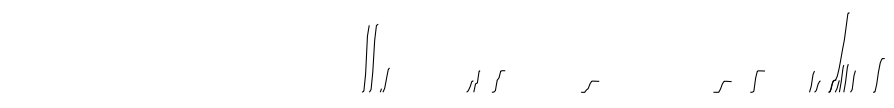

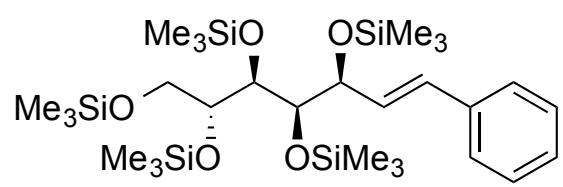

18

\begin{tabular}{|ll|}
\hline \multicolumn{1}{|c}{ Parameter } & \multicolumn{1}{c|}{ Value } \\
1 Solvent & \multicolumn{1}{c|}{ CDCl3 } \\
2 Spectrometer Frequency & 600.13 \\
3 Spectral Width & 12019.2 \\
4 Lowest Frequency & -2318.0 \\
5 Nucleus & $1 \mathrm{H}$ \\
6 Acquired Size & 32768 \\
7 Spectral Size & 131072 \\
\hline
\end{tabular}

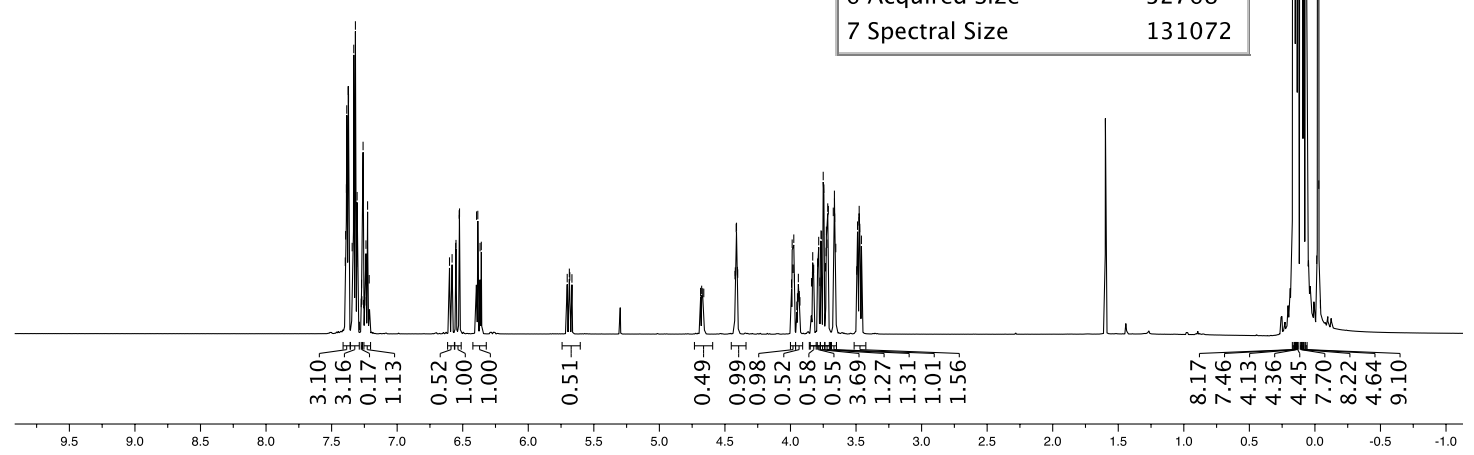

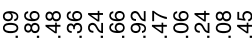

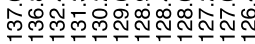

뜽 뜨음

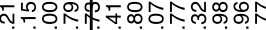

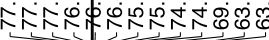

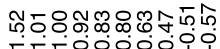
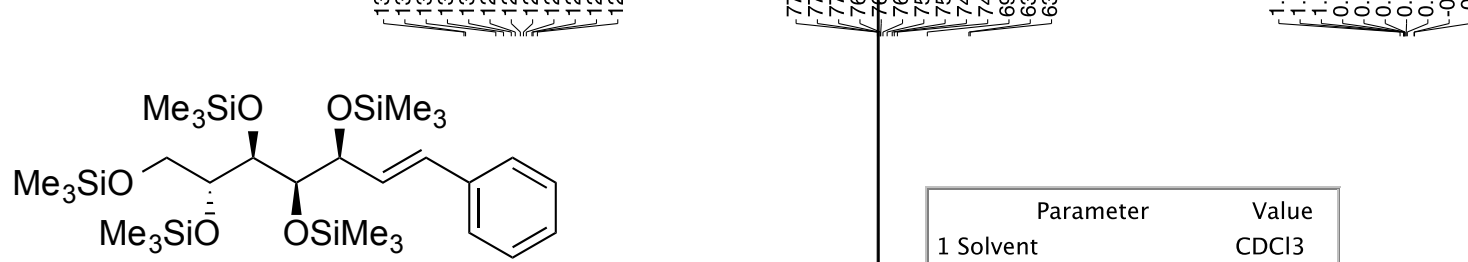

18

\begin{tabular}{|ll|}
\hline \multicolumn{1}{|c|}{ Parameter } & \multicolumn{1}{c|}{ Value } \\
1 Solvent & \multicolumn{1}{c|}{$\mathrm{CDCl} 3$} \\
2 Spectrometer Frequency & 150.92 \\
3 Spectral Width & 36057.7 \\
4 Lowest Frequency & -2941.3 \\
5 Nucleus & $13 \mathrm{C}$ \\
6 Acquired Size & 32768 \\
7 Spectral Size & 65536 \\
\hline
\end{tabular}

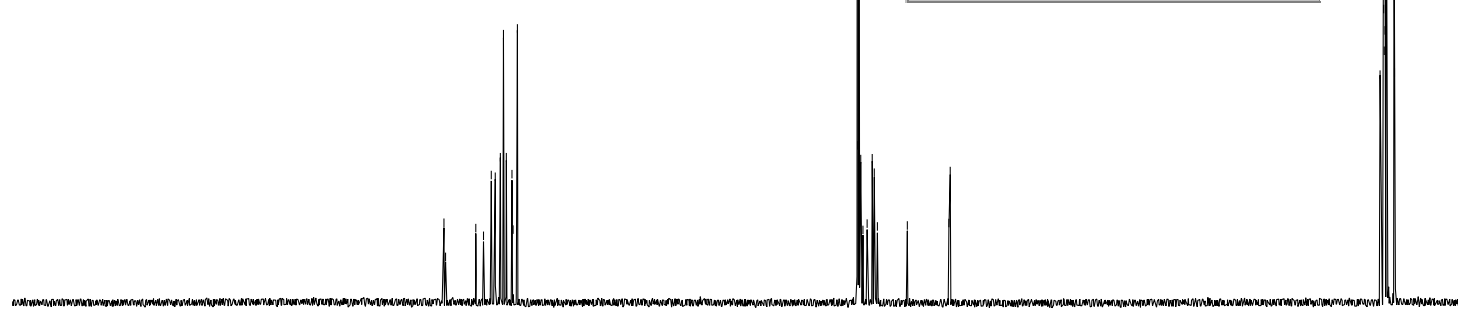



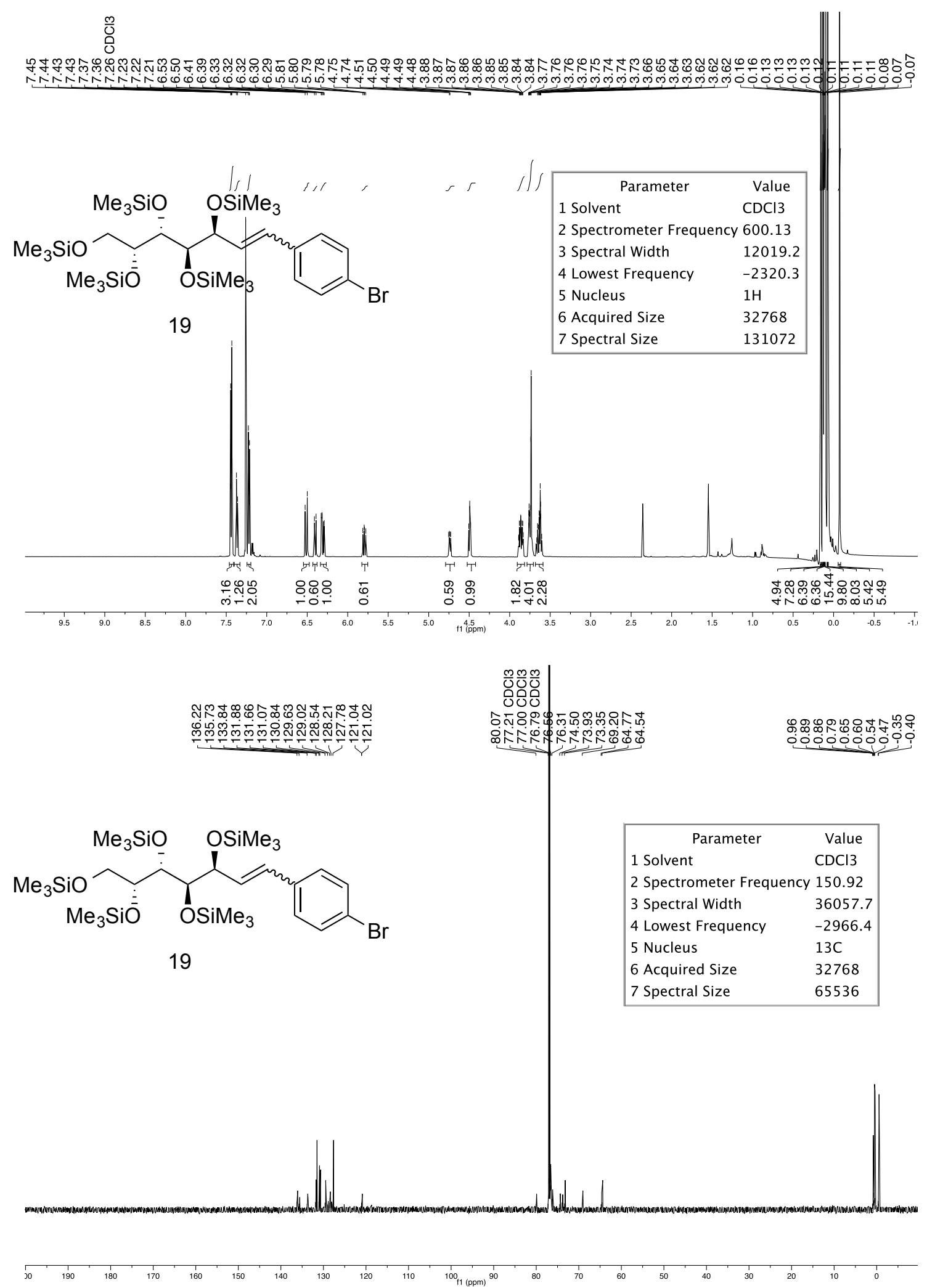


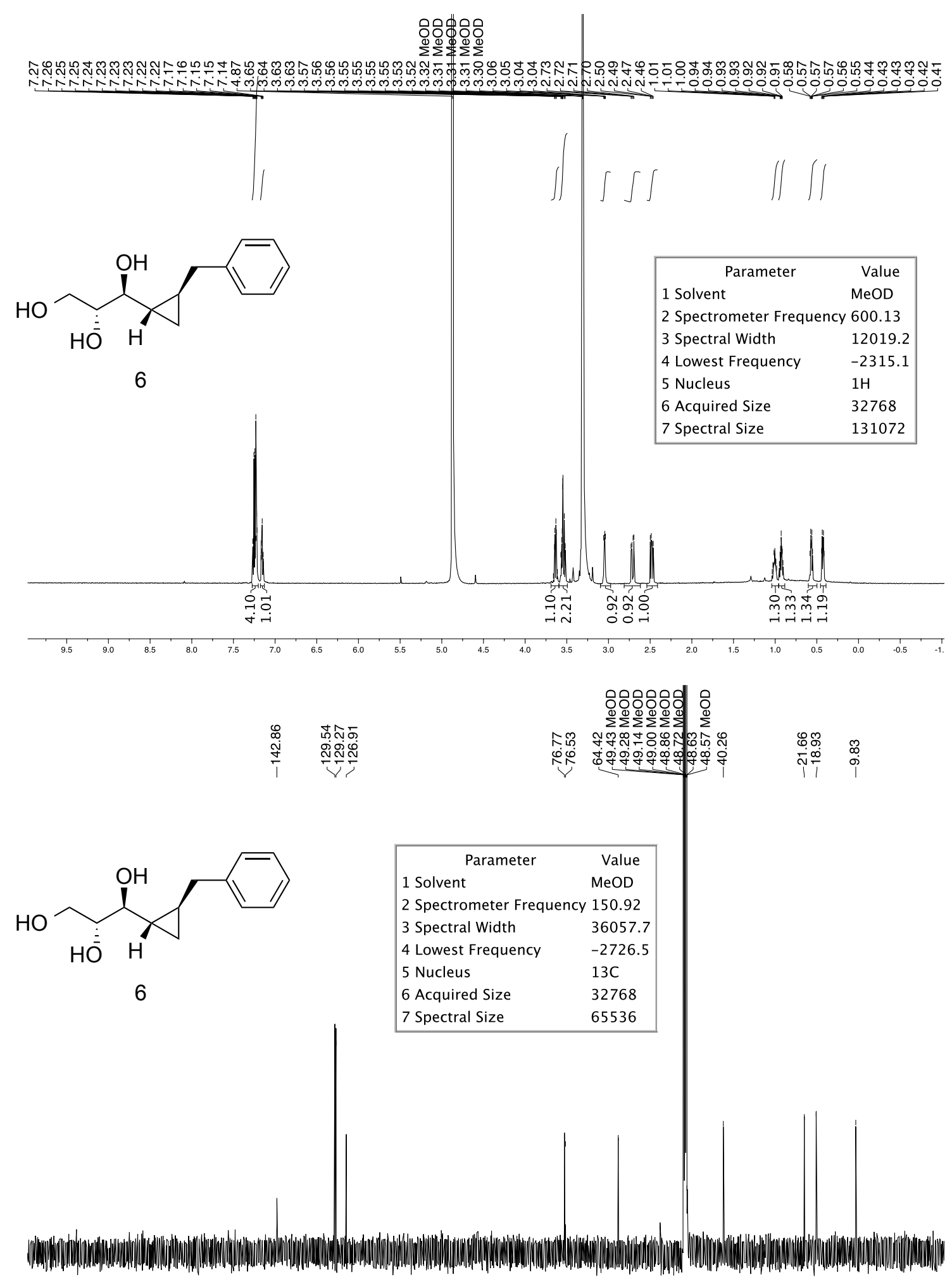

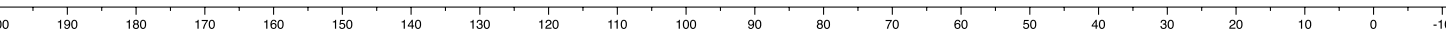



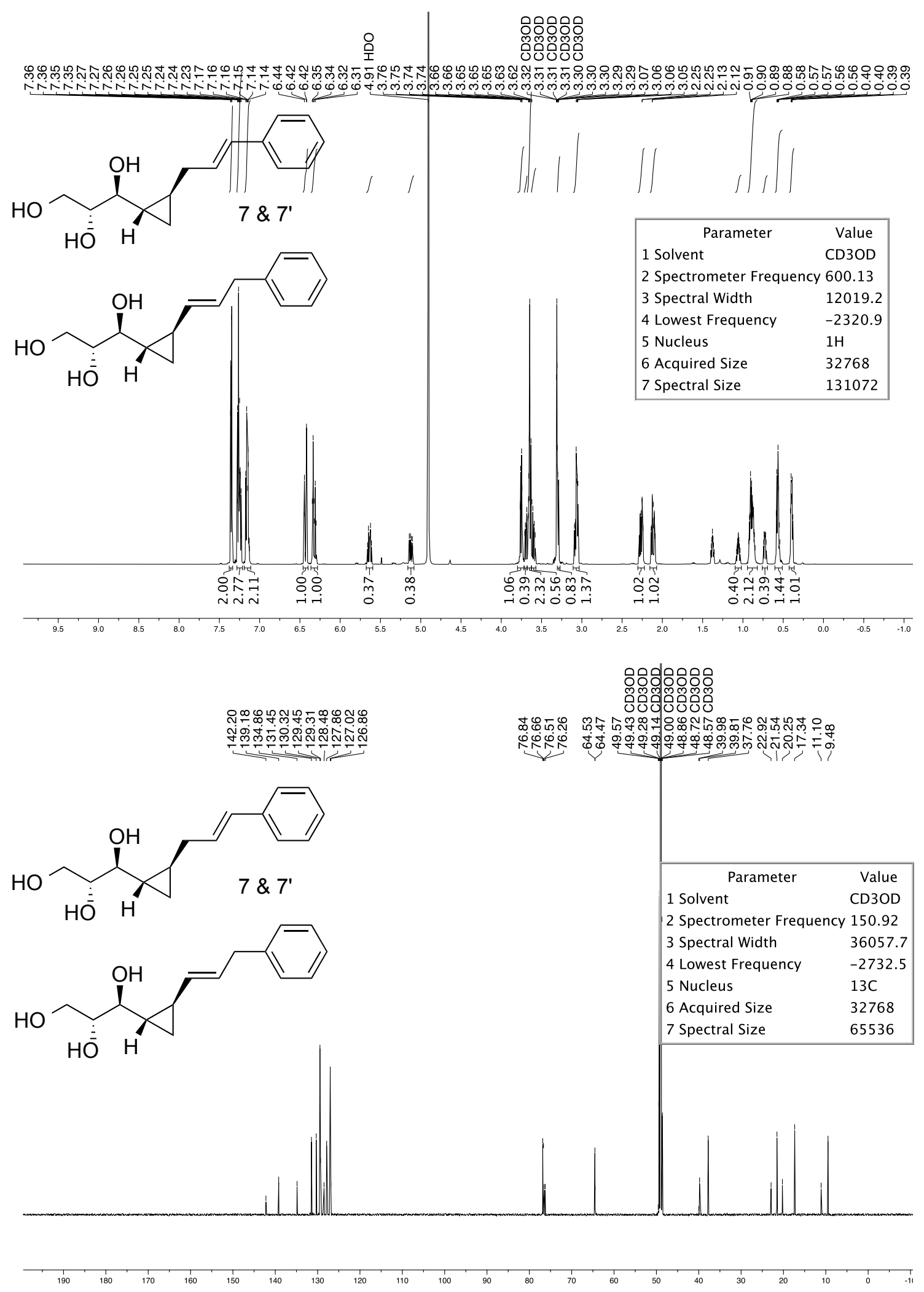


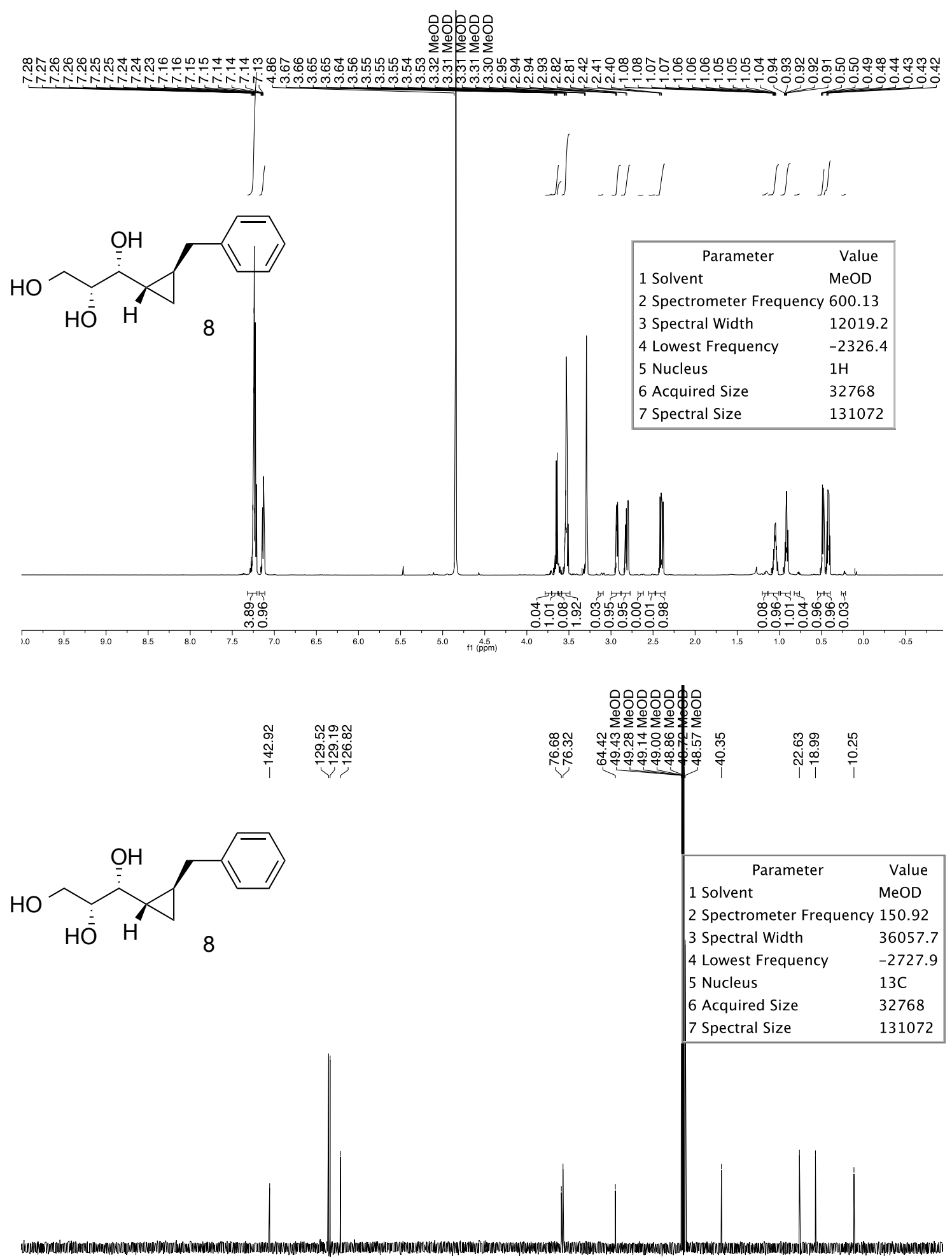

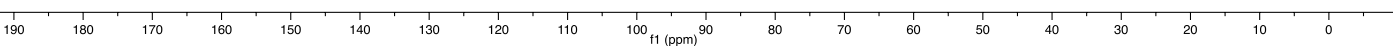




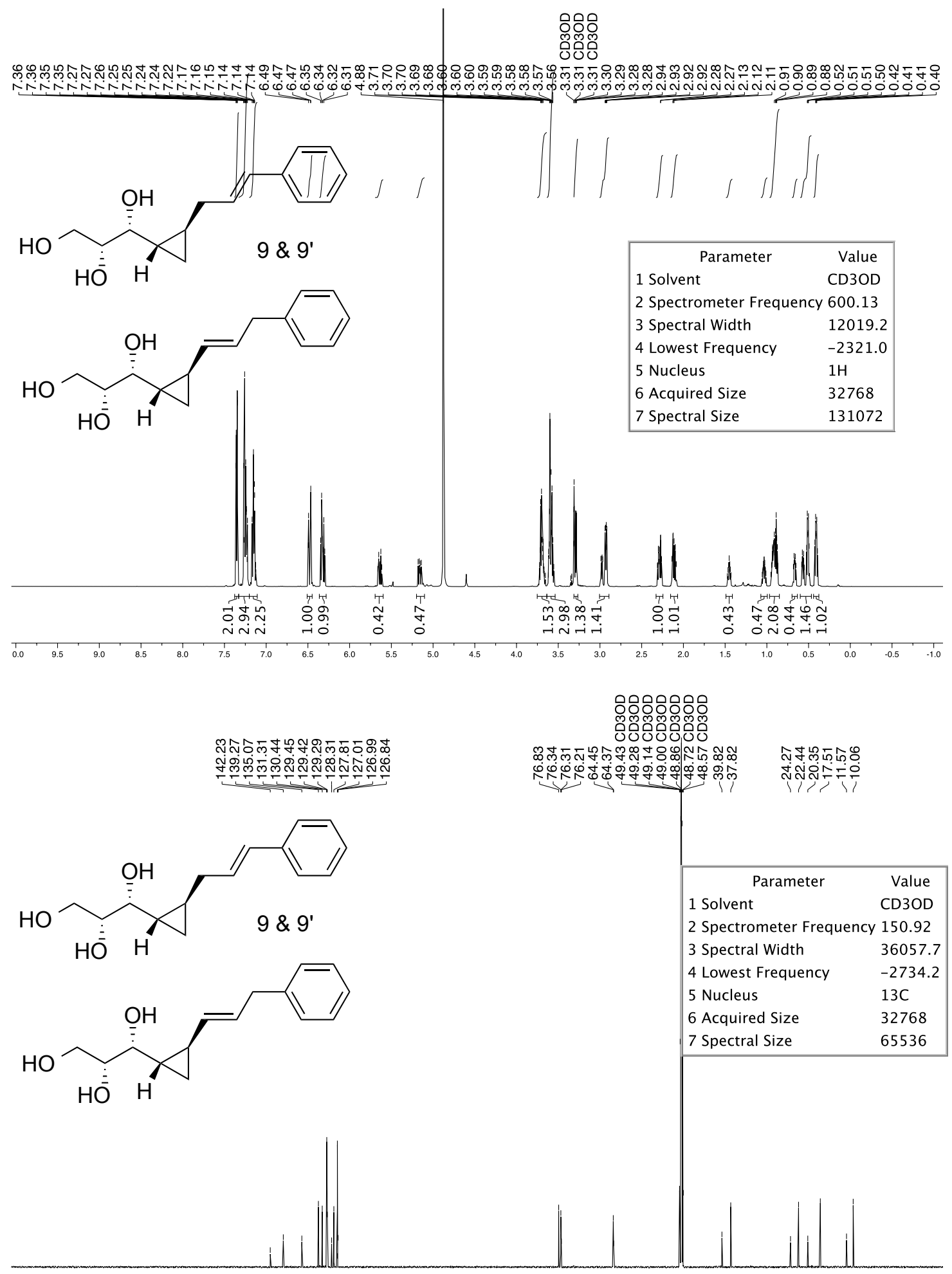

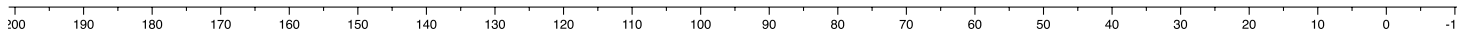



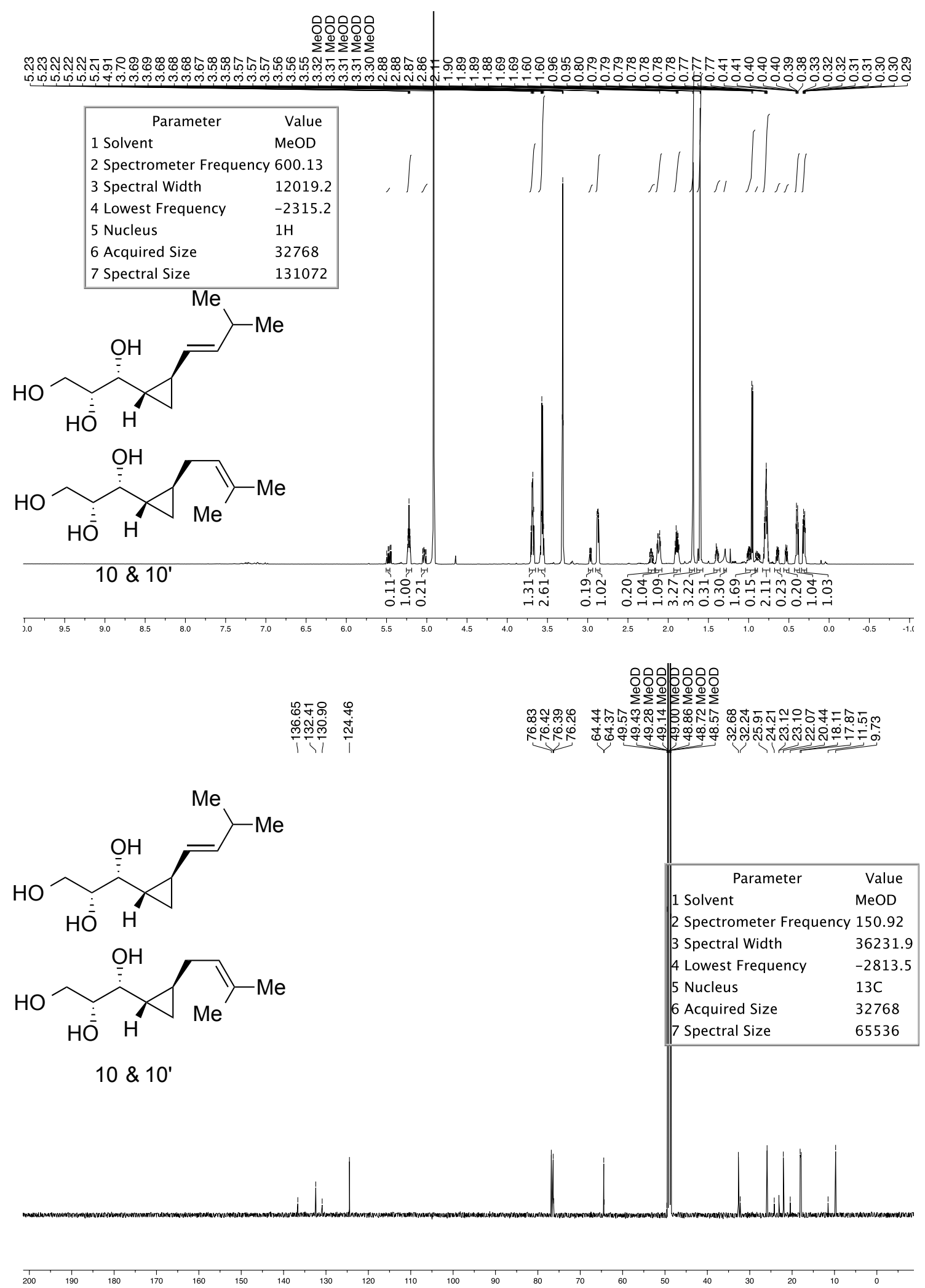


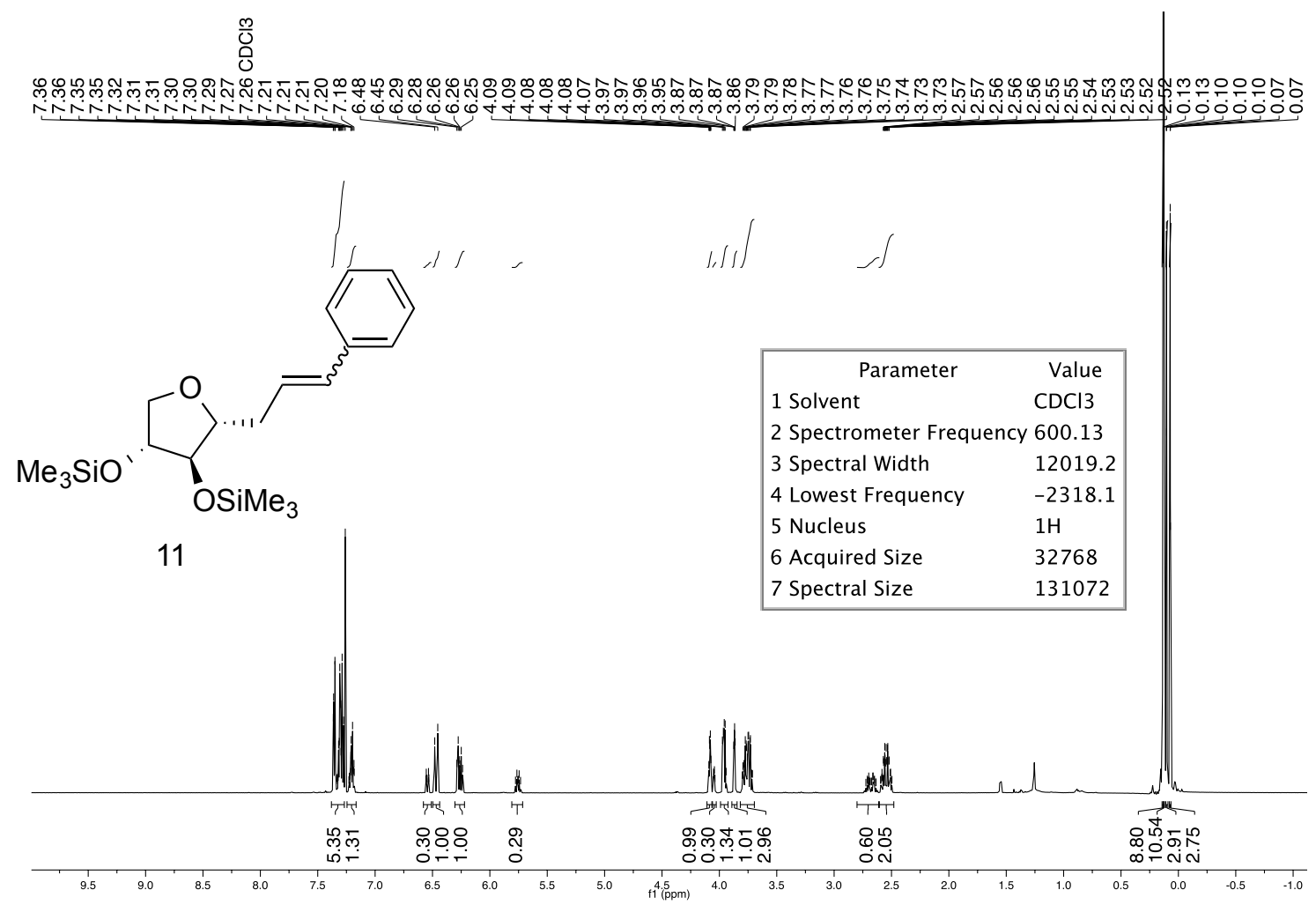

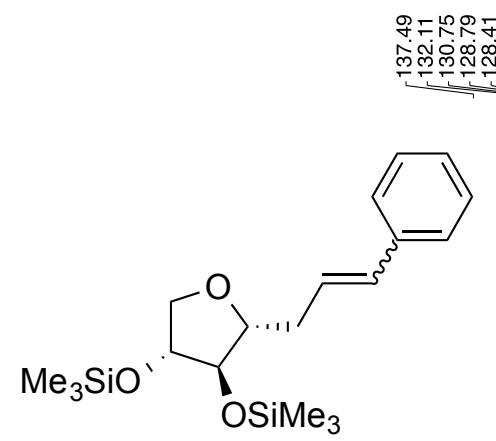

11
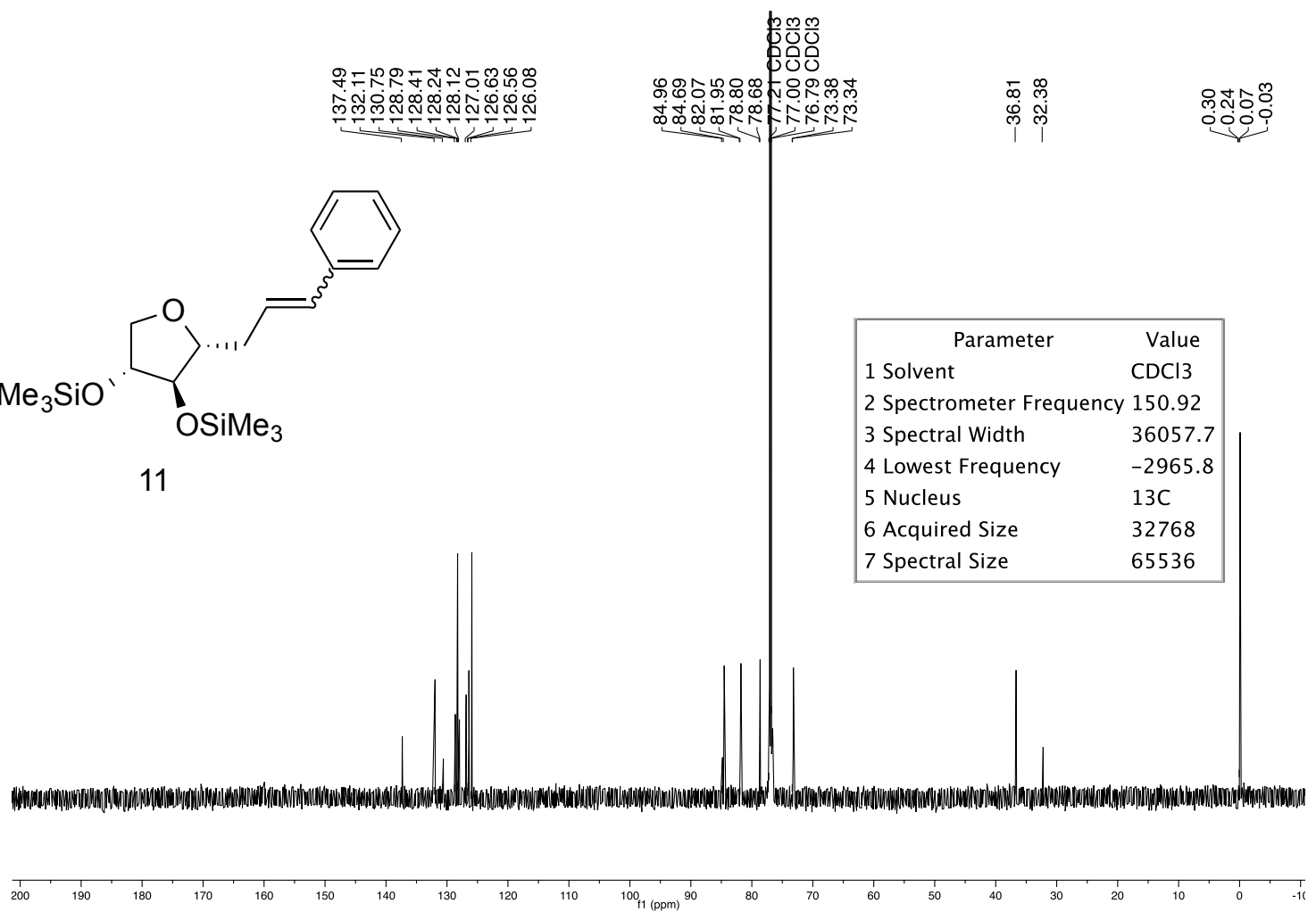


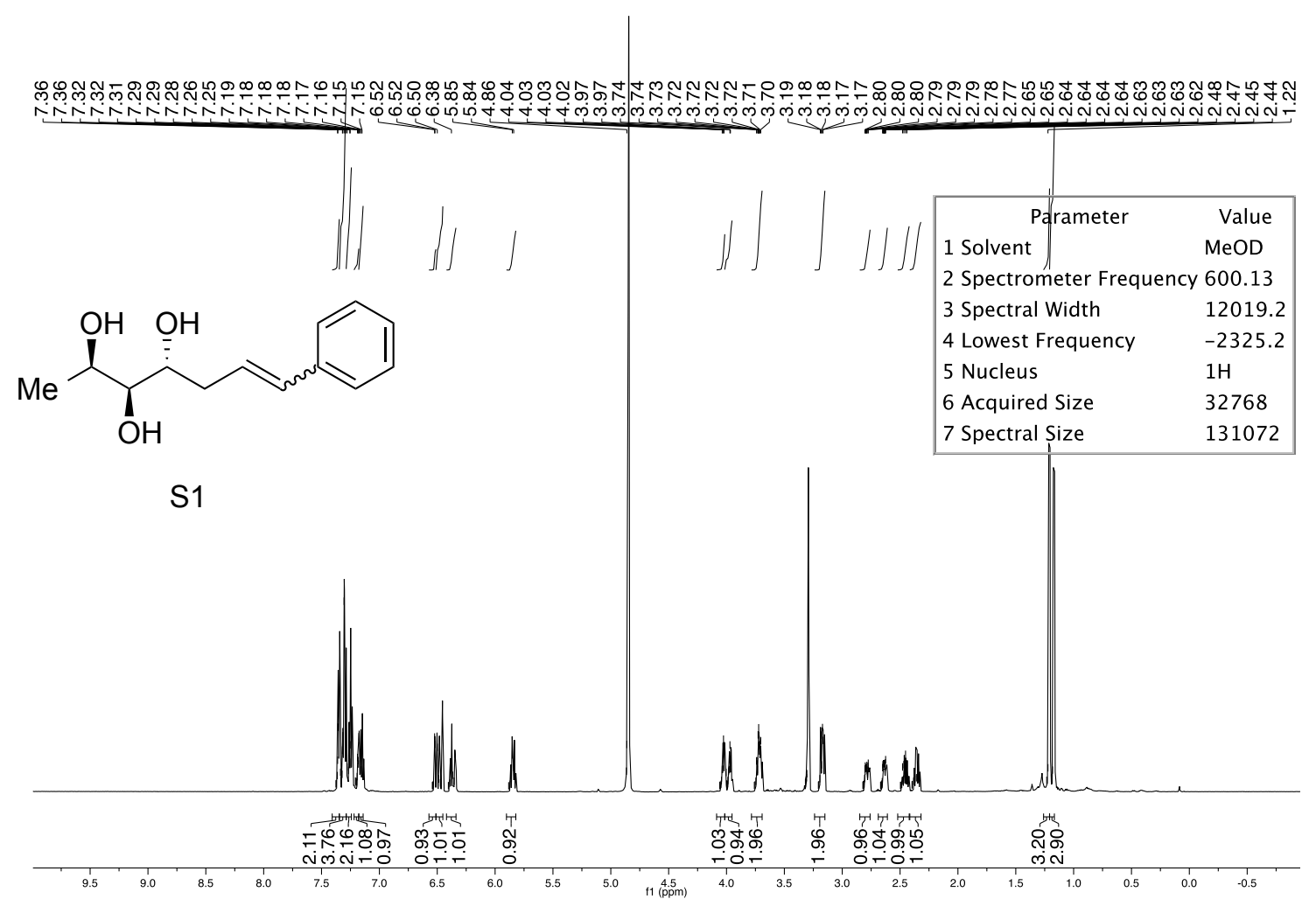

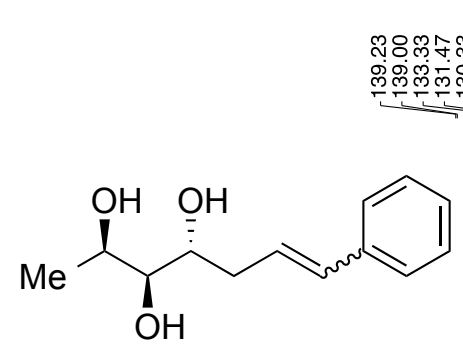

S1

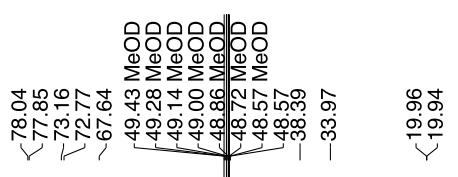

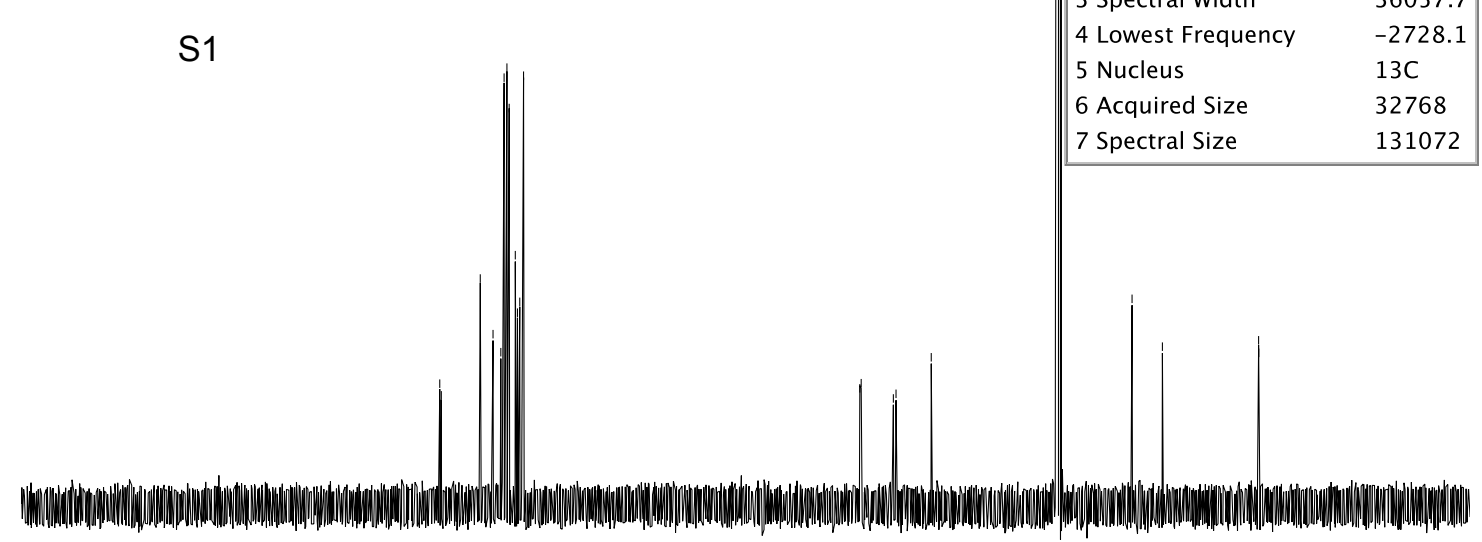

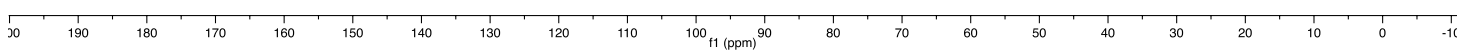



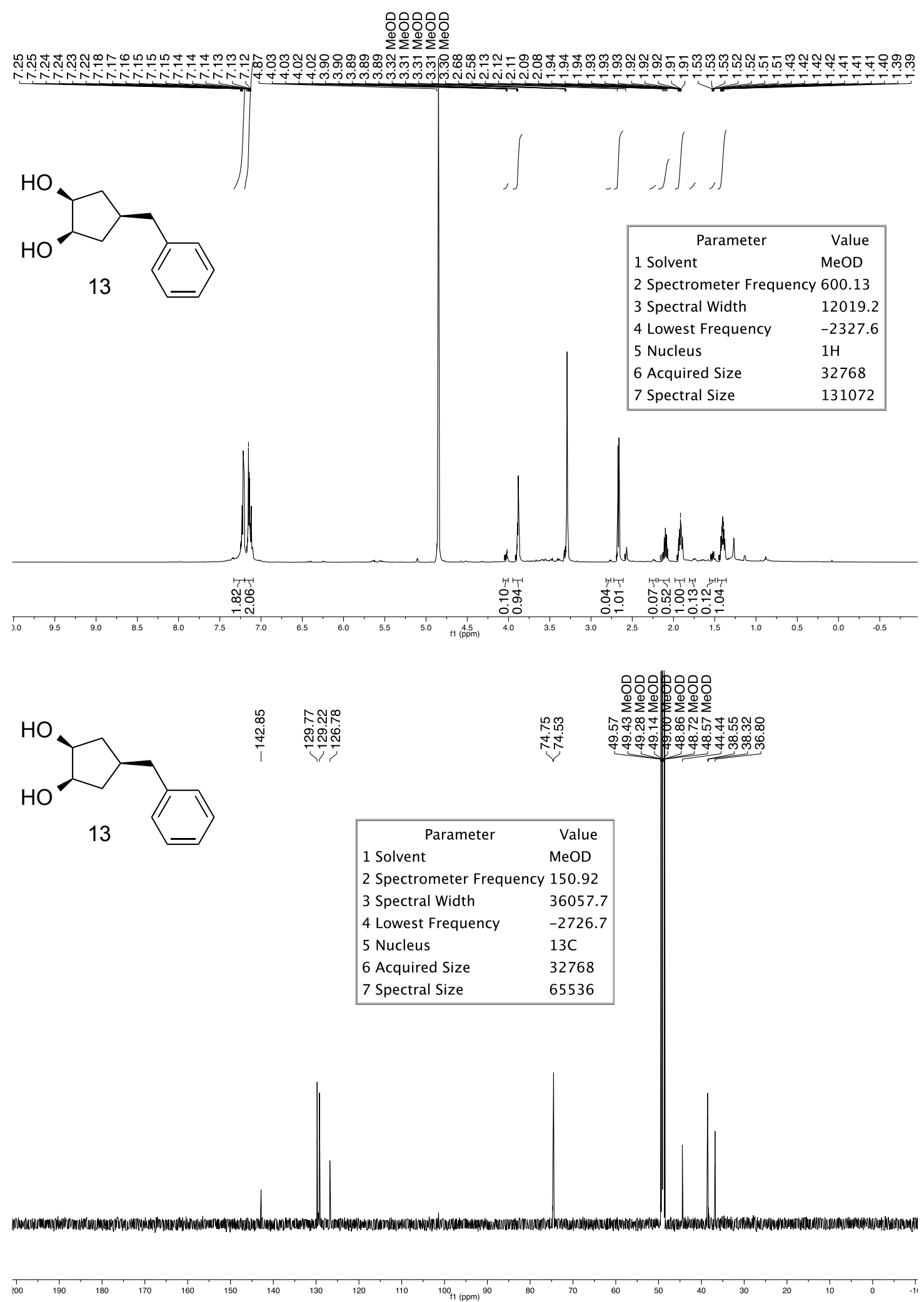

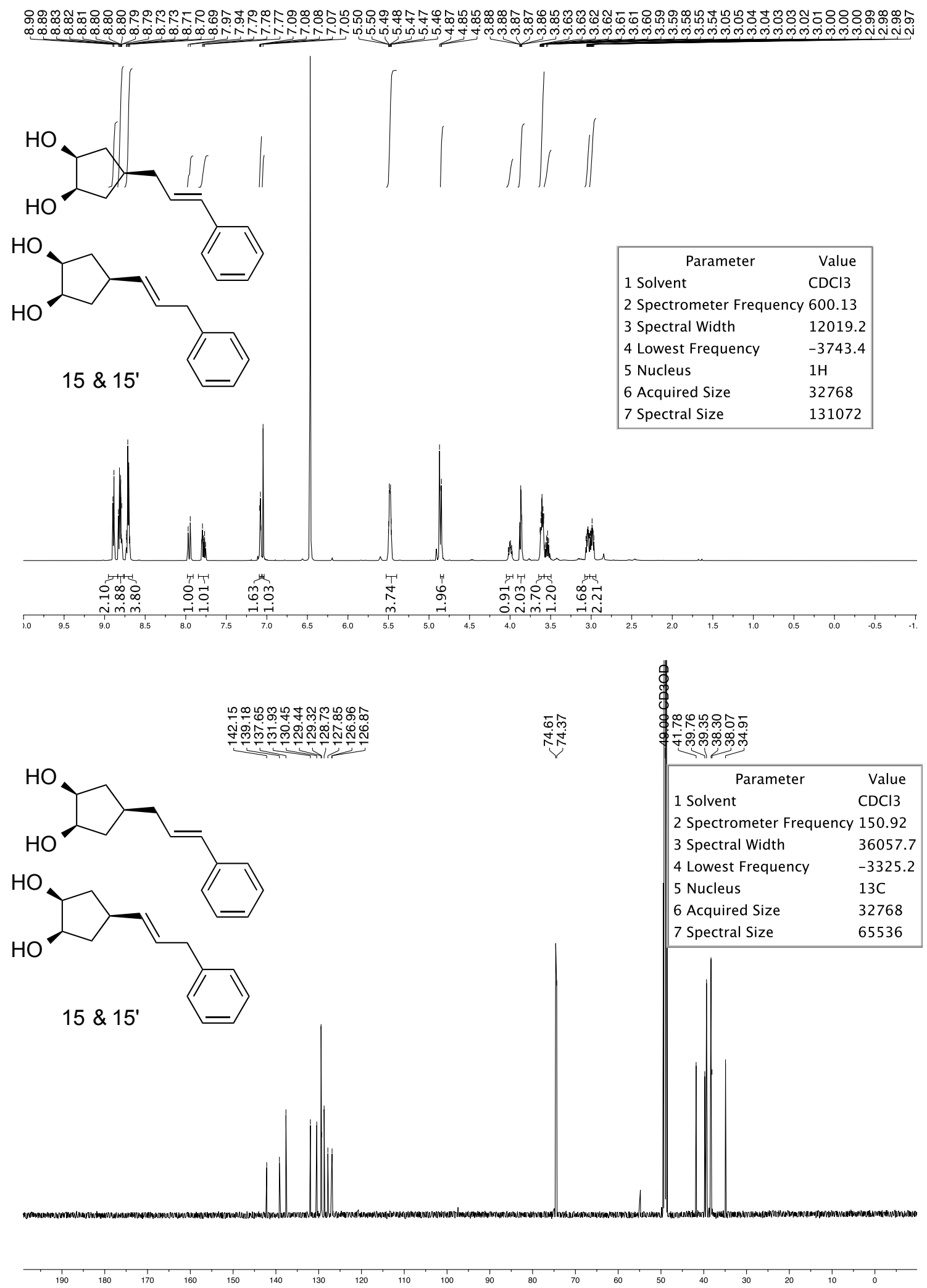


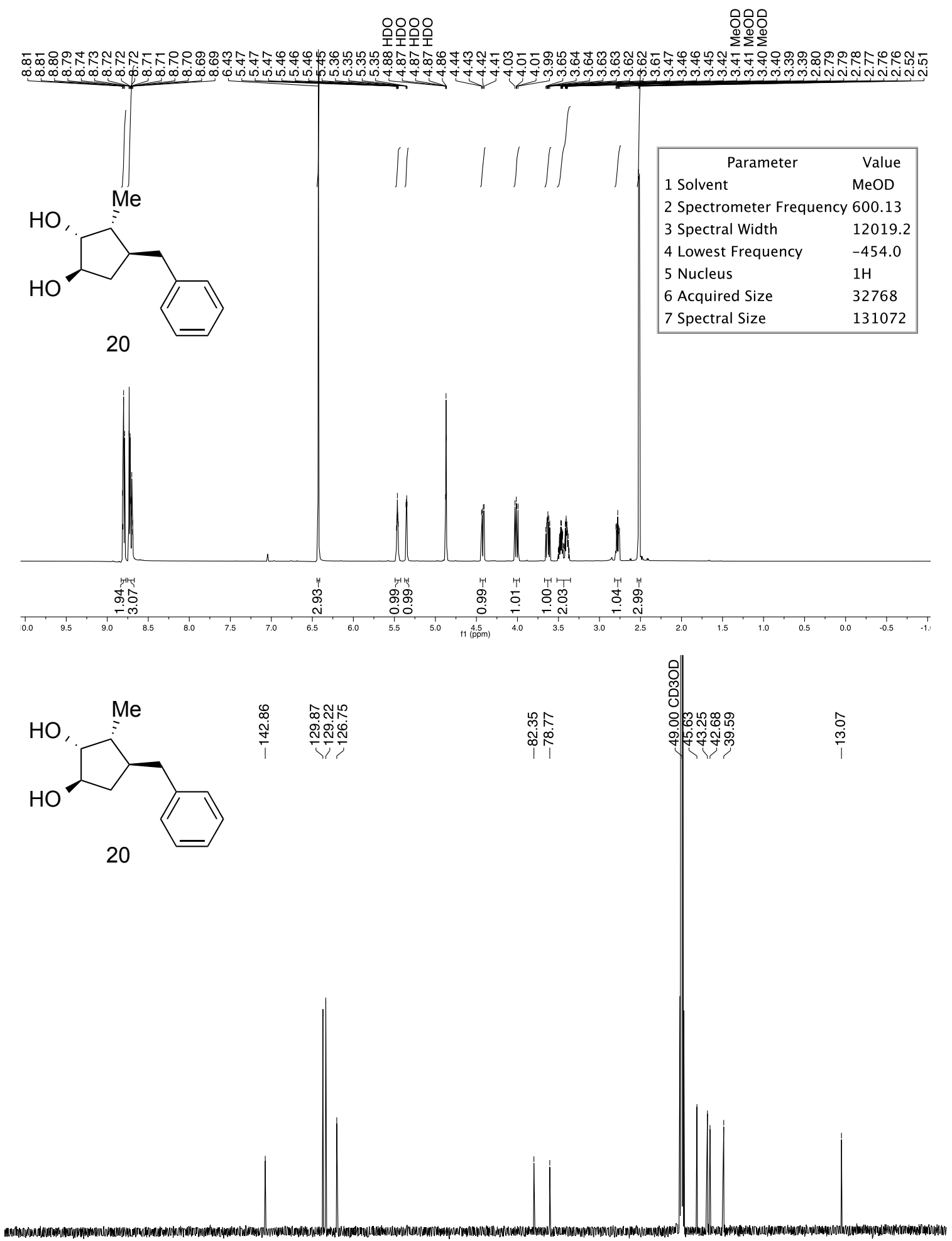

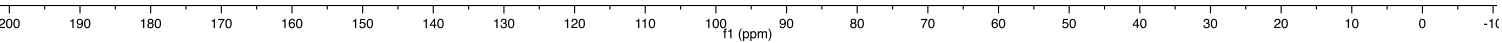



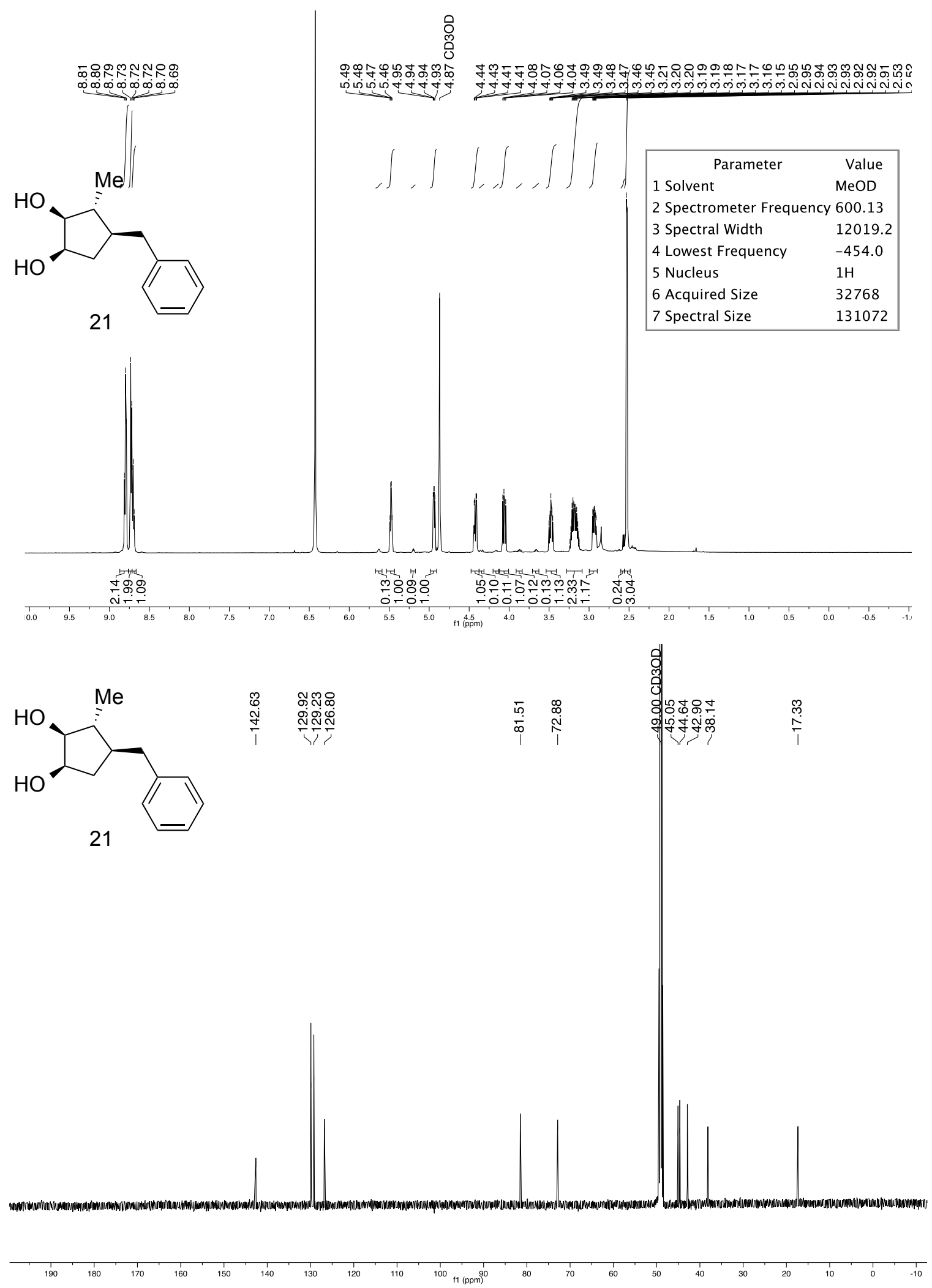

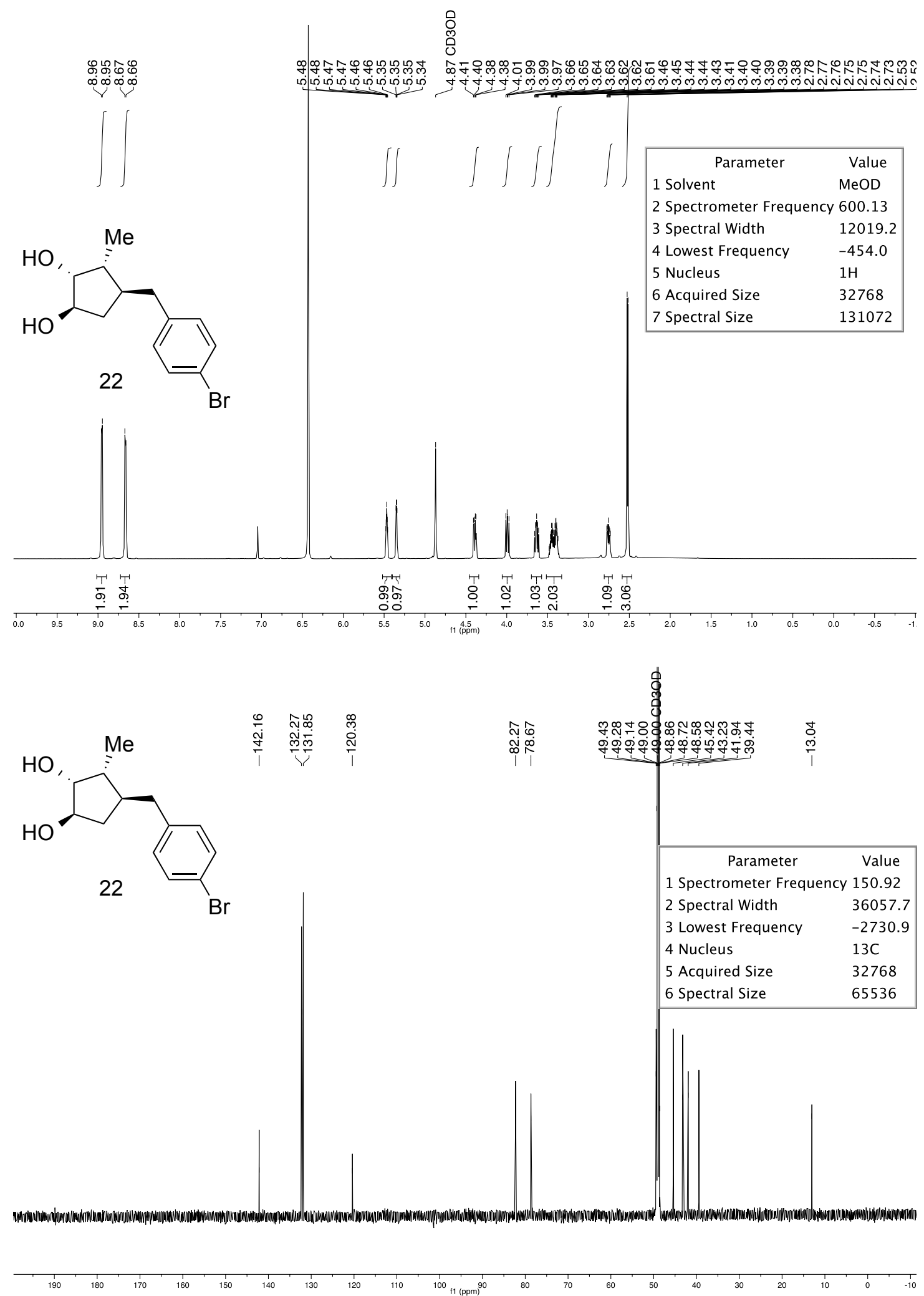

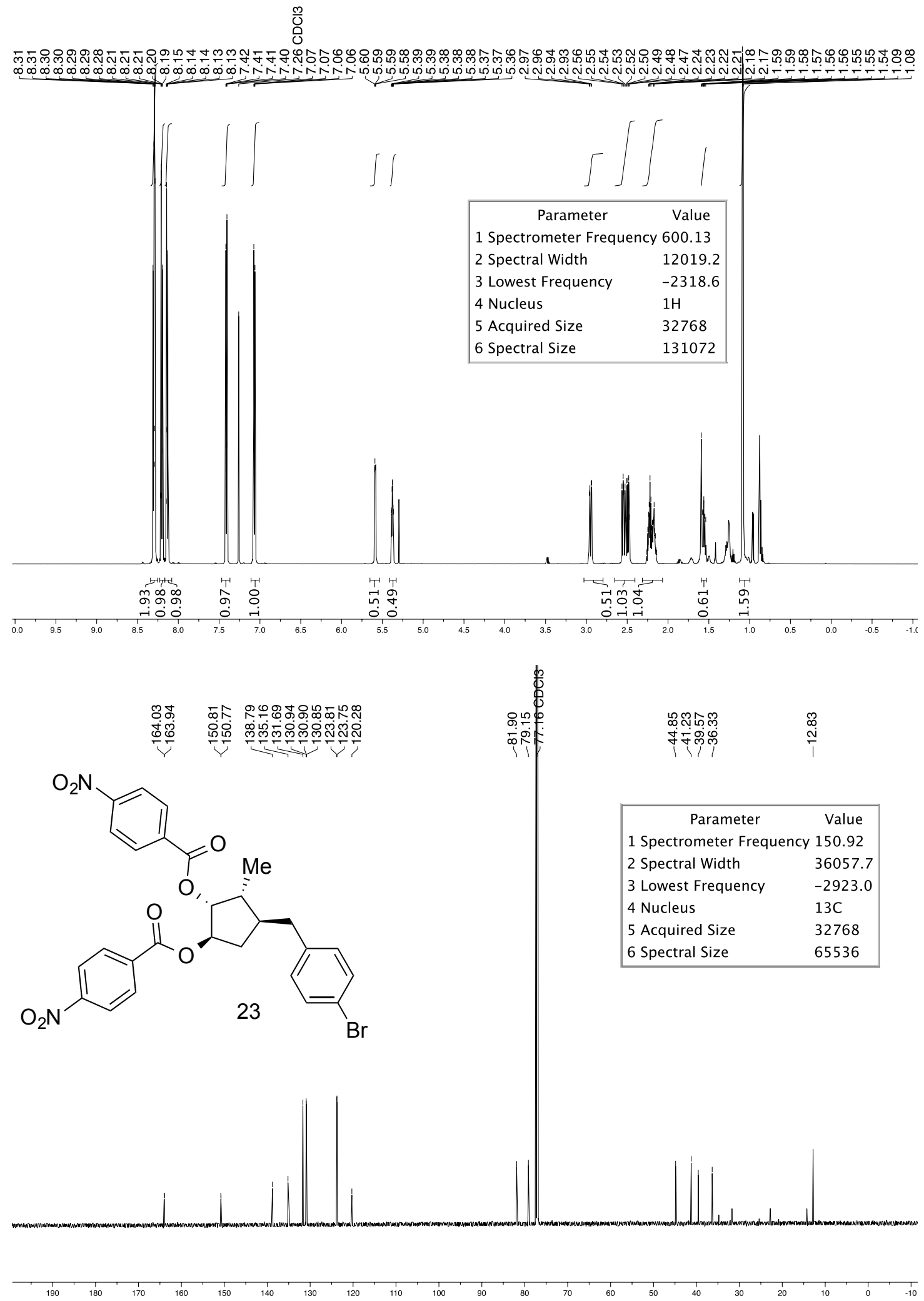

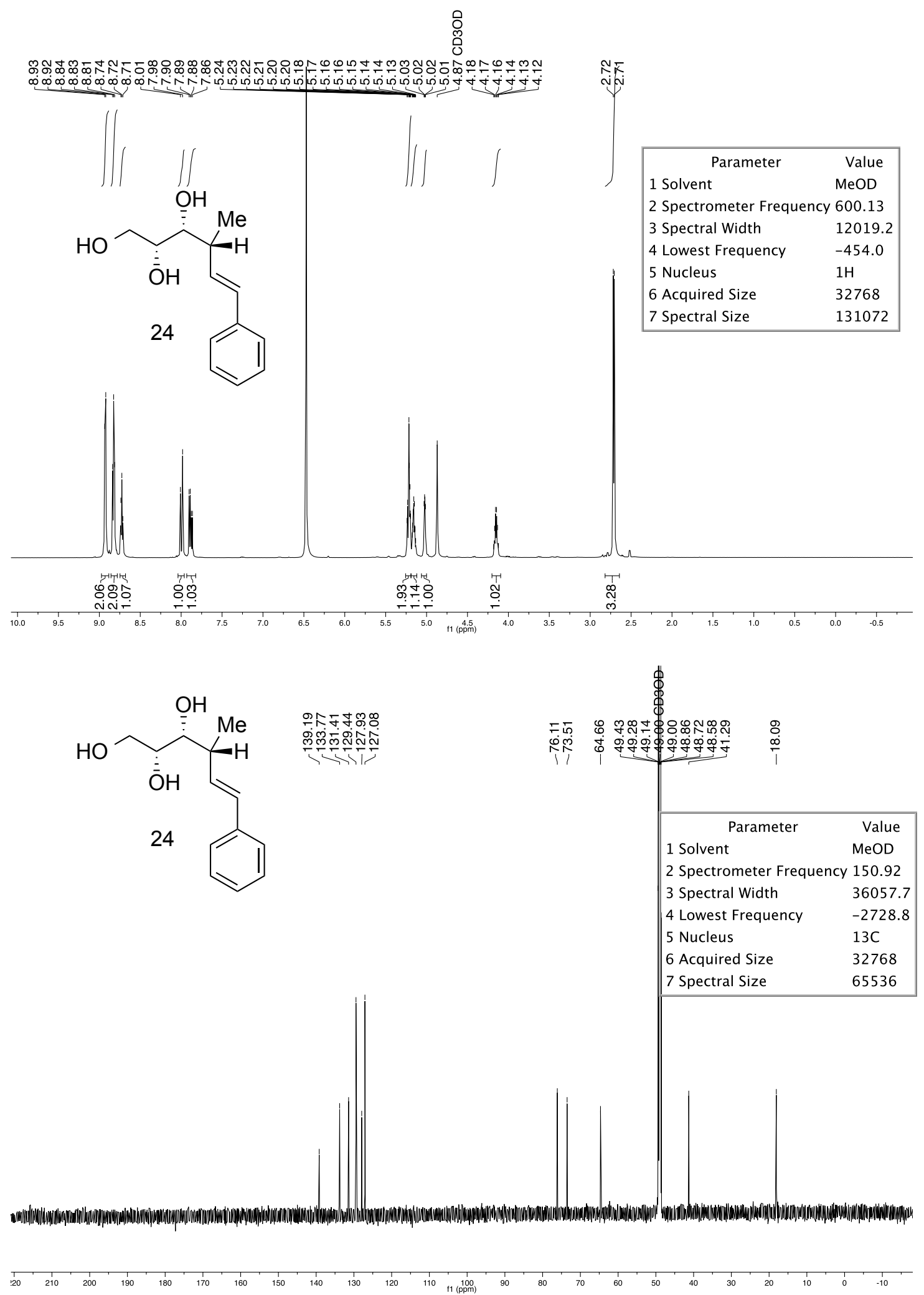

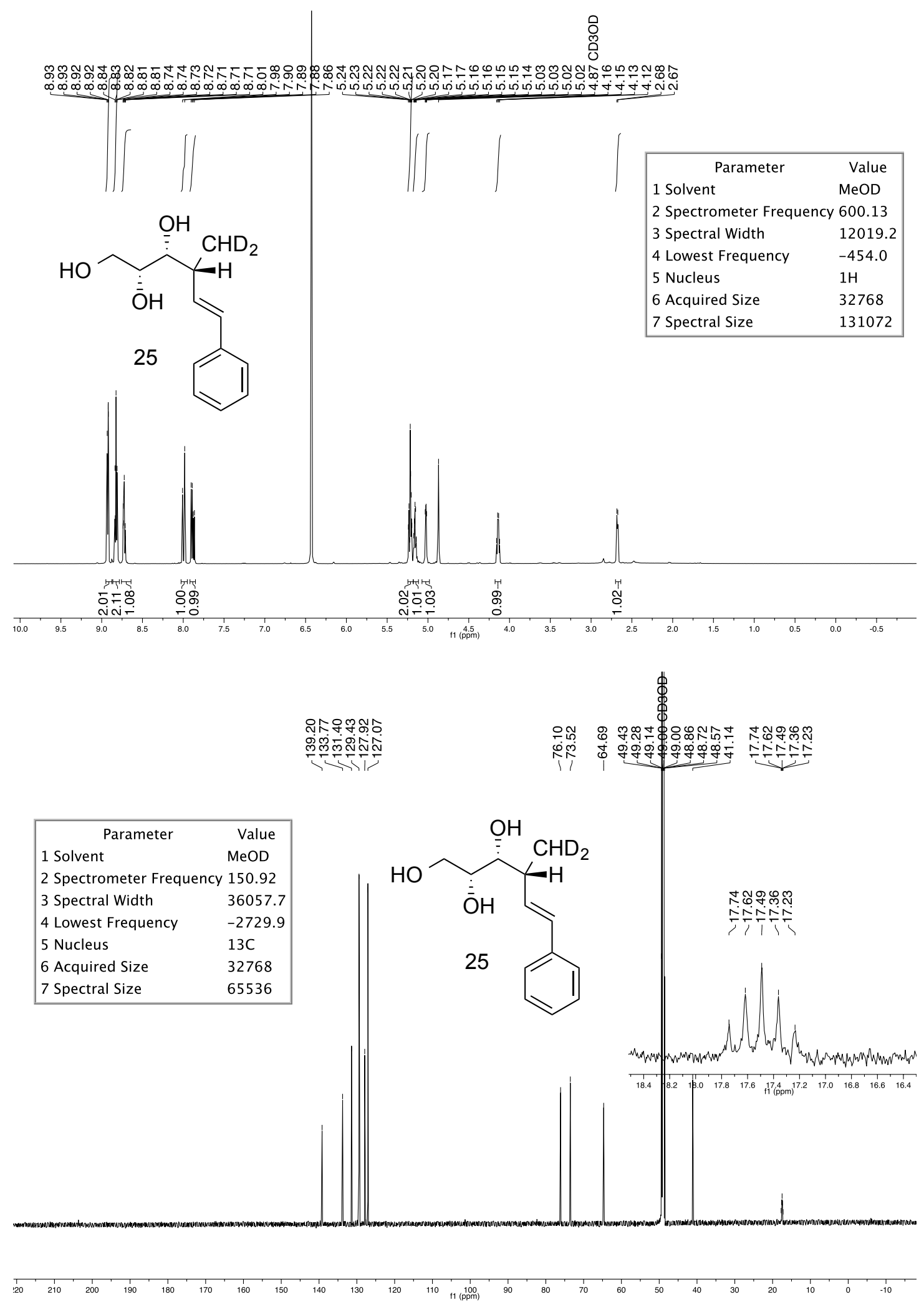

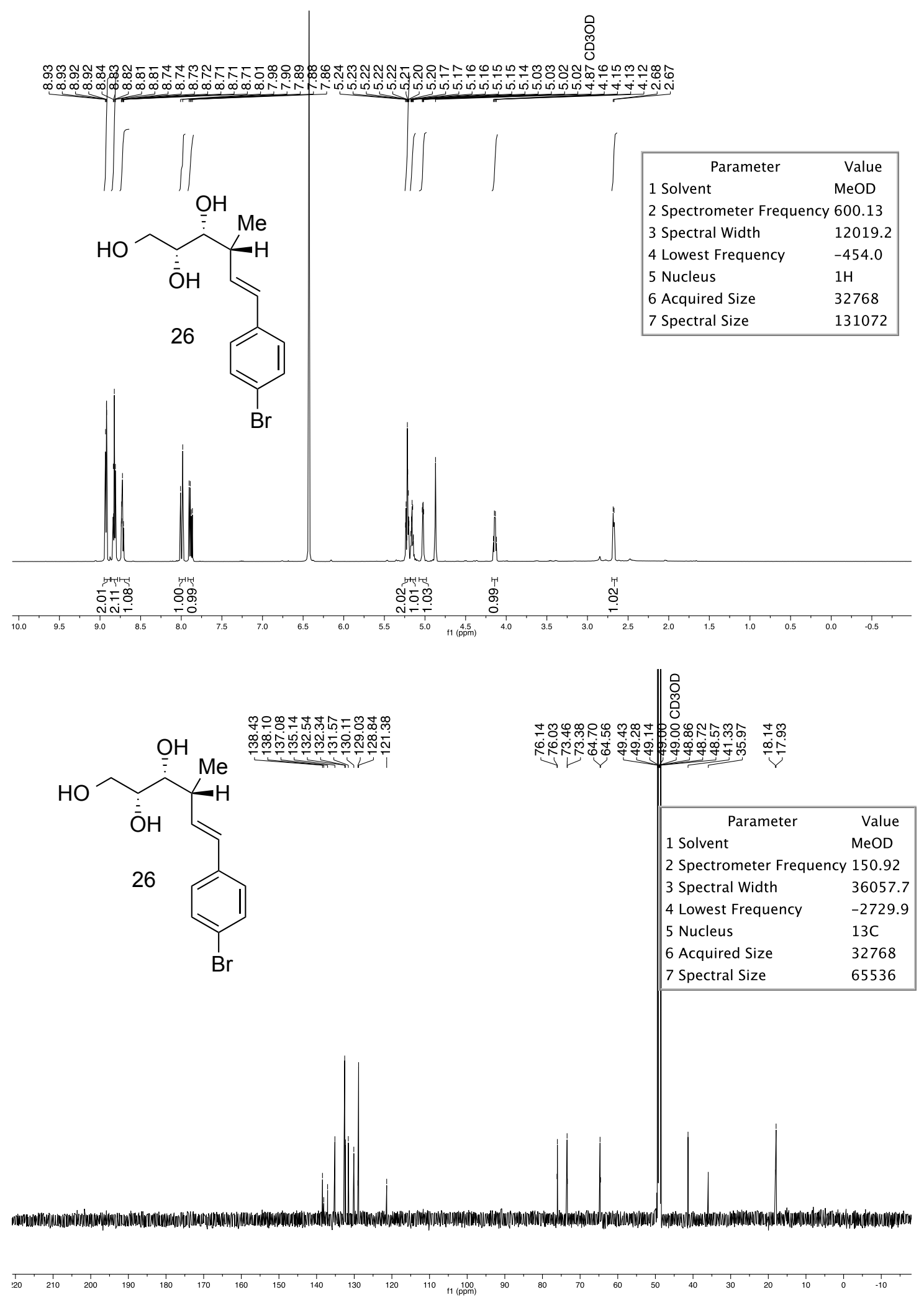

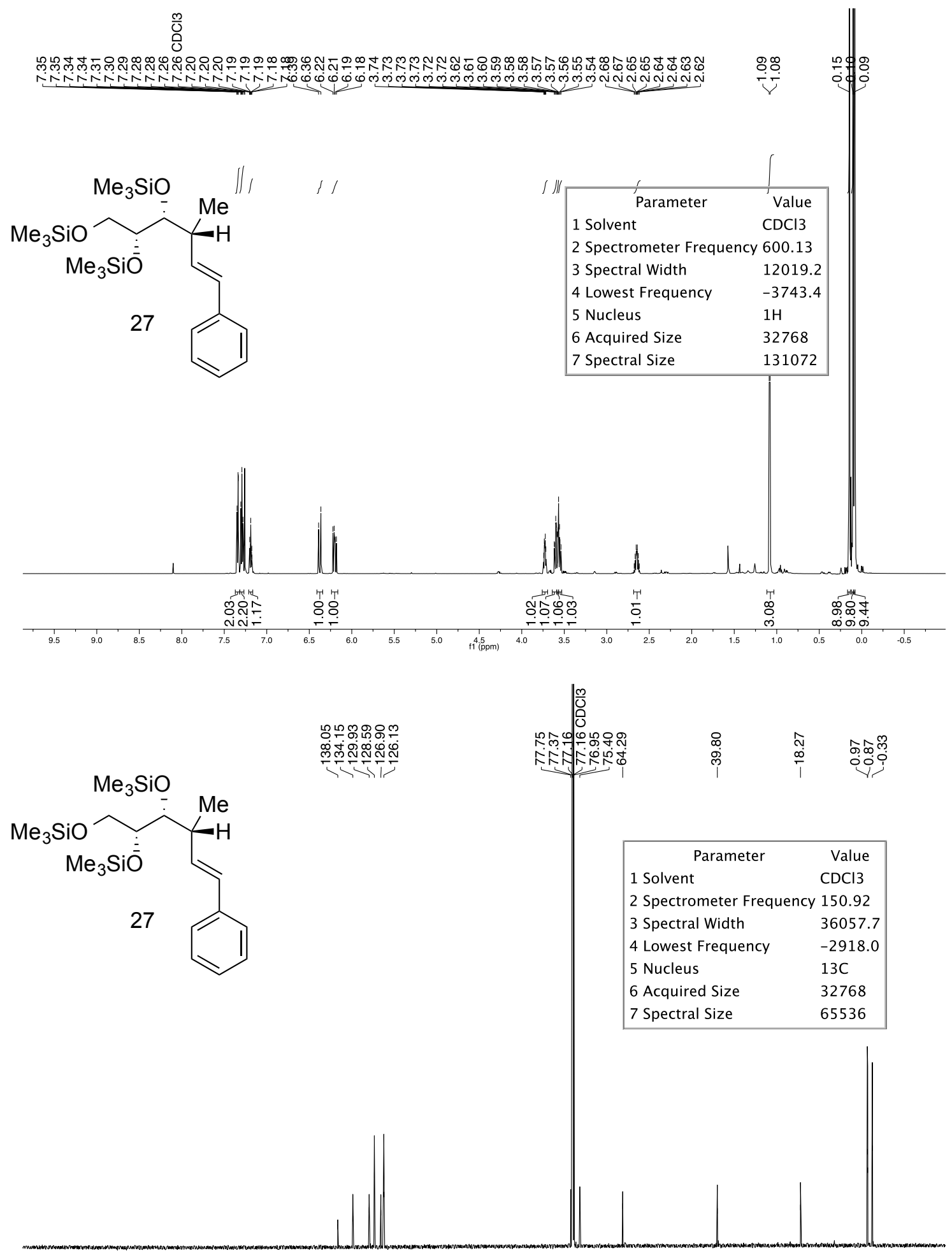

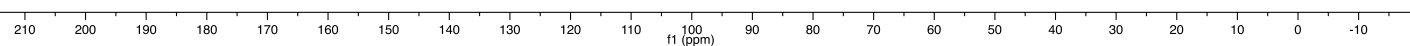



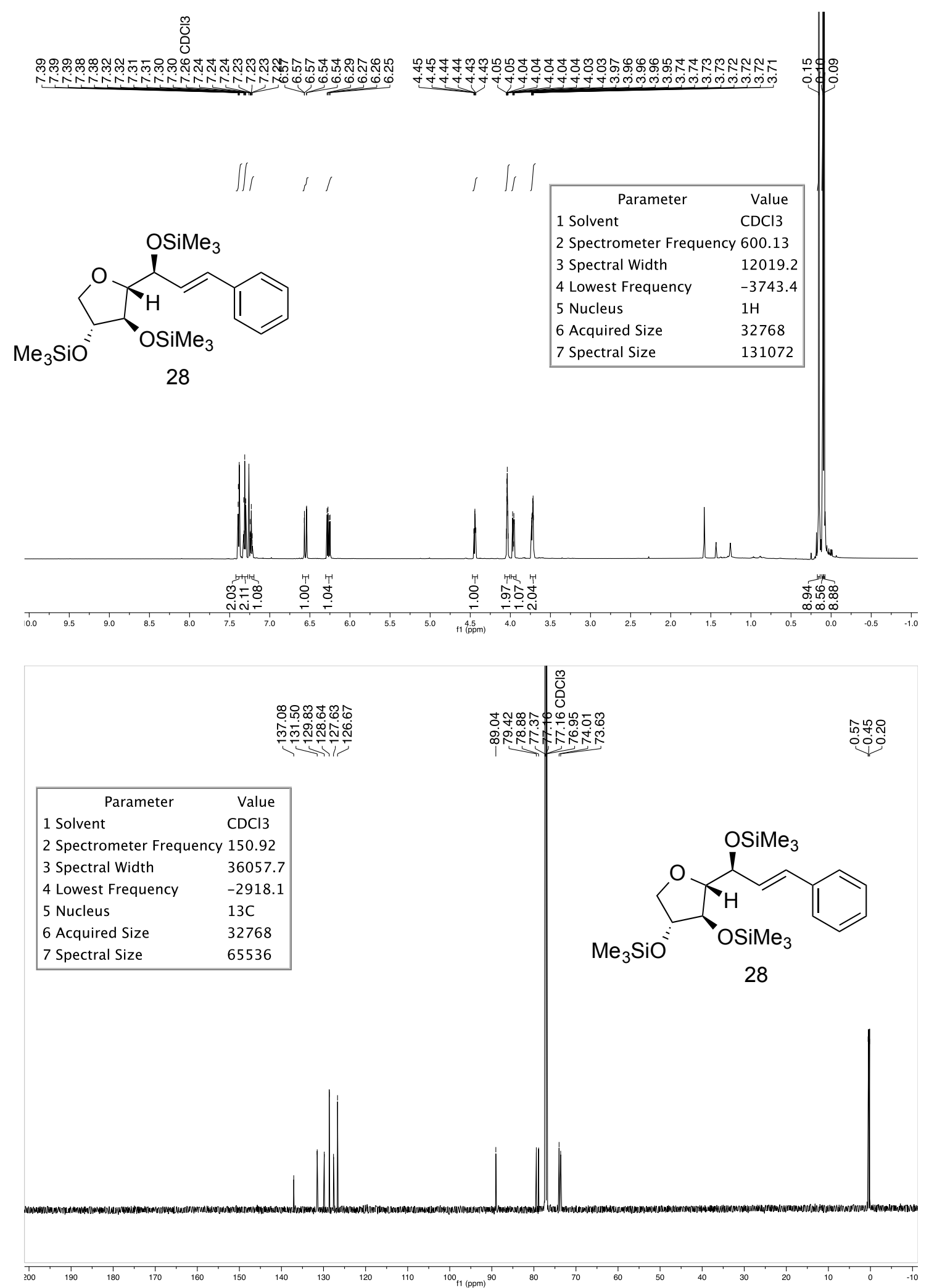

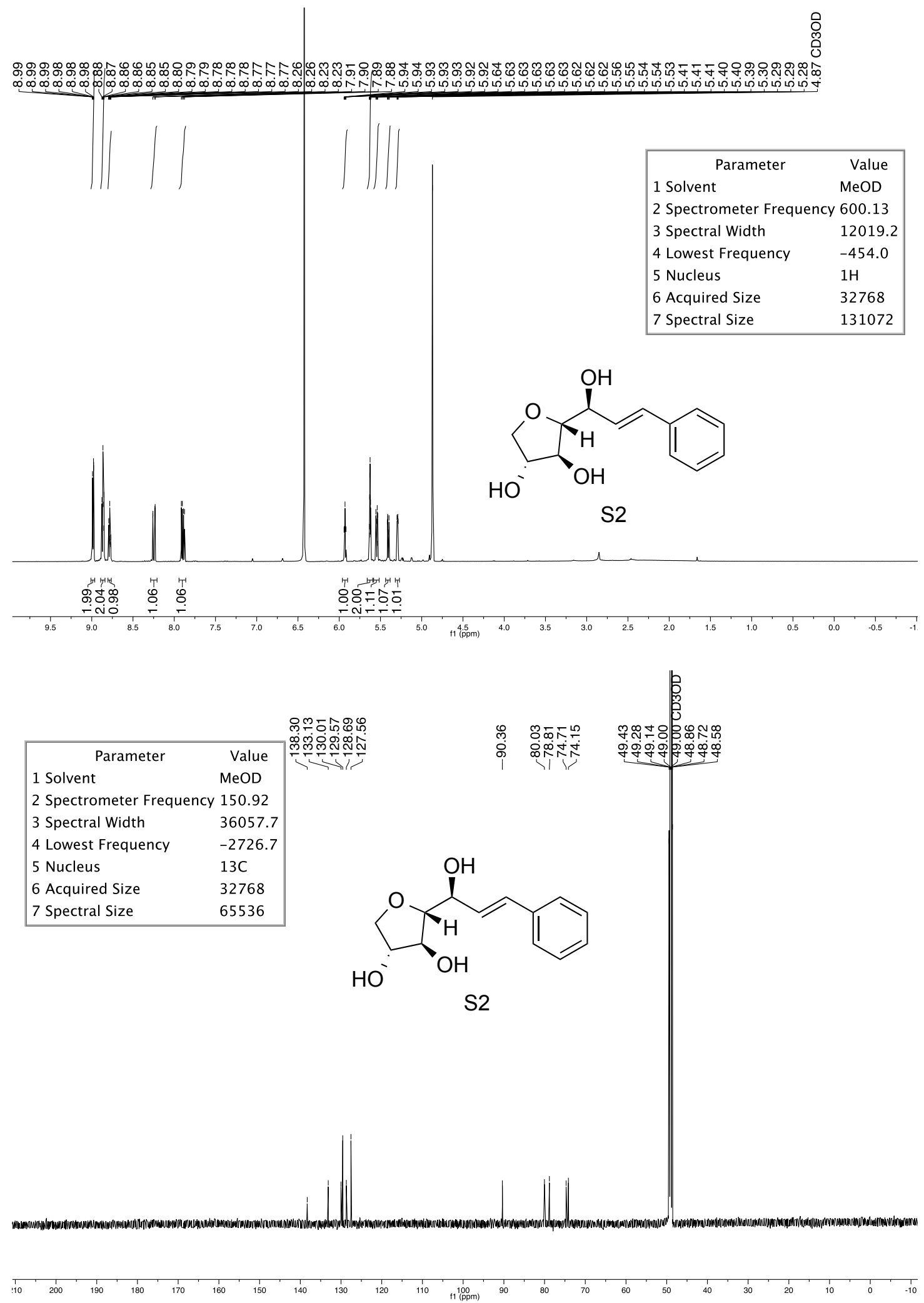